\title{
Enhanced Instantaneous Power Theory for Control of Grid Connected Voltage Sourced Converters under Unbalanced Conditions
}

\author{
Allan Alves Montanari \\ A Thesis submitted to the Faculty of Graduate Studies of the University of Manitoba \\ in partial fulfillment of the requirements of the degree of \\ Doctor of Philosophy \\ Department of Electrical and Computer Engineering \\ University of Manitoba \\ Winnipeg, Manitoba, Canada
}

Copyright $(2017$ by Allan Alves Montanari 


\section{Abstract}

This thesis introduces a new method especially designed to control the instantaneous power in voltage sourced converters operating under unbalanced conditions, including positive, negative and zero sequence content. A transformation technique, labelled mno transformation, was developed to enable the decomposition of the total instantaneous power flowing on three-phase transmission topologies into constant and oscillating terms. It is applied to three-wire and four-wire schemes, especially accommodating zero sequence unlike previous approaches.

Classical and modern electric power theories are presented, particularly focusing on their definitions for adverse $\mathrm{AC}$ scenarios. The main mathematical transformations conceived to analyze such situations are summarized, showing their respective advantages and disadvantages. An enhanced instantaneous power theory is introduced. The novel proposed power equations, named mno instantaneous power components, expands the application of the $p-q$ theory, which is attached to the $\alpha \beta 0$ transformation.

The mno instantaneous power theory is applied to develop an innovative power control method for grid connected voltage sourced converters in order to minimize power oscillations during adverse AC scenarios, particularly with zero sequence content. The method permits to sustain constant instantaneous three-phase power during unbalanced conditions by controlling independently the constant and the oscillating terms related to the instantaneous power. The effectiveness of the proposed control approach and the proposed power conditioning scheme was demonstrated using electromagnetic transient simulation of a VSC connected to an AC system. 


\section{Acknowledgements}

My immense gratitude to my wife, Juliana, for the constant encouragement along the years. Distinctive thanks to my mom and my dad, Anita and Francisco, as well as to my brother, Fernando, who have supported me through this doctorate journey. Appreciation to all my family members and friends back in Brazil who have been tuned with me while pursuing this dream.

I would like to thank my advisor Ani Gole for his guidance and support during my research program at the University of Manitoba. My appreciation to my previous advisor Cristina Tavares from University of Campinas, Brazil, during my transition to Canada. Additional thanks to Dennis Woodford and Andrew Isaacs for the period while I worked at the Electranix Corporation in Winnipeg, Canada.

I am also thankful to Remus Teodorescu and Frede Blaabjerg from Aalborg University, Denmark, where I spent part of my $\mathrm{PhD}$ program as a visiting scholar. Moreover, the early stages of this research would not be accomplished without the initial guidance of my friend Rodrigo da Silva and our innumerous discussions while in Denmark.

My gratitude for the support of my colleagues and friends in Canada who became part of my family while living in Winnipeg, as well as my coworkers and friends in Florida, USA, during the last years of this doctorate research. 
I dedicate this work to enlightened consciences, invisible to my retinas, inaudible to my eardrums, but very sensitive to my mind and my spirit through subtle channels of life. 


\section{List of Figures}

Figure 2.1: Typical three-phase transmission topologies and connections: (a) three-phase three-wire, (b) three-phase four-wire, (c) delta connection, (d) star connection .............. 9 Figure 2.2: Three-phase voltage scenarios: (a) balanced, (b) unbalanced, (c) distorted and

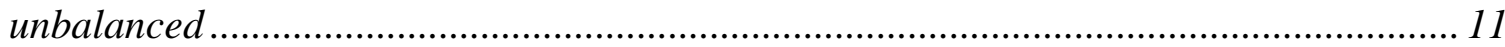

Figure 2.3: Symmetrical components of an unbalanced three-phase voltage .................. 15 Figure 2.4: The abc phasors and the $\alpha \beta$ unit vectors represented in a two-dimension

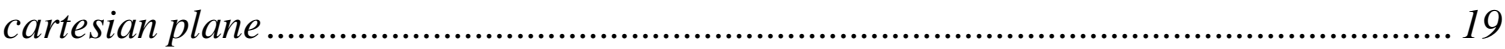

Figure 2.5: Single-phase power: (a) voltage and current, $\phi_{v}-\phi_{i}=\pi / 3$, (b) single-phase power components..... 23

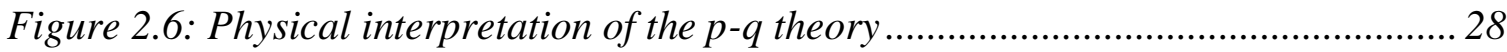

Figure 3.1: Three-dimensional abc reference frame ................................................. 37

Figure 3.2: Instantaneous normal vector: (a) orthonormal set, (b) abc normal vector

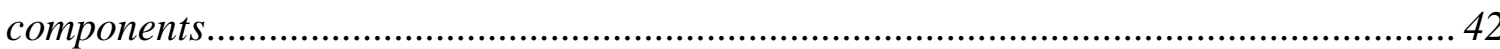

Figure 3.3: Creation of the mno basis (abc reference frame; mno reference frame; mn plane; projections; and zero sequence axis) ................................................................. 43

Figure 3.4: The mno pitch angles and the abc projections of the abc unit vectors onto the

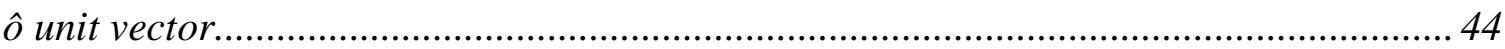
Figure 3.5: Fixed zero sequence planes: (a) view from outside the limits, (b) view from inside the limits. 46

Figure 3.6: The mno yaw angles and the abc projections of the abc unit vectors onto the

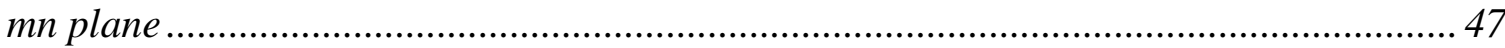

Figure 3.7: Main steps to evaluate the mno angles ...................................................... 48

Figure 3.8: Projections of the abc versors onto the $\hat{m}$ and $\hat{n}$ directions.......................... 49

Figure 3.9: Projections of the abc unit vectors onto the $\hat{m}, \hat{n}$ and $\hat{o}$ directions............. 50

Figure 3.10: Clarke's transformation represented in the abc three-dimensional coordinate system: (a) three-dimensional view, (b) view from the top of the zero sequence axis (two-

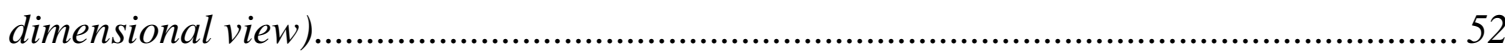


Figure 3.11: The mno and $\alpha \beta 0$ components: (a) abc voltage, (b) abc current, (c) mno voltage, (d) mno current, (e) $\alpha \beta 0$ voltage, (f) $\alpha \beta 0$ current ............................................. 56 Figure 3.12: Voltage and current signals loci in the abc and mno time domain threedimensional reference frame: (a) abc voltage and abc current, (c) mno voltage and mno

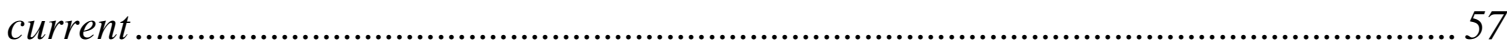

Figure 4.1: Grid connected voltage sourced converter ..............................................67

Figure 4.2: VSC bridge topologies: (a) two-level, (b) three-level diode-clamped ........... 68

Figure 4.3: Modular Multilevel Converter with half-bridge submodules ....................... 69 Figure 4.4: Two-terminal VSC configurations: (a) monopole, (b) symmetrical monopole, (c) bipole 70

Figure 4.5: Two-level voltage sourced converter zero sequence current permissive connections: (a) midpoint of DC capacitors, $(b)$ four-leg converter ............................... 72

Figure 4.6: MMC VSC with cascade H-bridge wye connected ...................................... 73

Figure 4.7: Converter connection model of the VSC to the AC network .......................... 75

Figure 4.8: The mno instantaneous power components control scheme with hysteresis

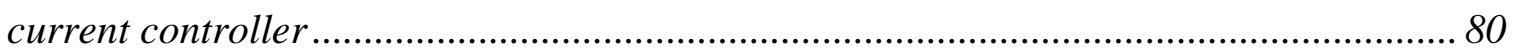

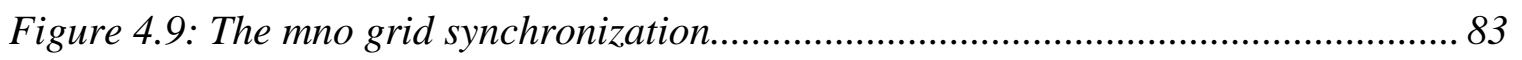

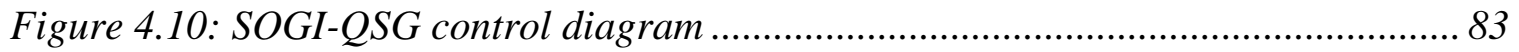

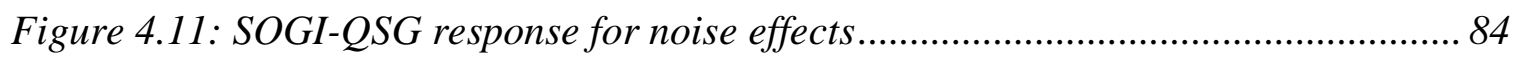

Figure 4.12: SOGI-QSG response for voltage sag and phase shift ................................85

Figure 4.13: Unbalanced voltage for Equation (4.15) and correspondent basis vector

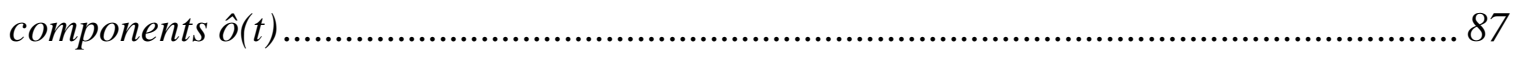

Figure 4.14: The mno angles components for Equation (4.15): mno pitch angles and mno

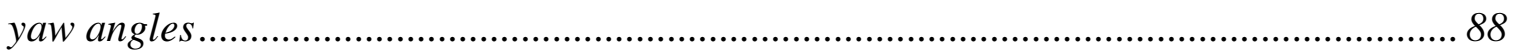

Figure 4.15: The dq mno implementation: (a) direct transformation, (b) inverse

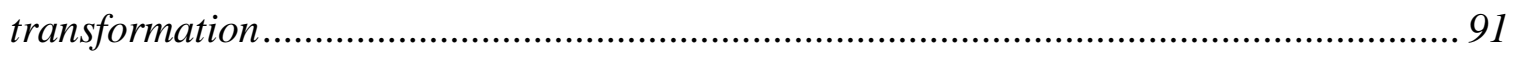

Figure 4.16: Voltage components of the mno instantaneous current components control for scenario of Equation (4.15): mno voltage components, $d q$ mno voltage components, $\alpha \beta 0$ voltage components. 94

Figure 4.17: Instantaneous power and current components of the mno instantaneous current components control for scenario of Equation (4.15): instantaneous active and 
reactive powers, dq mno current components, mno current components, abc measured

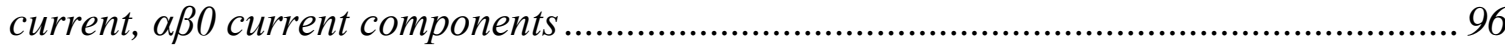

Figure 4.18: Current reference and dead band limiting signals .................................. 97

Figure 4.19: The hysteresis controlled current ........................................................... 98

Figure 4.20: The hysteresis control and the PWM pulses .......................................... 98

Figure 4.21: Voltage components and mno angles of the mno instantaneous current components control for scenario of Equation (4.21): abc measured voltage, mno pitch angles, mno yaw angles, mno voltage components, $d q$ mno voltage components, $\alpha \beta 0$ voltage components 100

Figure 4.22: Instantaneous power and current components of the mno instantaneous current components control for scenario of Equation (4.21): instantaneous active and reactive powers, dq mno current components, mno current components, abc measured current, current components 101

Figure 4.23: Voltage components and mno angles of the mno instantaneous current components control for scenario of Equation (4.22): abc measured voltage, mno pitch angles, mno yaw angles, mno voltage components, $d q$ mno voltage components, $\alpha \beta 0$ voltage components. 102

Figure 4.24: Instantaneous power and current components of the mno instantaneous current components control for scenario of Equation (4.22): instantaneous active and reactive powers, dq mno current components, mno current components, abc measured current, $\alpha \beta 0$ current components ..... 103 Figure 4.25: Voltage components and mno angles of the mno instantaneous current components control for scenario of Equation (4.23): abc measured voltage, mno pitch angles, mno yaw angles, mno voltage components, $d q$ mno voltage components, $\alpha \beta 0$ voltage components 105

Figure 4.26: Instantaneous power and current components of the mno instantaneous current components control for scenario of Equation (4.23): instantaneous active and reactive powers, dq mno current components, mno current components, abc measured current, $\alpha \beta 0$ current components 106 
viii

\section{List of Tables}

Table 4.1: Grid synchronization values of the unit basis vector $\hat{o}$ and mno angles for balanced voltage condition at $400 \mathrm{~ms}$ and unbalanced voltage condition of Equation (4.15) at $500 \mathrm{~ms}$ 


\section{Abbreviations}

$\mathrm{AC}-$ Alternate Current

DC - Direct Current

FACTS - Flexible Alternating Current Transmission System

HCC - Hysteresis Current Control

HVDC - High Voltage Direct Current

IGBT - Insulated-Gate Bipolar Transistor

MMC - Modular Multilevel Converter

PCC - Point of Common Coupling

PI - Proportional Integral

PWM - Pulse Width Modulation

SOGI - Second Order Generalized Integrator

SPWM - Sinusoidal Pulse Width Modulation

STATCOM - Static Synchronous Compensator

QSG - Quadrature Signal Generator

VSC - Voltage Sourced Converter 


\section{Table of Contents}

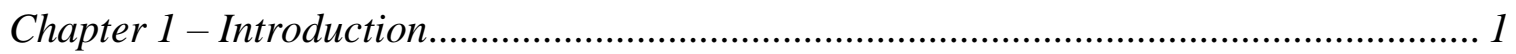

1.1 - Evolution of AC and DC Transmission Networks ............................................ 2

1.2 - Adverse Power Transmission Scenarios........................................................... 4

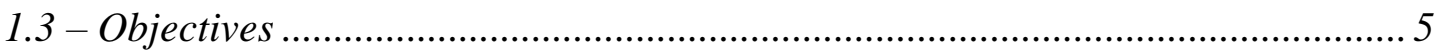

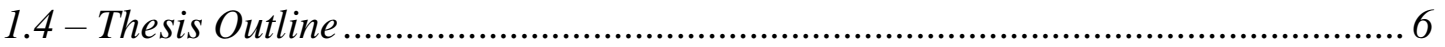

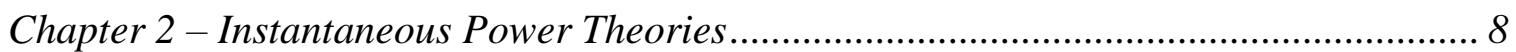

2.1 - Three-Phase Transmission Topologies and Adverse Operational Conditions ... 8

2.2 - Vector Transformations .............................................................................. 11

2.2.1 - The Symmetrical Components in the Frequency Domain........................ 12

2.2.2 - The Symmetrical Components in the Time Domain................................. 15

2.2 .3 - The $\alpha \beta 0$ Transformation ……………………........................................ 16

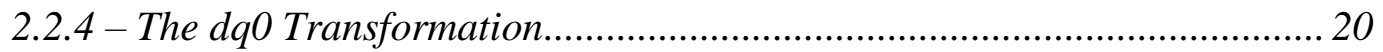

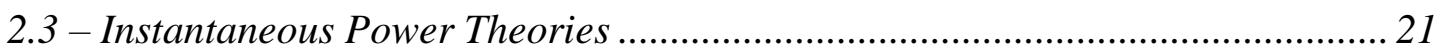

2.3.1 - Classical Power Definitions.................................................................. 22

2.3.2 - Power Concepts under Adverse Conditions............................................. 24

2.4 - The p-q Instantaneous Power Theory.............................................................. 29

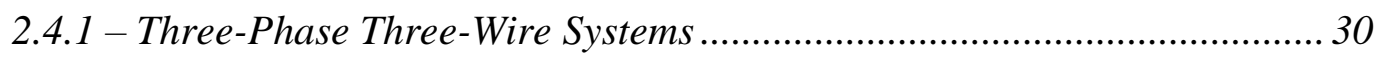

2.4.2 - Three-Phase Four-Wire Systems …………………………………......... 31

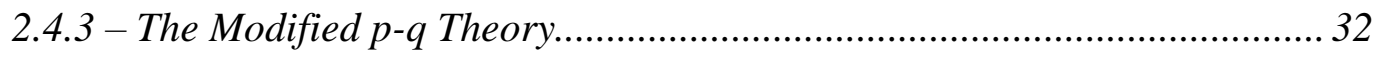

Chapter 3 - The mno Instantaneous Power Theory....................................................... 35

3.1 - The Three-Dimensional Time Domain Reference Frame.................................. 35

3.2 - The mno Transformation ................................................................................ 38

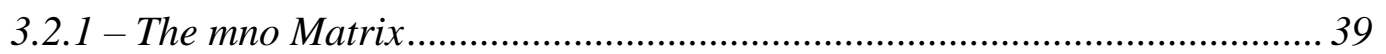

3.2.2 - The Instantaneous Normal Vector ......................................................... 40

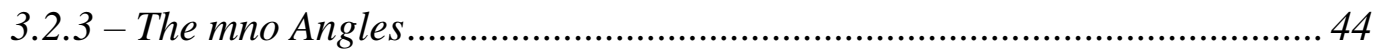

3.2.4 - The $\alpha \beta 0$ Transformation as a Special Case of the mno Transformation . 50

3.2.5 - The Direct and Quadrature mno Matrix.................................................. 53

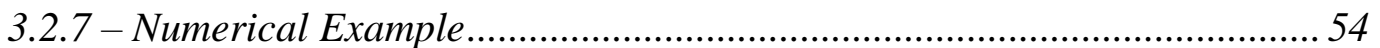

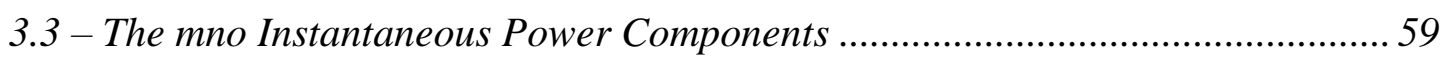


Chapter 4 - The mno Instantaneous Power Components Control for Grid Connected

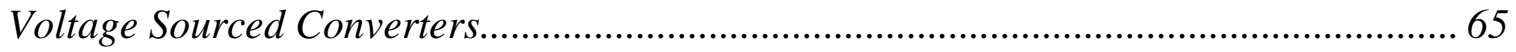

4.1 - Grid Connected Voltage Sourced Converters ..................................................6 66

4.1.1 - Converter Bridge Topologies and AC/DC Interface Equipment's

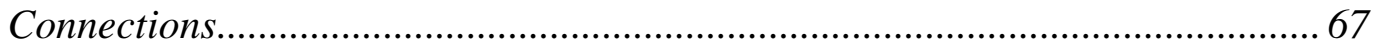

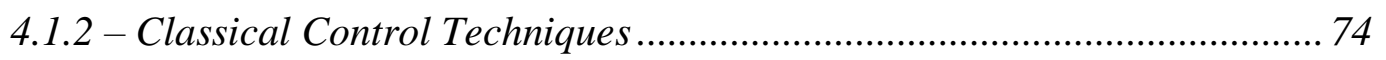

4.1.3 - Control Methods under Adverse AC Conditions..................................... 77

4.2 - The mno Instantaneous Power Components Control ...................................... 79

4.2.1 - The Grid Connected Voltage Sourced Converter Test System ................ 81

4.2 .2 - Grid Synchronization ................................................................... 82

4.2.3 - The dq mno and the Inverse dq mno Implementation ............................. 89

4.2.4 - Power Control Strategies...................................................................... 91

4.2.5 - The Hysteresis Current Controller Implementation .............................. 97

4.2.6 - Unbalanced and Balanced Application Scenarios................................ 99

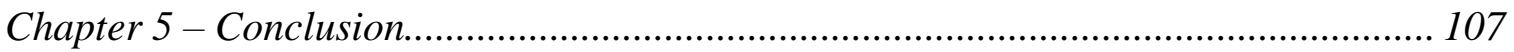

5.1 - Main Contributions and Conclusions .......................................................... 107

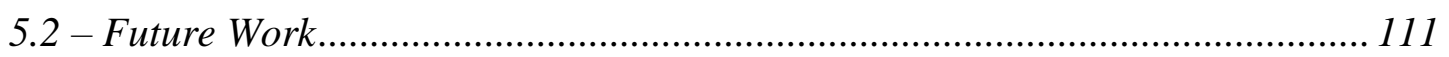

5.2.1 - Synchronous mno Transformation ................................................... 111

5.2.2 - Reactive Power Control Strategies ................................................... 112

5.2.3 - Control Improvements and Performance Evaluation ........................... 112

5.2.4 - VSC Application Cases ................................................................ 113

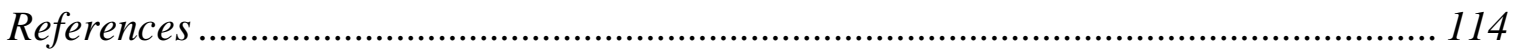




\section{Chapter 1}

\section{Introduction}

"They see only the shadows which the fire throws on the opposite wall of the cave? How could they see anything but the shadows if they were never allowed to move their heads?" Paraphrasing the sophisms of Socrates from the Allegory of the Cave [1], it is essential to conceptualize models with accurate number of dimensions, such as in a threedimensional space, rather than seeing ordinary shadows, for instance in a two-dimensional space. Likewise, this work humbly aims to solve a challenging engineering problem by launching a new visualization method to devise it.

The problem appears when pondering upon some questions related to power transmission: How is the instantaneous power phenomena described for general AC conditions? Is there an advantageous manner to instantaneously calculate the power transfer during unbalanced scenarios? Is it possible to manage grid connected voltage sourced converters to fully control the instantaneous power when the system is unbalanced?

In the course of addressing these topics, simple, but powerful techniques are proposed throughout this work. The introduction of new methods provide a step closer to 
answer the prior questions, expanding the related engineering knowledge towards the solution of the problem which will be exposed.

\section{1 - Evolution of AC and DC Transmission}

\section{Networks}

Since the late 1880 s, bulk electric energy generation has become a reality, fulfilling primarily illumination purposes, as well as production of motion and heat. The transmission system was born to allow the power delivery between generation stations and customers, combining electric current and electric potential on conductor mediums.

Two types of transmission systems came into view during the power systems early times, competing to each other in the War of Currents era [2]: the alternating current (AC) and the direct current (DC). The AC transmission quickly became feasible and took over the DC system, mainly due to the invention of the power transformer [3], conveying electrical energy over long distances.

The three-phase AC transmission system spread out on the following decades, being far more economical in the early times, establishing itself as an efficient power transmission scheme. Such technology expanded into complex meshed networks, demanding the development of numerous auxiliary apparatus and control methods to guarantee a consistent controlled power delivery through the entire grid.

Although DC transmission concepts were previously known, the technology turned to be suitable for high voltage transmission only after the middle of the twentieth century. Many years of continued work were demanded to achieve the development into 
commercial systems. The DC revival was driven by the development of high voltage mercury arc valves [4], which were unavailable during the War of Currents. These mercury valves were used in DC systems designed worldwide since its first project in 1954 up to the 1970s. The Manitoba Nelson River Bipole I (Manitoba, Canada) was the last system in the world commissioned using such technology [5].

After the 1970s, semiconductor devices propelled an increasing deployment of DC transmission systems. Many projects were implemented using thyristors, replacing the mercury arc technology, and the world's first thyristor multiterminal DC system was commissioned between Quebec (Canada) and New England (USA).

In the late 1990s, the penetration of voltage sourced converters DC systems into electric networks was nurtured by the development of the higher rated self-commutated transistors [6]. And more recently, DC grids using voltage sourced converters emerged as an elegant solution to trade energy across borders, integrating more than two locations over long distances with DC networks [7].

These different technologies are part of the modern networks, with AC and DC branches, interconnected by power converters in a hybrid intricate system $[8,9]$. The alternate and direct current systems must share their advantages and disadvantages, jointly improving the overall power flow controllability of the grid. They should cooperate to each other mainly while subjected to challenging adverse operational conditions, which have demanded advanced research and development efforts to explore innovative modes of operation in order to deliver the electric power. 


\section{2 - Adverse Power Transmission Scenarios}

The power converters, main equipment at the interface between the AC and DC systems, are able to enhance the power transfer capability by controlling certain electric variables of the transmission lines where they are connected. In particular, voltage sourced converters have been employed to fulfill many applications related to supply of power to weak or passive networks, renewable energy integration, grid stability support and power quality improvement [10-12], among others.

However, different types of contingencies may impose crucial challenges for the operation of the voltage sourced converters. Asymmetrical transient faults or unbalanced loads, for example, force these devices to operate under unbalanced conditions (three-phase voltage and current with different magnitudes or different shift angles among the phases). This adverse scenario may lead to severe power oscillations through the AC/DC interface, depending on the characteristics of the dynamic transients and the control systems available at the power converter.

The voltage sourced converters are ideally expected to sustain the power orders even during these grid disturbances, riding through as many transient events as possible according to different grid codes. They should attend the increasing demands related to low-voltage-ride-through capability, with minimized impact on the power transfer $[13,14]$.

Many technical solutions have been proposed to deal with voltage sourced converters operating under unbalanced and distorted voltage conditions [15-20], for example by injecting appropriately synthesized unbalanced currents. Nevertheless, dealing with zero sequence transients is still complicated, as it will be explained in the thesis. The oscillating zero sequence power [65] affects the total three-phase energy flow per time unit, 
causing power oscillations. This is one of the reasons why zero sequence content is not welcome in most circuits, being frequently avoided [65].

The approach proposed and described in this thesis allows a constant power transmission to be sustained under unbalanced AC scenarios, even in the presence of zero sequence voltage and zero sequence current.

\section{3 - Objectives}

The present work introduces control methods especially designed to deal with positive, negative and zero sequence power through grid connected voltage sourced converters. The motivation emerged due to the lack of robust controllers to handle power oscillations during unbalanced conditions characterized by positive, negative and zero sequence content. The proposed control method allows three-phase instantaneous constant power transmission even in the presence of zero sequence voltage and zero sequence current components.

The pursuit of improved power control methods triggered investigations related to the calculation itself of the instantaneous power during unbalanced operation. Unfortunately, classical power theory definitions developed for three-phase transmission systems are not suitable to analyze unbalanced or distorted voltages and currents waveforms. Thus, investigations on the instantaneous power phenomena and calculation methods become vital to determine the reference currents by the control system of the power converters and, therefore, explore new power control techniques for voltage sourced converters. 
In the light of the numerous power theories which have been developed, this thesis explores the related state-of-the-art. It then introduces an innovative transformation tool that provides a distinct method to visualize and calculate the instantaneous power on threephase transmission lines, exposing its advantages during unbalanced circumstances.

Furthermore, fulfilling the engineering nature of this work, this technique is applied on the design of control strategies for power conditioning devices, presenting a control scheme that enhances the power transfer capability through VSCs, mitigating power oscillations. The effectiveness of the proposed control approach and the proposed power conditioning scheme was demonstrated using electromagnetic transient simulation of a voltage sourced converter connected to an AC system.

\section{4 - Thesis Outline}

After this introductory chapter, the remaining structure of this document is as follows:

Chapter 2 reviews the vector transformations and definitions behind electric power theories developed for the analysis of adverse AC scenarios, presenting the $p$ - $q$ theory, which sets the stage for the development of the mno power theory.

Subsequently, Chapter 3 introduces the mno power theory, describing details of the $m n o$ vector transformation and the mno instantaneous power components, which allow to decompose the total instantaneous power calculation into constant and oscillating terms for general three-phase transmission topologies under either balanced or unbalanced conditions. 
Chapter 4 presents the main application that motivated the development of the mno instantaneous power components: a new method able to separately control the constant and the oscillating terms of the total instantaneous power flowing through voltage source converters. Such scheme allows the power orders related to the oscillating terms to be set to zero, ordering the desired instantaneous active and reactive power through the constant terms, thus sustaining a constant power flow under challenging scenarios.

Finally, Chapter 5 summarizes the main contributions and conclusions of the thesis. 


\section{Chapter 2}

\section{Instantaneous Power Theories}

This chapter presents a background in regards to electric power theories developed for general three-phase transmission systems, particularly focusing on unbalanced and distorted conditions. The main mathematical transformations conceived to analyze such situations are summarized, culminating with the introduction of the $p$ - $q$ theory, which sets the scene for the development of the proposed mno theory (Chapter 3).

\section{1 - Three-Phase Transmission Topologies and}

\section{Adverse Operational Conditions}

Three-phase systems are broadly employed worldwide in alternating current networks for generation, transmission and distribution of electrical energy. In such systems, each phase is fed with an alternating voltage and carries an alternating current, providing means for the transmission of electric power.

These three-phase transmission networks may be designed as three-wire (separated conductors for each of the three phases) or four-wire systems (separated conductors for each of the three phases plus the neutral conductor). In high voltage transmission, three- 
wire schemes are preferred. Alternatively, the configuration with the neutral link is commonly adopted in distribution systems where imbalance is more prevalent because of differing loads. In between the transmission line sections, other equipment connected to the network (generators, transformers, loads, etc) are typically arranged in delta or wye $[22,23]$. Figure 2.1 illustrates these layouts and connections.

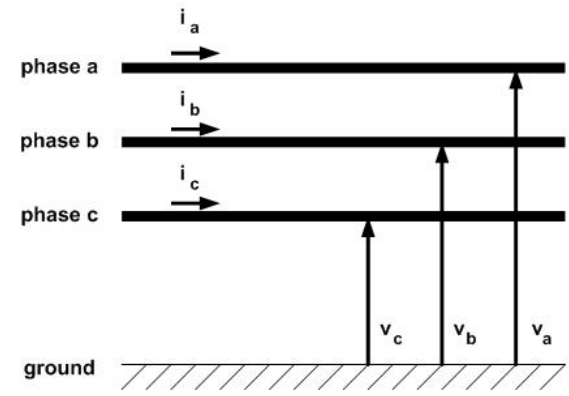

(a)

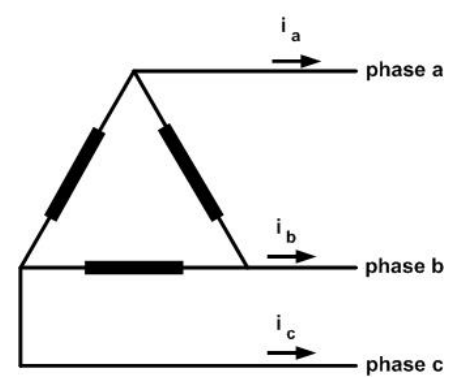

(c)

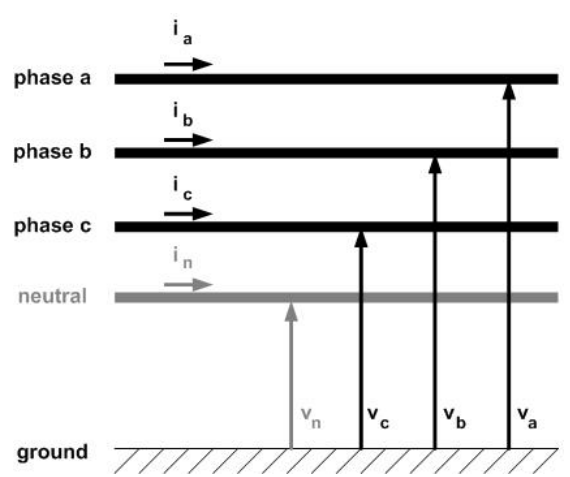

(b)

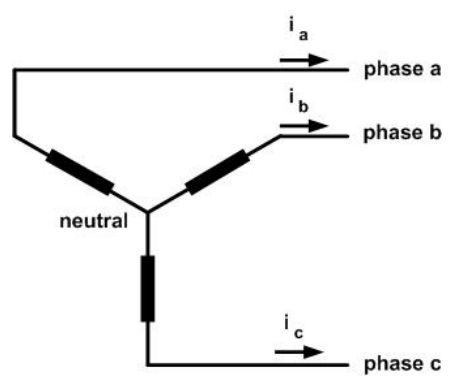

(d)

Figure 2.1: Typical three-phase transmission topologies and connections: (a) three-phase three-wire,

(b) three-phase four-wire, (c) delta connection, (d) star connection

The ground (or earth) is the reference from which the phase voltages are measured. The common arrangements between the ground and the neutral can be summarized as: ungrounded (isolated or floating neutral); solid grounded (short-circuit); resistance grounded; and reactance grounded (highly reactive impedance, e.g. zig-zag transformers) $[24,25]$. 
The connection between the ground and the neutral, along with detailed diagrams about electric equipment and the transmission lines connections are essential to evaluate how the electric power is being instantaneously transferred through the network, especially during transients, when the system is not balanced.

The three-phase system is considered to be balanced when the three-phase voltage and current signals are sinusoidal waveforms with same frequency, same magnitude, and phase shift of one-third of the cycle among each phase $(2 \pi / 3 \mathrm{rad})$. The set of balanced voltages and currents can be mathematically and respectively expressed as:

$$
\begin{aligned}
& \left\{\begin{array}{l}
v_{a}(t)=V \cos \left(\omega t+\phi_{v}\right) \\
v_{b}(t)=V \cos \left(\omega t+\phi_{v}-2 \pi / 3\right) \\
v_{c}(t)=V \cos \left(\omega t+\phi_{v}+2 \pi / 3\right)
\end{array}\right. \\
& \left\{\begin{array}{l}
i_{a}(t)=I \cos \left(\omega t+\phi_{i}\right) \\
i_{b}(t)=I \cos \left(\omega t+\phi_{i}-2 \pi / 3\right) \\
i_{c}(t)=I \cos \left(\omega t+\phi_{i}+2 \pi / 3\right)
\end{array}\right.
\end{aligned}
$$

where $V$ and $I$ refer to the peak voltage and peak current magnitudes, respectively; $\omega$ corresponds to the angular frequency; $\phi_{v}$ and $\phi_{i}$ refer to the voltage and current phase angle related to a common reference.

The balanced condition is the expected operational state of the power system. However, the system may operate under adverse conditions, categorized by unbalanced and distorted phenomena (Figure 2.2). 


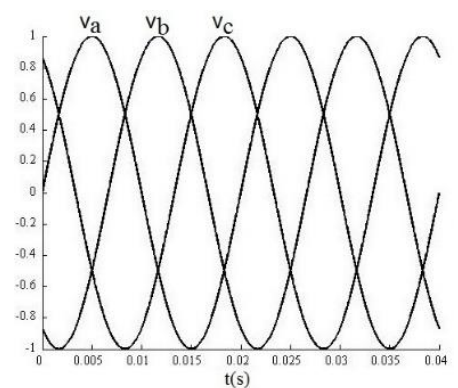

(a)

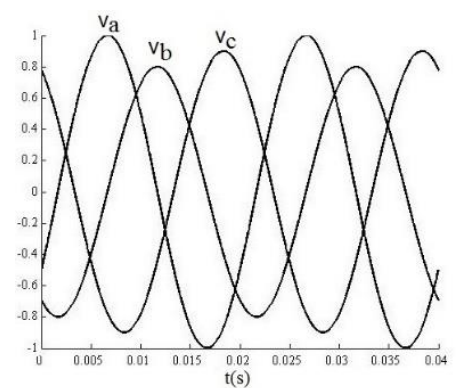

(b)

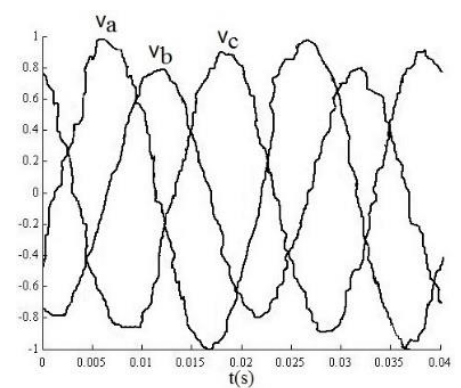

(c)

Figure 2.2: Three-phase voltage scenarios: (a) balanced, (b) unbalanced, (c) distorted and unbalanced

An unbalanced scenario takes place when the three-phase signals present different magnitudes or different shift angles among the phases, or both deviations together. This condition may happen due to asymmetric faults, for example. Additionally, the currents and the voltages may not be a perfect sine wave, typifying the presence of harmonic distortion. This condition may exist whereas the system is balanced or not, being typically caused by non-linear loads (arc furnaces, welding machines, etc) and power electronic equipment (switching action) [26, 27].

Adverse three-phase voltages and currents impose challenging conditions to properly define the power phenomena and evaluate the power being transferred. The main ideas related to unbalanced and distorted analyses are explained in sequence on this chapter, such as the mathematical transformations and the power theories developed to address these issues.

\section{2 - Vector Transformations}

The instantaneous power flowing through three-phase transmission systems depends on the relation among the instantaneous voltage and current on each conductor at the location being considered. 
However, analysis related to the voltage, current and power signals during imbalance and distortion is not as straightforward as during balanced conditions. For instance, when the system is not balanced, single line diagrams are no longer applicable and each phase must be individually represented.

For this reason, several mathematic transformations have been developed to assist on the visualization of complex waveforms in polyphase systems, employing decomposition techniques. Sections 2.2.1 until 2.2.4 present the main mathematical procedures, which can be generically represented for a three-phase voltage by:

$$
\left[\begin{array}{l}
V_{1} \\
V_{2} \\
V_{3}
\end{array}\right]=\left[\begin{array}{lll}
x_{11} & x_{12} & x_{13} \\
x_{21} & x_{22} & x_{23} \\
x_{31} & x_{32} & x_{33}
\end{array}\right]\left[\begin{array}{c}
V_{a} \\
V_{b} \\
V_{c}
\end{array}\right]
$$

and its inverse transformation, which leads back to the initial coordinate system as:

$$
\left[\begin{array}{l}
V_{a} \\
V_{b} \\
V_{c}
\end{array}\right]=\left[\begin{array}{lll}
y_{11} & y_{12} & y_{13} \\
y_{21} & y_{22} & y_{23} \\
y_{31} & y_{32} & y_{33}
\end{array}\right]\left[\begin{array}{c}
V_{1} \\
V_{2} \\
V_{3}
\end{array}\right]
$$

where the indexes $1,2,3$ are the new coordinates; $a, b, c$ the original components; and $x_{i j}$ and $y_{i j}$ the transformation elements, usually chosen to express particular physical relations within the power system performance or other peculiar characteristic that should be interesting to focus on.

\subsection{1 - The Symmetrical Components in the Frequency Domain}

The first ideas related to the symmetrical components in the frequency domain arose from decomposition techniques applied on electrical machines to study rotating magnetic fields, published by Tesla [28] and Ferraris [29] concomitantly in 1888. These 
methods were explored in a number of practical projects in the subsequent years after its statement, evolving to different approaches which decompose the rotating magnetic field in systems with two coordinates [30].

Nevertheless, the first formal publication of these techniques as a mathematic transformation was done by Stokvis in 1915, who proposed a method to represent unbalanced three-phase systems as an addition of other two balanced systems: the synchronous and the inverse systems, which later became known as positive and negative sequences, respectively [31].

The Manitoban Fortescue is regarded as the pioneer of electrical unbalanced network analysis. In 1918, he presented an elegant technique to represent $n$ unbalanced phasors by $n$ subsystems composed by $n$ balanced phasors [32]. His method was applied to three-phase systems, being referred as the renowned symmetrical components [33, 34], which includes a third component (zero sequence) in addition to the two components introduced by Stokvis.

The paper published by Fortescue was ranked as the highest impact paper in power engineering of all twentieth century [35]. As this is a University of Manitoba work, it is keen to note that Fortescue was a Manitoban whose father was a fur trading factor for the Hudson Bay Company in York Factory, Manitoba. Unfortunately, he did not graduate from the University of Manitoba, as there was no Electrical Engineering Department during his graduating time [36].

The symmetrical components enables any unbalanced three-phase phasors to be expressed by the addition of three sets of balanced symmetrical sequences [32-35]: the positive sequence (three phasors with $2 \pi / 3 \mathrm{rad}$ phase shift among each other, equal 
magnitude and same sequence as the original phasors); the negative sequence (three phasors as the positive sequence but in opposite sequence with the original phasors); and the zero sequence (three phasors of equal magnitude and zero angular displacement among them). A set of three-phase voltage phasors can be written as the sum of three voltage sequences as:

$$
\left[\begin{array}{c}
V_{a} \\
V_{b} \\
V_{c}
\end{array}\right]=\left[\begin{array}{c}
V_{a 0} \\
V_{b 0} \\
V_{c 0}
\end{array}\right]+\left[\begin{array}{c}
V_{a 1} \\
V_{b 1} \\
V_{c 1}
\end{array}\right]+\left[\begin{array}{c}
V_{a 2} \\
V_{b 2} \\
V_{c 2}
\end{array}\right]
$$

where the $a, b, c$ indexes refer to each phase of the original $a b c$ three-phase system and 0 , 1, 2 corresponds respectively to the zero, positive, and negative sequences.

The components of each sequence are mutually dependent, i.e. the components of phasors $b$ and $c$ can be written in terms of the components of phasor $a$ using the complex operator $\alpha$, which has unit length and $2 \pi / 3 \mathrm{rad}$ angle:

$$
\alpha=e^{j \frac{2 \pi}{3}}=-\frac{1}{2}+j \frac{\sqrt{3}}{2}
$$

Hence, Equation (2.5) may be written as:

$$
\left[\begin{array}{l}
V_{a} \\
V_{b} \\
V_{c}
\end{array}\right]=\left[\begin{array}{c}
V_{a 0} \\
V_{a 0} \\
V_{a 0}
\end{array}\right]+\left[\begin{array}{c}
V_{a 1} \\
\alpha^{2} V_{a 1} \\
\alpha V_{a 1}
\end{array}\right]+\left[\begin{array}{c}
V_{a 2} \\
\alpha V_{a 2} \\
\alpha^{2} V_{a 2}
\end{array}\right]=\left[\begin{array}{ccc}
1 & 1 & 1 \\
1 & \alpha^{2} & \alpha \\
1 & \alpha & \alpha^{2}
\end{array}\right]\left[\begin{array}{c}
V_{a 0} \\
V_{a 1} \\
V_{a 2}
\end{array}\right]
$$

The symmetrical components are obtained from the inverse transformation of Equation (2.7):

$$
\left[\begin{array}{c}
V_{a 0} \\
V_{a 1} \\
V_{a 2}
\end{array}\right]=\frac{1}{3}\left[\begin{array}{ccc}
1 & 1 & 1 \\
1 & \alpha & \alpha^{2} \\
1 & \alpha^{2} & \alpha
\end{array}\right]\left[\begin{array}{l}
V_{a} \\
V_{b} \\
V_{c}
\end{array}\right]
$$


Figure 2.3 illustrates a phasor diagram example with the positive, negative and zero sequence components, as well as the original phasors for each phase represented in a plane, i.e. in a two-dimensional space. It is possible to notice by superposition that the addition of the sequence components results in the original vectors.

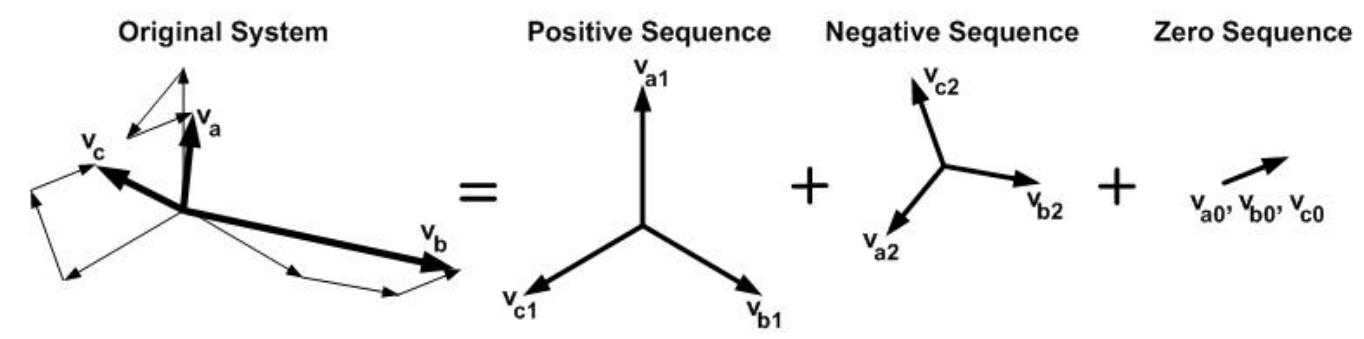

Figure 2.3: Symmetrical components of an unbalanced three-phase voltage

The elements of the transformation matrices on Equations (2.5) and (2.8) are all complex numbers (voltages and currents represented as phasors). Consequently, the symmetrical components are suitably applied to analyze quasi-stationary unbalanced conditions in the frequency domain.

\subsection{2 - The Symmetrical Components in the Time Domain}

The extended application of the symmetrical components in the time domain was introduced by Lyon in 1954 to accommodate dynamic unbalanced conditions [37]. His method is also commonly referred as instantaneous symmetrical components.

Both transformations, on the frequency and the time domains, are formally very similar, with the Lyon being evaluated at every instant for the voltage and the current. Fortescue's method can be considered a particular case of the instantaneous symmetrical components.

White and Woodson complemented the Lyon transformation proposing a power invariant form [38] represented by the following matrix: 


$$
L=\frac{1}{\sqrt{3}}\left[\begin{array}{ccc}
1 & 1 & 1 \\
1 & a^{2} & a \\
1 & a & a^{2}
\end{array}\right]
$$

and its inverse transformation defined as:

$$
L^{-1}=\frac{1}{\sqrt{3}}\left[\begin{array}{ccc}
1 & 1 & 1 \\
1 & a & a^{2} \\
1 & a^{2} & a
\end{array}\right]
$$

Essentially, the voltage and the current phasors are expressed by time dependent functions, with the complex operator $\alpha$ being replaced by the time shift operator $a$, exemplified in a backward shift equivalent to the operator of Equation (2.6) as:

$$
a(t) v(t)=v\left(t-\frac{4 \pi}{3}\right)
$$

where $a(t)$ refers to the time shift operator; and $v(t)$ a time dependent voltage signal. There are many digital forms to implement the time shift in practical systems, for example using adaptive filters, delays, or the H-transform [39-41].

The Lyon transformation first introduced the instantaneous phasors concept. It can be pictured as a middle ground between sinusoidal quasi-stationary modeling (e.g. phasors) and purely time domain representation (e.g. $\alpha \beta 0$ and the $d q 0$ representations).

\subsection{3 - The abO Transformation}

The $\alpha \beta 0$ components, although not so named, were first introduced in 1917 by Lewis [42] in a method to determine specific characteristics of the systems voltages and currents during line-to-ground faults. Such technique, yet unnamed, was employed in 
another work in 1931 to investigate the recovery voltage on circuit breakers after shortcircuits [43].

Clarke and Stanley first labelled this procedure as the $\alpha \beta 0$ transformation in 1938, employing to simplify the analysis of transient conditions on rotating machines $[44,45]$. At the same year, the $\alpha \beta 0$ method was mathematically formalized as modified symmetrical components by Clarke [46] and became known after her name, or simple the $\alpha \beta 0$ transformation. It is a space vector transformation of time dependent signals from the $a b c$ to the $\alpha \beta 0$ stationary coordinate systems. The transformation is mathematically given by the following matrix:

$$
C=\frac{2}{3}\left[\begin{array}{ccc}
1 & -\frac{1}{2} & -\frac{1}{2} \\
0 & \frac{\sqrt{3}}{2} & -\frac{\sqrt{3}}{2} \\
\frac{1}{2} & \frac{1}{2} & \frac{1}{2}
\end{array}\right]
$$

And its inverse transformation can be expressed by:

$$
C^{-1}=\left[\begin{array}{ccc}
1 & 0 & 1 \\
-\frac{1}{2} & \frac{\sqrt{3}}{2} & 1 \\
-\frac{1}{2} & -\frac{\sqrt{3}}{2} & 1
\end{array}\right]
$$

Considering the instantaneous components of the voltage and the current vectors represented in the $a b c$ reference frame as:

$$
\begin{aligned}
\vec{v}_{a b c} & =\left[\begin{array}{lll}
v_{a}(t) & v_{b}(t) & v_{c}(t)
\end{array}\right]^{T} \\
\vec{i}_{a b c} & =\left[\begin{array}{lll}
i_{a}(t) & i_{b}(t) & i_{c}(t)
\end{array}\right]^{T}
\end{aligned}
$$

and the same vectors represented on $\alpha \beta 0$ reference frame as: 


$$
\begin{aligned}
& \vec{v}_{\alpha \beta 0}=\left[\begin{array}{lll}
v_{\alpha}(t) & v_{\beta}(t) & v_{0}(t)
\end{array}\right]^{T} \\
& \vec{i}_{\alpha \beta 0}=\left[\begin{array}{lll}
i_{\alpha}(t) & i_{\beta}(t) & i_{0}(t)
\end{array}\right]^{T}
\end{aligned}
$$

The $\alpha \beta 0$ components of the three-phase voltage and current can be evaluated by:

$$
\begin{gathered}
\vec{v}_{\alpha \beta 0}=C \vec{v}_{a b c} \\
\vec{i}_{\alpha \beta 0}=C \vec{i}_{a b c}
\end{gathered}
$$

and the inverse calculation from $\alpha \beta 0$ to $a b c$ can be expressed as:

$$
\begin{aligned}
\vec{v}_{a b c} & =C^{-1} \vec{v}_{\alpha \beta 0} \\
\vec{i}_{a b c} & =C^{-1} \vec{i}_{\alpha \beta 0}
\end{aligned}
$$

The transformations (2.18) and (2.19) separate the non-homopolar modes (positive and negative sequences) from the homopolar mode (zero sequence). The homopolar current is usually not considered on steady-state analyses. Thus, it is common to represent the transformation matrices without the line or the column associated with the zero sequence (reduced Clarke transformation) [47].

The original transformations proposed by Equations (2.18) and (2.19) are not power invariant and it gives the same zero sequence values as the original method of the symmetrical components. Concordia adjusted the $\alpha \beta 0$ transformation to the unitary power invariant form [48], being expressed as:

$$
C_{\alpha \beta 0}=\sqrt{\frac{2}{3}}\left[\begin{array}{ccc}
1 & -\frac{1}{2} & -\frac{1}{2} \\
0 & \frac{\sqrt{3}}{2} & -\frac{\sqrt{3}}{2} \\
\frac{1}{\sqrt{2}} & \frac{1}{\sqrt{2}} & \frac{1}{\sqrt{2}}
\end{array}\right]
$$

with the inverse transformation given by: 


$$
C_{\alpha \beta 0}^{-1}=\sqrt{\frac{2}{3}}\left[\begin{array}{ccc}
1 & 0 & \frac{1}{\sqrt{2}} \\
-\frac{1}{2} & \frac{\sqrt{3}}{2} & \frac{1}{\sqrt{2}} \\
-\frac{1}{2} & -\frac{\sqrt{3}}{2} & \frac{1}{\sqrt{2}}
\end{array}\right]
$$

From the matrix elements of equations (2.22) and (2.23), it is possible to observe trigonometric values associated with the angle $2 \pi / 3 \mathrm{rad}$, allowing the reduced form of the matrix transformation (2.22) to be rewritten as:

$$
\left[\begin{array}{l}
v_{\alpha}(t) \\
v_{\beta}(t)
\end{array}\right]=\sqrt{\frac{2}{3}}\left[\begin{array}{ccc}
\cos 0 & \cos \left(\frac{2 \pi}{3}\right) & \cos \left(-\frac{2 \pi}{3}\right) \\
\sin 0 & \sin \left(\frac{2 \pi}{3}\right) & \sin \left(-\frac{2 \pi}{3}\right)
\end{array}\right]\left[\begin{array}{l}
v_{a}(t) \\
v_{b}(t) \\
v_{c}(t)
\end{array}\right]
$$

Equation (2.24) can be graphically represented in a two-dimensional coordinate system referred as the Clarke plane, or the $\alpha \beta$ plane (Figure 2.4), with the voltage and the current vectors represented as:

$$
\begin{gathered}
\vec{v}_{\alpha \beta}=v_{\alpha}(t) \hat{\alpha}+v_{\beta}(t) \hat{\beta} \\
\vec{i}_{\alpha \beta}=i_{\alpha}(t) \hat{\alpha}+i_{\beta}(t) \hat{\beta}
\end{gathered}
$$

where $\hat{\alpha}$ and $\hat{\beta}$ are the unit vectors in the $\alpha \beta$ cartesian coordinate plane. These voltage and current vectors are time dependent, and they should not be misinterpreted as phasors.

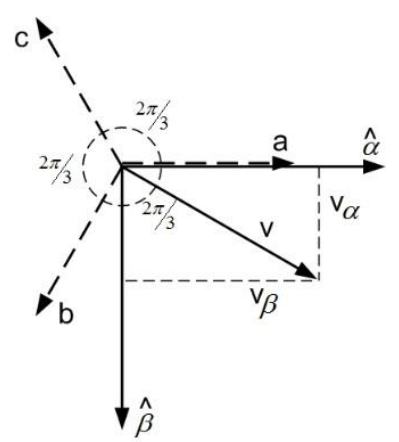

Figure 2.4: The abc phasors and the $\alpha \beta$ unit vectors represented in a two-dimension cartesian plane 


\subsection{4 - The $d q O$ Transformation}

The $d q 0$ transformation was introduced earlier than the $\alpha \beta 0$ transformation by Park in his influential paper on synchronous machine published in 1929 [49], which ranked second at the evaluation of the high impact papers in power engineering of the twentieth century [36]. It establishes the relation between the stationary $a b c$ coordinate system and the rotating $d q 0$ coordinate system.

However, conceptually, it can be understood as an expanded version of the idea proposed by Clarke, with a time dependent angle shift transformation that converts the stationary to the synchronous rotating frame. The synchronous rotation takes place in the $d q$ plane around the rotation axis 0 . It can be aligned to the voltage, the current, or any other vector reference as an initial phase shift.

As in the original Clarke transformation, the zero sequence calculated by the Park transformation is the same as the zero sequence component obtained using the symmetrical components proposed by Fortescue. It is important to notice that there is no tracking reference for the zero sequence component, as there are for the positive and negative components.

The Park transformation is expressed by:

$$
P=\sqrt{\frac{2}{3}}\left[\begin{array}{ccc}
\cos \delta & \cos \left(\delta-\frac{2 \pi}{3}\right) & \cos \left(\delta+\frac{2 \pi}{3}\right) \\
-\sin \delta & -\sin \left(\delta-\frac{2 \pi}{3}\right) & -\sin \left(\delta+\frac{2 \pi}{3}\right) \\
\frac{1}{\sqrt{2}} & \frac{1}{\sqrt{2}} & \frac{1}{\sqrt{2}}
\end{array}\right]
$$

and the inverse Park transformation can be calculated by the expression: 


$$
P^{-1}=\sqrt{\frac{2}{3}}\left[\begin{array}{ccc}
\cos \delta & -\sin \delta & \frac{1}{\sqrt{2}} \\
\cos \left(\delta-\frac{2 \pi}{3}\right) & -\sin \left(\delta-\frac{2 \pi}{3}\right) & \frac{1}{\sqrt{2}} \\
\cos \left(\delta+\frac{2 \pi}{3}\right) & -\sin \left(\delta+\frac{2 \pi}{3}\right) & \frac{1}{\sqrt{2}}
\end{array}\right]
$$

where $\delta=\omega t+\delta_{i}$ is the angle between the rotating and the fixed coordinate system and $\delta_{i}$ corresponds to the initial phase shift.

For three-phase balanced systems, the compact Park transformation (also named $d q$ transformation, omitting the zero sequence elements) presents advantageous features related to the reduction of three-phase AC variables in two DC variables [50], aiding for example on the design of filters and controllers for voltage sourced converters (concepts further explored in Chapter 4).

After considering the previous main mathematic tools developed to analyze adverse AC voltage and currents conditions, this work moves forward towards the evaluation of the instantaneous power in three-phase transmission lines.

\section{3 - Instantaneous Power Theories}

In power systems, the electric power can be defined as the rate at which electric energy is transferred per time unit through a given cross section of a transmission line. The instantaneous power concept emerges when the time period observed during the energy flow becomes infinitesimal, representing the power at each instant. And a power theory is the set of power definitions, the explanation of its properties, the relationship among these concepts and their physical interpretations, combining mathematics, physics and technology models. 


\subsection{1 - Classical Power Definitions}

Conventional power definitions are well established for single-phase systems under sinusoidal conditions [51]. Considering a single-phase circuit with sinusoidal voltage and current signals:

$$
\begin{gathered}
v(t)=V \sin \left(\omega t+\phi_{v}\right) \\
i(t)=I \sin \left(\omega t+\phi_{i}\right)
\end{gathered}
$$

the instantaneous power is mathematically defined as:

$$
p(t)=v(t) i(t)
$$

Substituting Equations (2.29) and (2.30) on (2.31), after simplifications, the power in a single-phase system can be expressed as:

$$
p(t)=\underbrace{\overbrace{V_{r m s} I_{r m s} \cos \left(\phi_{v}-\phi_{i}\right)}^{P}[1-\cos (2 \omega t)]}_{I}-\underbrace{\overbrace{V_{r m s} I_{r m s} \sin \left(\phi_{v}-\phi_{i}\right)}^{Q} \sin (2 \omega t)}_{I I}
$$

where $V_{r m s}$ and $I_{r m s}$ represents the respective $r m s$ values (root mean square) of the voltage and the current.

The expression (2.32) shows two oscillating terms at twice the system frequency. The active power $P$ (also called real power) is the average value of the instantaneous active power (term $I$ ). Moreover, the reactive power $Q$ is the peak value of the instantaneous reactive power (term II). Figure 2.5 illustrates the waveforms related to these power definitions. 


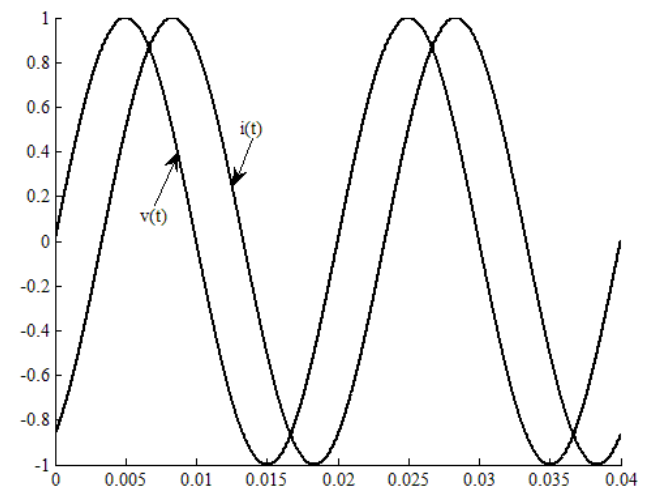

(a)

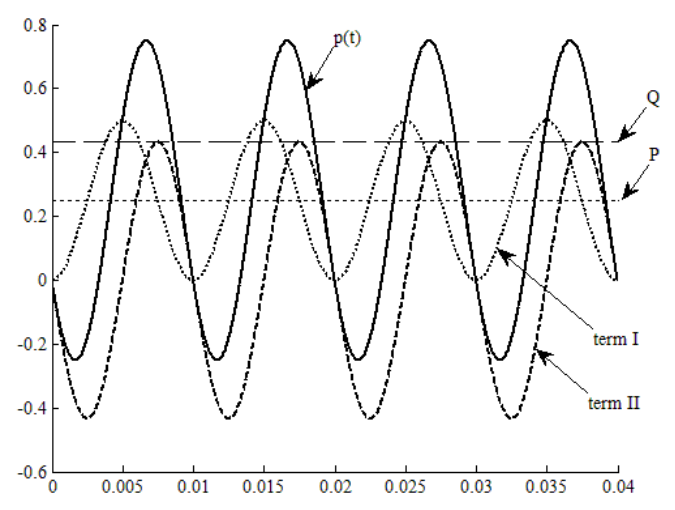

(b)

Figure 2.5: Single-phase power: (a) voltage and current, $\phi_{v}-\phi_{i}=\pi / 3$, (b) single-phase power components

The instantaneous active power oscillates, being always present whenever energy is transferred. The active power is always positive (considering $\pi / 2 \leq \phi_{v}-\phi_{i} \leq \pi / 2$ ), representing an unidirectional power flow. The instantaneous reactive power appears when the current and the voltage are not in phase. It presents zero average value and it is usually interpreted as the oscillating power which is constantly produced and consumed.

The power theory developed for single-phase circuits can be extended to threephase systems. The three-phase power is calculated by adding the single-phase power contributions as:

$$
p_{3 \phi}(t)=v_{a}(t) i_{a}(t)+v_{b}(t) i_{b}(t)+v_{c}(t) i_{c}(t)
$$

Substituting Equations 2.1 and 2.2 on 2.33, after simplifications:

$$
\begin{aligned}
p_{3 \phi}(t)=V_{r m s} I_{r m s}\left[\cos \left(\phi_{v}-\phi_{i}\right)-\cos \left(2 \omega t+\phi_{v}+\phi_{i}\right)+\right. \\
+\cos \left(\phi_{v}-\phi_{i}\right)-\cos \left(2 \omega t+\phi_{v}+\phi_{i}+\frac{2 \pi}{3}\right)+ \\
\left.+\cos \left(\phi_{v}-\phi_{i}\right)-\cos \left(2 \omega t+\phi_{v}+\phi_{i}-\frac{2 \pi}{3}\right)\right]
\end{aligned}
$$


Additionally, when the system is balanced, Equation (2.34) can be simplified, providing the expression:

$$
p_{3 \phi}(t)=3 V_{r m s} I_{r m s} \cos \left(\phi_{v}-\phi_{i}\right)
$$

Equation (2.35) shows that the energy transfer is constant when the system is balanced. This condition helps, for instance, to mitigate undesirable mechanical vibrations on machines connected to the electrical network (generators, motors). According to the Kirchhoff's circuit law, the instantaneous three-phase currents cancel each other on the return path, resulting in zero ground or neutral current, as mentioned earlier in this chapter.

In a similar way, the three-phase reactive power for balanced conditions can be derived by extending the concepts of the single-phase power:

$$
q_{3 \phi}(t)=3 V_{r m s} I_{r m s} \sin \left(\phi_{v}-\phi_{i}\right)
$$

However, the reactive power definition does not share the same physical meaning of the single-phase circuits. Unfortunately, there is no accurate physical interpretation for three-phase systems regarding this portion of energy which does not produce power. In addition, this issue becomes more complex when the system is not balanced, the classic reactive power interpretations cannot be easily applied $[52,53]$.

Therefore, classical power theory concepts cannot be simply extended to general AC situations. The precise evaluation of the instantaneous power to encompass unbalanced and distorted circumstances demands further advanced developments.

\subsection{2 - Power Concepts under Adverse Conditions}

The first power theories dealing with adverse AC scenarios emerged around the end of the nineteenth century. The discussions were sparked by observing that the apparent 
power could be higher than the active power for non-sinusoidal conditions. The investigations were conducted following two distinct trends: analyses in the frequency and in the time domain.

The power theory proposed by Budeanu in 1927 [54] was the most widespread concept in the frequency domain during the early studies. It launched the concept of distorted power in electric networks, defined by the expression:

$$
S=\sqrt{P^{2}+Q^{2}+D^{2}}
$$

where $S, P, Q$ and $D$ corresponds respectively to the apparent, active, reactive and distorted powers.

The Budeanu's theory introduces the distorted power to create a balance in the power equation. However, this portion of power does not have straight physical attributes related to any specific phenomenon on the electric circuit. It is interpreted only as an increase of the apparent power due to the waveform distortion.

Budeanu's definitions were demonstrated to be partially erroneous [55], although it is still supported by the IEEE Standards [56]. The reactive and distorted powers are not associated with energy oscillation nor current distortion. Nevertheless, it must be recognized as the first theory to point out the need to create new power concepts to represent different properties of the system under non-sinusoidal conditions.

Later on, another approach emerged in 1932, defining power properties in the time domain. Fryze proposed pioneering ideas for the current decomposition into active and reactive components [57], being mathematically represented by:

$$
i(t)=i_{a}(t)+i_{r f}(t)
$$


with the components mutually orthogonal to each other and, therefore, able to be expressed as:

$$
i^{2}(t)=i_{a}^{2}(t)+i_{r f}^{2}(t)
$$

Fryze's theory states that the active power is related to the portion of the current that is in phase with the fundamental voltage, $i_{a}(t)$, and the reactive power comprises all the fractions of the current which does not contribute to the active power, $i_{r f}(t)$. The theory proposed by Fryze also presents interpretation flaws, for instance, in regards to the reactive current and reactive power which cannot be associated with any phenomenon in the load.

Buchholz and Depenbrock made important contributions related to the decomposition of the current, enhancing the ideas exposed by Fryze in the time domain. The FBD method (named after Frize, Buchholz and Depenbrock) presents instantaneous calculation of the active current components for generic multiphase system [58].

Essentially, Budeanu and Fryze proposed their original theories exclusive for single-phase circuits, respectively, in the frequency and the time domain. Afterwards, their innovative concepts were expanded to three-phase circuits. But they were not capable to explain completely the power phenomena for general adverse AC conditions, neither able to provide accurate means to design power compensation methods for three-phase systems.

These first theories set the base for an expansion into advanced power concepts, contributing to establish other remarkable approaches. Throughout the following decades of the twentieth century, enhanced power concepts had been proposed in an attempt to clarify the power phenomena for general AC conditions.

Among the modern concepts, there are two foremost theories, both developed in the time domain: the CPC (Current Physical Components) enounced by Czarneck [59, 60], 
originally proposed in 1983; and the $p-q$ theory developed by Akagi [61, 62], with its first related publication in 1982 .

They are currently the most sophisticated power theories proposed for power systems which have been accepted by top scientific communities to explain phenomena related to non-sinusoidal voltages and currents. Additionally, both theories have been constantly improved with numerous complementary papers published by prestigious institutions worldwide.

The main feature of the Current Physical Components theory is the association of current components with physical phenomena. It shares the same concept for the active current $i_{a}(t)$ as proposed by Fryze. The remaining non-active components are decomposed in scattered current $i_{s}(t)$ and reactive current $i_{r}(t)$ :

$$
i(t)=i_{a}(t)+i_{s}(t)+i_{r}(t)
$$

which are mutually orthogonal and, therefore, they can be represented by:

$$
i^{2}(t)=i_{a}^{2}(t)+i_{s}^{2}(t)+i_{r}^{2}(t)
$$

They are associated with three distinct physical phenomena: permanent energy conversion (active current), change of the load impedance with the harmonic order (scattered current), and phase shift between voltage and current harmonics (reactive current). And when multiplied by the voltage leads to the following power equation:

$$
S=\sqrt{P^{2}+D_{s}^{2}+Q^{2}}
$$

where $S, P, D_{s}$ and $Q$ corresponds respectively to the apparent, active, scattered and reactive powers. 
On the other side, the $p-q$ theory elegantly emerged and rapidly became very well adopted by large group of professionals specialized in power electronics devices. It is a powerful theory which have been successfully applied to design power compensation schemes for voltage sourced converters operating under adverse conditions, topic strongly related to the objective of the present work.

The $p-q$ theory introduces the instantaneous imaginary power concept, explaining its association with the exchange of energy among each phase of the three-phase three-wire transmission section, due to the non-active currents flowing through the conductors. Figure 2.6 illustrates the real and imaginary power concepts proposed by Akagi.

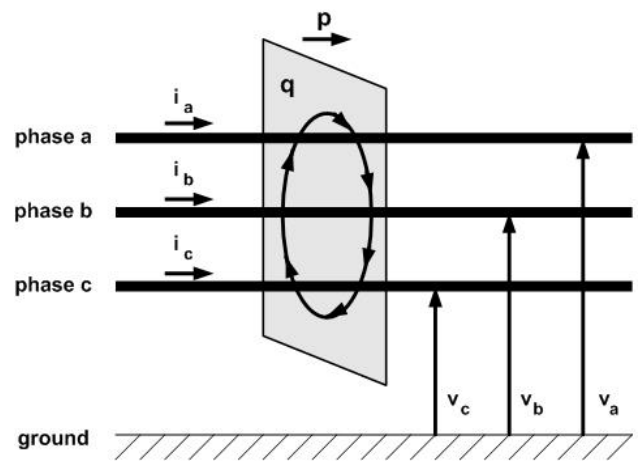

Figure 2.6: Physical interpretation of the p-q theory

Over the last decades, the original $p$ - $q$ theory has also been complemented by several publications; the most well-known worth mentioning is the modified $p$ - $q$ theory, employed on the design of controllers for power electronics devices [63, 64].

However, considering adverse AC scenarios with three independent voltages and three independent currents, the $p-q$ theory and the modified $p-q$ theory do not allow to decompose the calculation of the total active and reactive power in the form:

$$
\begin{aligned}
& p(t)=\bar{p}(t)+\tilde{p}(t) \\
& q(t)=\bar{q}(t)+\tilde{q}(t)
\end{aligned}
$$


where $\bar{p}(t)$ and $\bar{q}(t)$ correspond to the constant terms of the active and reactive powers, respectively; and $\tilde{p}(t)$ and $\tilde{q}(t)$ refer to the oscillating terms of the active and reactive powers, respectively; which all together express the total instantaneous power.

The present work proposes an innovative method able to independently evaluate the terms associated to Equations (2.43) and (2.44) during adverse AC scenarios, including zero sequence voltage and current content. No work has ever been published presenting such feature, which became possible by the development of new mathematical transformations and power equations, summarized as the mno instantaneous power theory (Chapter 3).

The mno power theory makes possible the development and design of the mno instantaneous power components control, an enhanced power control technique for voltage sourced converters proposed (Chapter 4). Before introducing the mno theory, the $p-q$ theory equations are presented in the next section.

\section{4 - The $p-q$ Instantaneous Power Theory}

The $p-q$ theory is based on expressing voltages and currents as time dependent vectors by using the $\alpha \beta 0$ transformation (Section 2.2.3). This theory was first proposed for three-phase three-wire systems and it was later expanded to three-phase four-wire systems. 


\subsection{1 - Three-Phase Three-Wire Systems}

The definition of the instantaneous active and reactive power for a three-phase three-wire system is expressed respectively by the following equations:

$$
\begin{aligned}
& p(t)=v_{\alpha}(t) i_{\alpha}(t)+v_{\beta}(t) i_{\beta}(t) \\
& q(t)=v_{\beta}(t) i_{\alpha}(t)-v_{\alpha}(t) i_{\beta}(t)
\end{aligned}
$$

which can be represented in the matrix form as:

$$
\left[\begin{array}{l}
p(t) \\
q(t)
\end{array}\right]=\underbrace{\left[\begin{array}{cc}
v_{\alpha}(t) & v_{\beta}(t) \\
v_{\beta}(t)-v_{\alpha}(t)
\end{array}\right]}_{V_{p q}} \cdot\left[\begin{array}{l}
i_{\alpha}(t) \\
i_{\beta}(t)
\end{array}\right]
$$

The current terms can be evaluated as:

$$
\left[\begin{array}{c}
i_{\alpha}(t) \\
i_{\beta}(t)
\end{array}\right]=V_{p q}^{-1} \cdot\left[\begin{array}{c}
p(t) \\
q(t)
\end{array}\right]=\frac{1}{v_{\alpha}^{2}(t)+v_{\beta}^{2}(t)}\left[\begin{array}{cc}
v_{\alpha}(t) & v_{\beta}(t) \\
v_{\beta}(t) & -v_{\alpha}(t)
\end{array}\right] \cdot\left[\begin{array}{l}
p(t) \\
q(t)
\end{array}\right]
$$

which can be rewritten as compounded by the following components:

$$
\left[\begin{array}{c}
i_{\alpha}(t) \\
i_{\beta}(t)
\end{array}\right]=\left[\begin{array}{c}
i_{\alpha p}(t) \\
i_{\beta p}(t)
\end{array}\right]+\left[\begin{array}{l}
i_{\alpha q}(t) \\
i_{\beta q}(t)
\end{array}\right]
$$

where each term of Equation (2.49) is calculated as:

$$
\begin{array}{ll}
i_{\alpha p}(t)=\frac{v_{\alpha}(t)}{v_{\alpha}^{2}(t)+v_{\beta}^{2}(t)} p(t) & i_{\alpha q}(t)=\frac{v_{\beta}(t)}{v_{\alpha}^{2}(t)+v_{\beta}^{2}(t)} q(t) \\
i_{\beta p}(t)=\frac{v_{\beta}(t)}{v_{\alpha}^{2}(t)+v_{\beta}^{2}(t)} p(t) & i_{\beta q}(t)=\frac{-v_{\alpha}(t)}{v_{\alpha}^{2}(t)+v_{\beta}^{2}(t)} q(t)
\end{array}
$$

Thus, Equation (2.47) can be written with separated power contributions as:

$$
\left[\begin{array}{c}
p(t) \\
q(t)
\end{array}\right]=\left[\begin{array}{cc}
v_{\alpha}(t) & v_{\beta}(t) \\
v_{\beta}(t) & -v_{\alpha}(t)
\end{array}\right] \cdot\left[\begin{array}{c}
i_{\alpha p}(t)+i_{\alpha q}(t) \\
i_{\beta p}(t)+i_{\beta q}(t)
\end{array}\right]
$$




$$
\begin{gathered}
p(t)=v_{\alpha}(t) i_{\alpha p}(t)+v_{\alpha}(t) i_{\alpha q}(t)+v_{\beta}(t) i_{\beta p}(t)+v_{\beta}(t) i_{\beta q}(t) \\
p(t)=p_{\alpha p}(t)+p_{\alpha q}(t)+p_{\beta p}(t)+p_{\beta q}(t) \\
p(t)=\frac{v_{\alpha}^{2}(t)}{v_{\alpha}^{2}(t)+v_{\beta}^{2}(t)} p(t)+\frac{v_{\alpha}(t) v_{\beta}(t)}{v_{\alpha}^{2}(t)+v_{\beta}^{2}(t)} q(t)+\frac{v_{\beta}^{2}(t)}{v_{\alpha}^{2}(t)+v_{\beta}^{2}(t)} p(t)+\frac{-v_{\alpha}(t) v_{\beta}(t)}{v_{\alpha}^{2}(t)+v_{\beta}^{2}(t)} q(t)
\end{gathered}
$$

and the instantaneous reactive power can also be represented by the separated components:

$$
\begin{gathered}
q(t)=v_{\beta}(t) i_{\alpha p}(t)+v_{\beta}(t) i_{\alpha q}(t)-v_{\alpha}(t) i_{\beta p}(t)-v_{\alpha}(t) i_{\beta q}(t) \\
q(t)=\frac{v_{\alpha}(t) v_{\beta}(t)}{v_{\alpha}^{2}(t)+v_{\beta}^{2}(t)} p(t)+\frac{v_{\beta}^{2}(t)}{v_{\alpha}^{2}(t)+v_{\beta}^{2}(t)} q(t)-\frac{v_{\alpha}(t) v_{\beta}(t)}{v_{\alpha}^{2}(t)+v_{\beta}^{2}(t)} p(t)+\frac{v_{\alpha}^{2}(t)}{v_{\alpha}^{2}(t)+v_{\beta}^{2}(t)} q(t)
\end{gathered}
$$

The instantaneous current is divided into active and reactive components in both axis $\alpha$ and $\beta$ of the Clarke plane. The sum of the $\alpha$ axis and the $\beta$ axis active power components corresponds to the instantaneous active power. The sum of the $\alpha$ axis and the $\beta$ axis reactive power components is always zero, with contribution to neither instantaneous nor the average energy flow between source and load; their magnitude is associated with the instantaneous reactive power, which represents the quantity of energy that is being exchanged among the phases of the system.

\subsection{2 - Three-Phase Four-Wire Systems}

The three line currents are independent of each other in a three-phase four-wire system. Therefore, the zero sequence current components are allowed to flow through the four-wire transmission topology. In this case, the $p-q$ theory is mathematically defined as:

$$
\left[\begin{array}{c}
p(t) \\
q(t) \\
p_{0}(t)
\end{array}\right]=\left[\begin{array}{ccc}
v_{\alpha}(t) & v_{\beta}(t) & 0 \\
v_{\beta}(t) & -v_{\alpha}(t) & 0 \\
0 & 0 & v_{0}(t)
\end{array}\right] \cdot\left[\begin{array}{c}
i_{\alpha}(t) \\
i_{\beta}(t) \\
i_{0}(t)
\end{array}\right]
$$


The active and reactive powers have the same meaning compared to the $p$ - $q$ theory in three-phase three-wire systems and the zero sequence power is considered completely independent from the previous power terms. For example, Equation (2.45) is rewritten with the addition of the zero sequence power contribution as:

$$
p(t)=v_{\alpha}(t) i_{\alpha}(t)+v_{\beta}(t) i_{\beta}(t)+v_{0}(t) i_{0}(t)
$$

The zero sequence power has the same characteristics as the instantaneous power in a single-phase circuit (Section 2.2.1), with an average value $\bar{p}_{o}$ and an oscillating value $\tilde{p}_{o}$ at twice the frequency. The $p-q$ theory states that it is impossible to produce constant zero sequence power alone, i.e., the average and oscillating terms of the active zero sequence power are always linked to one another [65].

\subsection{3 - The Modified $p-q$ Theory}

The active power can be represented as the scalar product of the instantaneous vectors of the voltage and the current, and the reactive power can be expressed as the cross product of these vectors. Thus, the total instantaneous power can be mathematically written as:

$$
\begin{aligned}
& p(t)=\vec{v}_{\alpha \beta 0}(t) \cdot \vec{i}_{\alpha \beta 0}(t)=v_{\alpha}(t) i_{\alpha}(t)+v_{\beta}(t) i_{\beta}(t)+v_{0}(t) i_{0}(t) \\
& \vec{q}(t)=\vec{v}_{\alpha \beta 0}(t) \times \vec{i}_{\alpha \beta 0}(t)=q_{\alpha}(t) \hat{\alpha}+q_{\beta}(t) \hat{\beta}+q_{0}(t) \hat{0}
\end{aligned}
$$

where the reactive power components on each direction are:

$$
\begin{aligned}
& \vec{q}_{\alpha}(t)=\left|\begin{array}{ll}
v_{\beta}(t) & v_{0}(t) \\
i_{\beta}(t) & i_{0}(t)
\end{array}\right| \hat{\alpha}=\left[v_{\beta}(t) i_{0}(t)-v_{0}(t) i_{\beta}(t)\right] \hat{\alpha} \\
& \vec{q}_{\beta}(t)=\left|\begin{array}{ll}
v_{0}(t) & v_{\alpha}(t) \\
i_{0}(t) & i_{\alpha}(t)
\end{array}\right| \hat{\beta}=\left[v_{0}(t) i_{\alpha}(t)-v_{\alpha}(t) i_{0}(t)\right] \hat{\beta}
\end{aligned}
$$




$$
\vec{q}_{0}(t)=\left|\begin{array}{cc}
v_{\alpha}(t) & v_{\beta}(t) \\
i_{\alpha}(t) & i_{\beta}(t)
\end{array}\right| \hat{0}=\left[v_{\alpha}(t) i_{\beta}(t)-v_{\beta}(t) i_{\alpha}(t)\right] \hat{0}
$$

The instantaneous active power and instantaneous reactive power of Equations (2.60) and (2.61) can also be defined in a matrix form:

$$
\left[\begin{array}{c}
p(t) \\
q_{\alpha}(t) \\
q_{\beta}(t) \\
q_{0}(t)
\end{array}\right]=\underbrace{\left[\begin{array}{ccc}
v_{\alpha}(t) & v_{\beta}(t) & v_{0}(t) \\
0 & -v_{0}(t) & v_{\beta}(t) \\
v_{0} & 0 & -v_{\alpha}(t) \\
-v_{\beta}(t) & v_{\alpha}(t) & 0
\end{array}\right]}_{v_{p q}}\left[\begin{array}{c}
i_{\alpha}(t) \\
i_{\beta}(t) \\
i_{0}(t)
\end{array}\right]
$$

and the three-phase total instantaneous reactive power in terms of $\alpha \beta 0$ components is evaluated as the magnitude of the reactive vector:

$$
q(t)=|\vec{q}(t)|=\sqrt{q_{\alpha}(t)^{2}+q_{\beta}(t)^{2}+q_{0}(t)^{2}}
$$

Moreover, by applying the same procedure used for three-phase three-wire systems, the current on the modified $p-q$ theory can be evaluated from (2.65) by using the inverse mathematical operation:

$$
\left[\begin{array}{c}
i_{\alpha}(t) \\
i_{\beta}(t) \\
i_{0}(t)
\end{array}\right]=V_{p q}^{-1}\left[\begin{array}{c}
p(t) \\
q_{\alpha}(t) \\
q_{\beta}(t) \\
q_{0}(t)
\end{array}\right]=\frac{1}{v_{\alpha}^{2}(t)+v_{\beta}^{2}(t)+v_{0}^{2}(t)}\left[\begin{array}{cccc}
v_{\alpha}(t) & 0 & v_{0} & -v_{\beta}(t) \\
v_{\beta}(t) & -v_{0}(t) & 0 & v_{\alpha}(t) \\
v_{0}(t) & v_{\beta}(t) & -v_{\alpha}(t) & 0
\end{array}\right]\left[\begin{array}{c}
p(t) \\
q_{\alpha}(t) \\
q_{\beta}(t) \\
q_{0}(t)
\end{array}\right]
$$

Then, for each one of the currents, the active and reactive components can also be derived. The instantaneous active current on the $\alpha$ axis:

$$
i_{\alpha p}(t)=\frac{v_{\alpha}(t)}{v_{\alpha}^{2}(t)+v_{\beta}^{2}(t)+v_{0}^{2}(t)} p(t)
$$

the instantaneous active current on the $\beta$ axis: 


$$
i_{\beta p}(t)=\frac{v_{\beta}(t)}{v_{\alpha}^{2}(t)+v_{\beta}^{2}(t)+v_{0}^{2}(t)} p(t)
$$

the instantaneous zero-sequence active current:

$$
i_{0 p}(t)=\frac{v_{0}(t)}{v_{\alpha}^{2}(t)+v_{\beta}^{2}(t)+v_{0}^{2}(t)} p(t)
$$

the instantaneous reactive current on the $\alpha$ axis:

$$
i_{\alpha q}(t)=\frac{v_{0}(t)}{v_{\alpha}^{2}(t)+v_{\beta}^{2}(t)+v_{0}^{2}(t)} q_{\beta}(t)-\frac{v_{\beta}(t)}{v_{\alpha}^{2}(t)+v_{\beta}^{2}(t)+v_{0}^{2}(t)} q_{0}(t)
$$

the instantaneous reactive current on the $\beta$ axis:

$$
i_{\beta q}(t)=\frac{v_{\alpha}(t)}{v_{\alpha}^{2}(t)+v_{\beta}^{2}(t)+v_{0}^{2}(t)} q_{0}(t)-\frac{v_{0}(t)}{v_{\alpha}^{2}(t)+v_{\beta}^{2}(t)+v_{0}^{2}(t)} q_{\alpha}(t)
$$

and the instantaneous reactive current on zero-sequence axis as:

$$
i_{0 q}(t)=\frac{v_{\beta}(t)}{v_{\alpha}^{2}(t)+v_{\beta}^{2}(t)+v_{0}^{2}(t)} q_{\alpha}(t)-\frac{v_{\alpha}(t)}{v_{\alpha}^{2}(t)+v_{\beta}^{2}(t)+v_{0}^{2}(t)} q_{\beta}(t)
$$

Equations (2.68) to (2.73) also do not allow decomposing the total instantaneous power into constant and oscillating terms in the presence of positive, negative and zero sequence currents and, therefore, the design of power converter control methods becomes compromised in order to produce only constant power during adverse AC conditions with zero sequence voltage and current content.

This is because the $p-q$ theory and its modified complementary versions are attached to the Clarke's transformation. To overcome this issue, the present work proposes the novel mno transformation (Chapter 3). 


\section{Chapter 3}

\section{The mno Instantaneous Power Theory}

This chapter introduces a new adaptive transformation method, labelled mno transformation, which allows the decomposition of the total instantaneous power flowing on general three-phase transmission topologies into constant and oscillating terms, referred to as the mno instantaneous power components. It is applied to three-wire and four-wire schemes, especially dealing with zero sequence power unlike previous methods. It extends and improves the application of the $p$ - $q$ theory concepts, which are based on the stationary $\alpha \beta 0$ transformation.

\section{1 - The Three-Dimensional Time Domain}

\section{Reference Frame}

The classical stationary phasors diagrams are typically employed on time invariant analyses of sinusoidal signals. They are not suitable to analyze transient voltages and currents, notwithstanding steady-state extensions [66]. The dynamic phasors appeared as one solution to overcome this issue, embracing time variant events by using time dependent 
Fourier coefficients [67]. However, the instantaneous approach is limited due to the time window needed for its generalized averaging procedure, with restricted bandwidth.

On the other side, time dependent vectors better suit instantaneous analyses for voltages and currents. They can be graphically represented in reference frames by different coordinate systems, illustrating the instantaneous state of the three-phase voltage and current. For example, the transformations proposed by Clarke and Park (Chapter 2) can be graphically illustrated on two-dimensional cartesian coordinates, respectively the $\alpha \beta$ and $d q$ planes, representing the three-phase instantaneous voltage and current vectors. For the majority of the studies, these coordinate systems are employed without the zero sequence representation (reduced transformation form, Section 2.2.3 and Section 2.2.4), which is convenient for instantaneous power flow analyses in three-wire systems.

However, even when representing zero sequence content, Park's transformation, for example, does not include an oscillating nor a rotating reference to track the zero sequence component, as it tracks the positive and negative sequence components in the $d q$ plane, i.e. as shown in the Section 2.2.4 the angular reference for the zero sequence is stationary in the synchronous Park transformation.

The coordinate systems should be able to represent magnitudes, phase angles and frequencies of the voltage and the current at each phase of the three-phase system during any dynamic condition, i.e. considering positive, negative and zero sequence content transients.

The three independent voltages and three independent currents of a general threephase configuration can be instantaneously represented in an $a b c$ three-dimensional cartesian coordinate system by the following time dependent vectors: 


$$
\begin{gathered}
\vec{v}_{a b c}(t)=v_{a}(t) \hat{a}+v_{b}(t) \hat{b}+v_{c}(t) \hat{c} \\
\vec{i}_{a b c}(t)=i_{a}(t) \hat{a}+i_{b}(t) \hat{b}+i_{c}(t) \hat{c}
\end{gathered}
$$

where $\hat{a}, \hat{b}$ and $\hat{c}$ are the set of directional unit vectors of the cartesian coordinate basis representing respectively phases $a, b$ and $c$. These vectors can be equally expressed by matrices where each element corresponds to one scalar component of the three-dimensional $a b c$ reference as:

$$
\begin{aligned}
& \vec{v}_{a b c}(t)=\left[\begin{array}{lll}
v_{a}(t) & v_{b}(t) & v_{c}(t)
\end{array}\right]^{T} \\
& \vec{i}_{a b c}(t)=\left[\begin{array}{lll}
i_{a}(t) & i_{b}(t) & i_{c}(t)
\end{array}\right]^{T}
\end{aligned}
$$

Figure 3.1 illustrates the instantaneous voltage vector components on the $a b c$ reference frame.

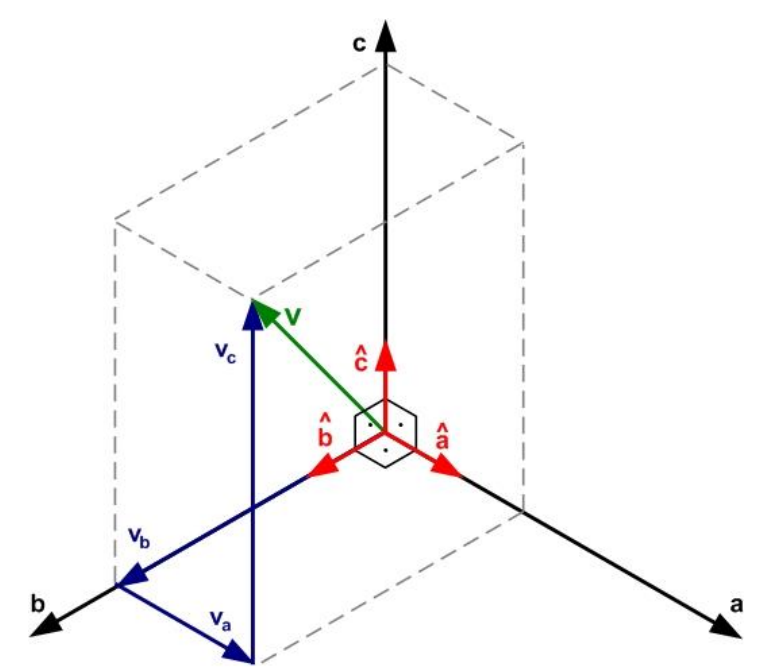

Figure 3.1: Three-dimensional abc reference frame

The voltage and the current vectors can be equivalently represented in other orthonormal coordinate basis, e.g. by employing Clarke's transformation; the basis is changed from the $a b c$ to the $\alpha \beta 0$ coordinate system, where the $p-q$ theory concepts are developed. 
The original and modified $p-q$ theories define instantaneous three-phase power calculations exclusively based on the $\alpha \beta 0$ transformation, which converts voltages and currents to the $\alpha \beta 0$ stationary reference frame. The zero sequence components are separated from the $\alpha \beta$ components by such transformation, which is convenient for instantaneous power flow analyses in ungrounded three-wire systems, as explained in Chapter 2, as such systems do not allow the flow of zero sequence current.

The $p-q$ theory states that it is impossible to produce constant zero sequence power, i.e., the average and oscillating terms related to zero sequence power coexist. The authors of the $p-q$ theory state that "zero-sequence components should be avoided in three-phase systems because these cannot produce three-phase constant power" [9]. As previously mentioned, the $p-q$ theory is attached to the stationary $\alpha \beta 0$ transformation, motivating investigations towards a novel transformation, which will be further introduced.

\section{2 - The mno Transformation}

The mno transformation relates voltages and currents in the $a b c$ to the mno coordinate systems, and vice-versa (inverse mno transformation). The transformation is based on the creation of the mno three-dimensional cartesian reference frame. It is a time domain basis that adjusts itself instantaneously according to a vector, referred to as the normal vector. The normal vector tracks the instantaneous three-phase measured voltage.

The complete mno transformation is expressed for the voltage vector by the following equation:

$$
\vec{v}_{m n o}^{d q}(t)=M_{d q} M_{m n o}(t) \vec{v}_{a b c}(t)
$$


where the matrix $M_{m n o}(t)$ converts the three instantaneous $a b c$ voltages $\vec{v}_{a b c}(t)$ into three instantaneous components in the mno reference frame; and the matrix $M_{d q}$ further decomposes each of these mno components into two direct-quadrature components, creating the six dimensional vector $\vec{v}_{m n o}^{d q}(t)$. This transformation produces the instantaneous voltage and current components required to evaluate the total three-phase power.

\subsection{1 - The mno Matrix}

The instantaneous voltage and current vectors can be expressed in the threedimensional mno cartesian coordinate system by:

$$
\begin{gathered}
\vec{v}_{\text {mпo }}(t)=v_{m}(t) \hat{m}+v_{n}(t) \hat{n}+v_{o}(t) \hat{o} \\
\vec{i}_{\text {тпo }}(t)=i_{m}(t) \hat{m}+i_{n}(t) \hat{n}+i_{o}(t) \hat{o}
\end{gathered}
$$

where $\hat{m}, \hat{n}, \hat{o}$ correspond to the unit vectors of the mno coordinate basis. In matrix notation, (3.6) and (3.7) are respectively represented by:

$$
\begin{gathered}
\vec{v}_{\text {mno }}(t)=\left[\begin{array}{lll}
v_{m}(t) & v_{n}(t) & v_{o}(t)
\end{array}\right]^{T} \\
\vec{i}_{\text {mno }}(t)=\left[\begin{array}{lll}
i_{m}(t) & i_{n}(t) & i_{o}(t)
\end{array}\right]^{T}
\end{gathered}
$$

The transformation of the three-phase voltage and three-phase current from $a b c$ to the mno coordinates are:

$$
\begin{gathered}
\vec{v}_{\text {mпo }}(t)=M_{\text {mпo }}(t) \vec{v}_{a b c}(t) \\
\vec{i}_{\text {mпo }}(t)=M_{\text {mno }}(t) \vec{i}_{a b c}(t)
\end{gathered}
$$

with the mno transformation matrix $M_{m n o}(t)$ being defined as: 


$$
M_{m n o}(t)=\left[\begin{array}{ccc}
\sin \theta_{a}(t) \cos \phi_{a}(t) & \sin \theta_{b}(t) \cos \phi_{b}(t) & \sin \theta_{c}(t) \cos \phi_{c}(t) \\
\sin \theta_{a}(t) \sin \phi_{a}(t) & \sin \theta_{b}(t) \sin \phi_{b}(t) & \sin \theta_{c}(t) \sin \phi_{c}(t) \\
\cos \theta_{a}(t) & \cos \theta_{b}(t) & \cos \theta_{c}(t)
\end{array}\right]
$$

and the inverse transformation which transfers the mno back to the $a b c$ coordinates being given by:

$$
\begin{gathered}
\vec{v}_{a b c}(t)=M_{m n o}^{-1}(t) \vec{v}_{m n o}(t) \\
\vec{i}_{a b c}(t)=M_{m n o}^{-1}(t) \vec{i}_{m n o}(t)
\end{gathered}
$$

where the inverse transformation matrix $M_{m n o}^{-1}(t)$ is defined as the transpose matrix of (3.12):

$$
\begin{gathered}
M_{m n o}^{-1}=M_{m n o}^{T} \\
M_{m n o}^{-1}(t)=\left[\begin{array}{ccc}
\sin \theta_{a}(t) \cos \phi_{a}(t) & \sin \theta_{a}(t) \sin \phi_{a}(t) & \cos \theta_{a}(t) \\
\sin \theta_{b}(t) \cos \phi_{b}(t) & \sin \theta_{b}(t) \sin \phi_{b}(t) & \cos \theta_{b}(t) \\
\sin \theta_{c}(t) \cos \phi_{c}(t) & \sin \theta_{c}(t) \sin \phi_{c}(t) & \cos \theta_{c}(t)
\end{array}\right]
\end{gathered}
$$

Matrices (3.12) and (3.16) are continuously adjusted by six time-dependent angles $\left(\theta_{a}\right.$, $\left.\theta_{b}, \theta_{c}, \phi_{a}, \phi_{b}, \phi_{c}\right)$, named mno angles, which are updated depending on the instantaneous normal vector (a vector reference for the mno transformation), as will be shown in sequence.

\subsection{2 - The Instantaneous Normal Vector}

The instantaneous normal vector is defined as the third vector of the set of three vectors that also includes the instantaneous voltage vector and the derivative of the 
instantaneous voltage vector (which is also the instantaneous tangent voltage vector), which all together form an orthonormal set (Figure 3.2a).

The instantaneous normal vector $\vec{n}_{a b c}(t)$ is obtained as shown in Figure $3.2 \mathrm{a}$ as the cross product of the instantaneous voltage vector $\vec{v}_{a b c}(t)$ and the instantaneous voltage tangent vector $d \vec{v}_{a b c}(t) / d t$ in $a b c$ coordinates:

$$
\vec{n}_{a b c}(t)=\vec{v}_{a b c}(t) \times \frac{d \vec{v}_{a b c}(t)}{d t}=\vec{n}_{a}(t) \hat{a}+\vec{n}_{b}(t) \hat{b}+\vec{n}_{c}(t) \hat{c}
$$

This vector is normalized to unit magnitude to yield the first mno basis vector $\hat{o}$ given by:

$$
\hat{o}(t)=\frac{\vec{n}_{v a b c}(t)}{\left|\vec{n}_{v a b c}(t)\right|}
$$

Figure $3.2 \mathrm{~b}$ illustrates the components of the normal vector for a section of the voltage locus curve, defined by the endpoints of the voltage vector $\vec{v}_{a b c}(t)$. The normal vector is orthonormal to the $m n$ plane, which is the plane defined by three immediately consecutives endpoints of the voltage vector $\vec{v}_{a b c}(t-\Delta t), \vec{v}_{a b c}(t)$, and $\vec{v}_{a b c}(t+\Delta t)$, as $\Delta t \rightarrow 0$. In a digital implementation, $\Delta t$ is a suitably small sampling time-step. The $m n$ plane also contains the instantaneous voltage vector and its tangent vector (Figure 3.2a). The instantaneous voltage tangent vector is calculated as:

$$
\frac{d \vec{v}_{a b c}(t)}{d t} \approx \frac{\left[\vec{v}_{a b c}(t+\Delta t)-\vec{v}_{a b c}(t)\right]}{\Delta t}
$$

which requires at least two immediately consecutive voltage samples of the measured voltage for the purpose of the derivative calculation. The origins of the normal vector 
$\vec{n}_{a b c}(t)$ and voltage vector $\vec{v}_{a b c}(t)$ coincide with the origin of the coordinate basis, also the center of the voltage locus curve (assuming no DC offset on the unbalanced phases).

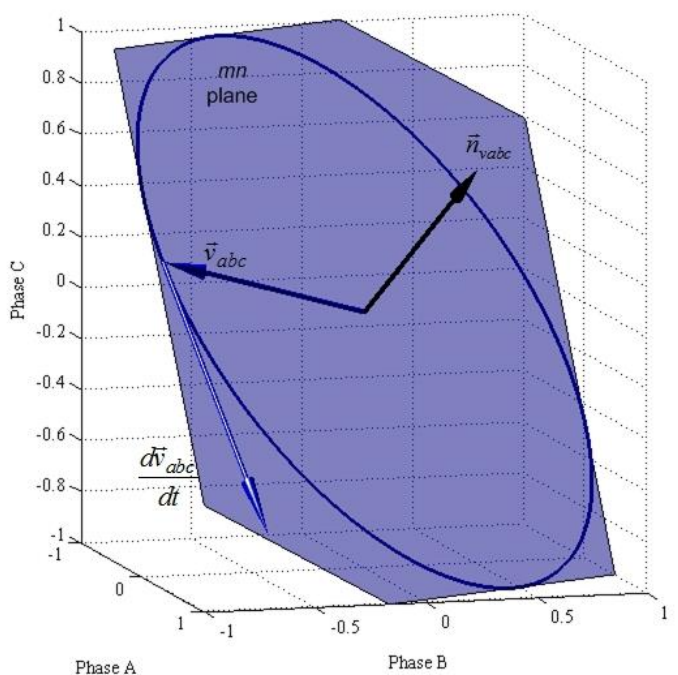

(a)

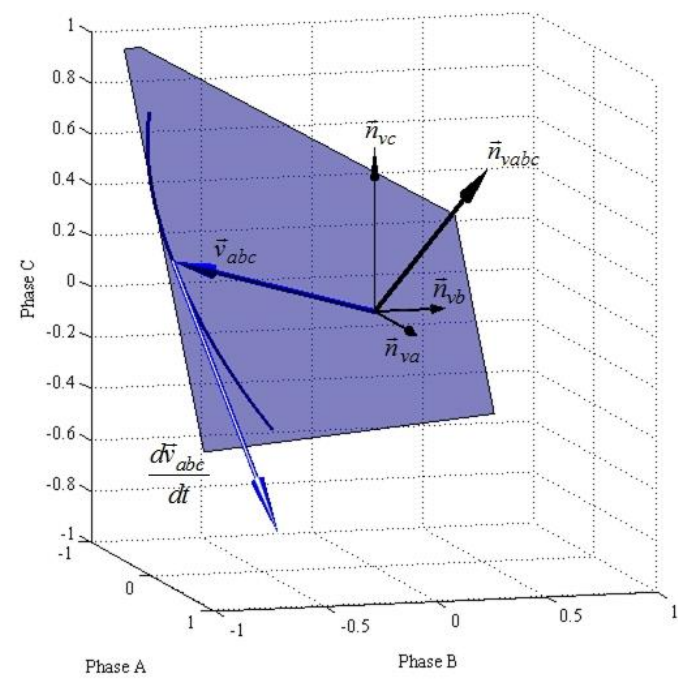

(b)

Figure 3.2: Instantaneous normal vector: (a) orthonormal set, (b) abc normal vector components

For a steady-state balanced three-phase voltage, the normal vector and the $m n$ plane are stationary, with the $m n$ plane being equivalent to the $\alpha \beta$ plane, defined by the $\alpha \beta 0$ transformation. For a fundamental component periodic unbalanced voltage, the $m n$ plane is still stationary, but no longer parallel to the $\alpha \beta$ plane. Furthermore, during transients, the normal vector and the $m n$ plane become time dependent, i.e. they may change their orientation every time-step.

If the three-phase voltage keeps changing dynamically, the mn plane and the normal vector will adjust themselves according to the instantaneous voltage conditions. The orientation of the instantaneous unit vector $\hat{o}$ (normalized normal vector) tracks the measured voltage, allowing the calculation of the mno angles, which can be subdivided in two sets of three angles: the mno pitch angles $\theta_{a}(t), \theta_{b}(t)$ and $\theta_{c}(t)$; and the mno yaw 
angles $\phi_{a}(t), \phi_{b}(t)$ and $\phi_{c}(t)$.

These angles establish relationships between the $a b c$ stationary coordinates and the adjustable mno reference frame. The mno angles represent an essential step to calculate each of the mno matrix elements and, consequently, to update the instantaneous mno voltage and mno current components. Figure 3.3 illustrates the $a b c$ and the mno reference coordinates, along with the projections used to evaluate the angles among the basis vectors, which will be explained in sequence.

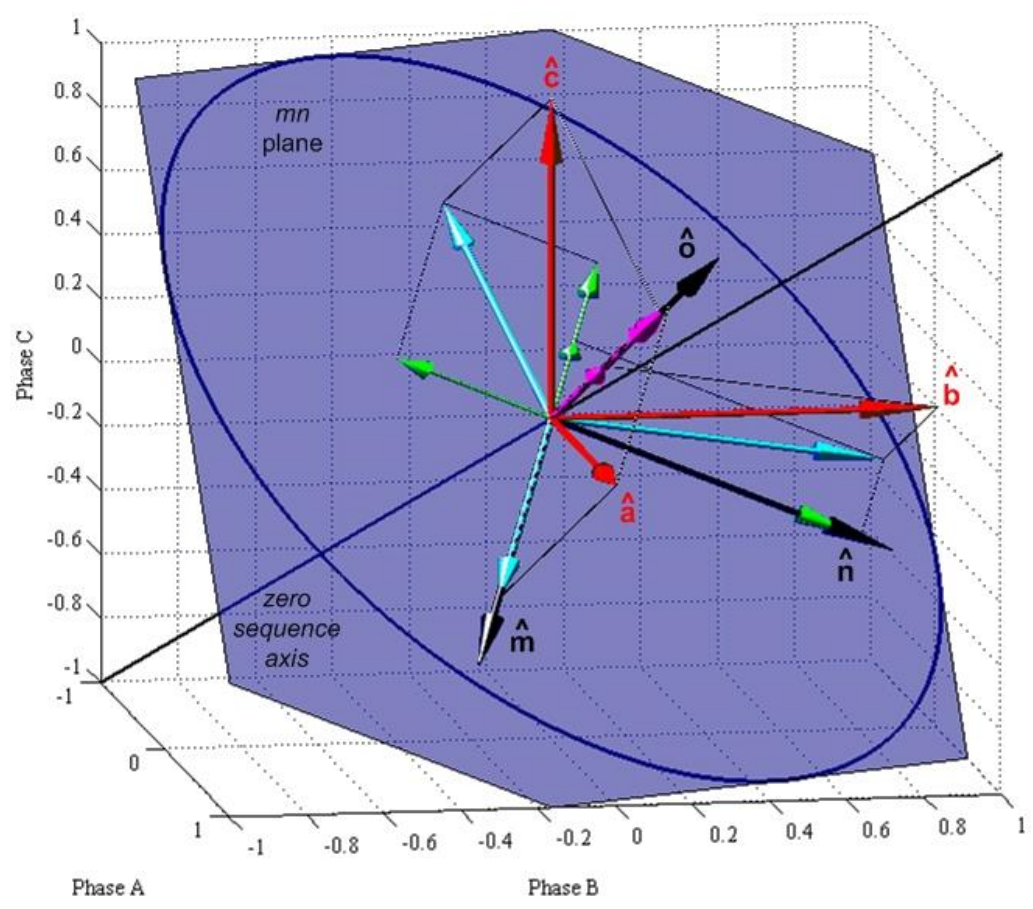

Figure 3.3: Creation of the mno basis (abc reference frame; mno reference frame; mn plane; projections; and zero sequence axis) 


\subsection{3 - The mno Angles}

The mno pitch angles $\theta_{a}(t), \theta_{b}(t)$ and $\theta_{c}(t)$ represent the angles between the directional unit vector $\hat{o}$ and the directional unit vectors of the original $a b c$ reference frame. They are calculated by using the dot product as:

$$
\begin{aligned}
& \theta_{a}(t)=\arccos (\hat{a} \cdot \hat{o}) \\
& \theta_{b}(t)=\arccos (\hat{b} \cdot \hat{o}) \\
& \theta_{c}(t)=\arccos (\hat{c} \cdot \hat{o})
\end{aligned}
$$

where, in a matrix representation, $\hat{a}=\left[\begin{array}{lll}1 & 0 & 0\end{array}\right]^{T}, \hat{b}=\left[\begin{array}{lll}0 & 1 & 0\end{array}\right]^{T}$ and $\hat{c}=\left[\begin{array}{lll}0 & 0 & 1\end{array}\right]^{T}$, corresponding to each one of the $a b c$ directional unit vectors. The mno pitch angles are illustrated on Figure 3.4.

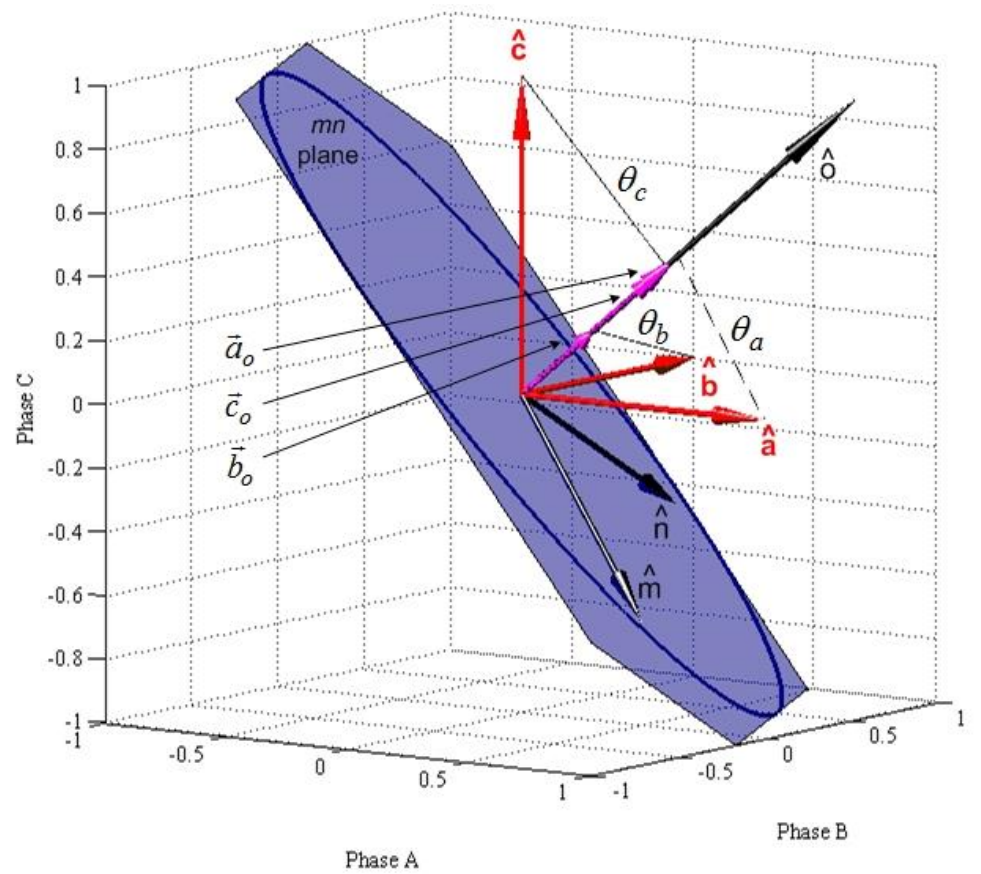

Figure 3.4: The mno pitch angles and the abc projections of the abc unit vectors onto the ô unit vector

This set of angles tracks the orientation of the $m n$ plane, allowing the evaluation of the vectors $\vec{a}_{o}(t), \vec{b}_{o}(t)$ and $\vec{c}_{o}(t)$, which are the respective projections of the unit vectors 
$\hat{a}, \hat{b}$ and $\hat{c}$ onto the unit vector $\hat{o}$ (Figure 3.4), defined using the dot product as:

$$
\begin{aligned}
& \vec{a}_{o}(t)=\cos \theta_{a} \cdot \hat{o} \\
& \vec{b}_{o}(t)=\cos \theta_{b} \cdot \hat{o} \\
& \vec{c}_{o}(t)=\cos \theta_{c} \cdot \hat{o}
\end{aligned}
$$

The magnitudes $\left|\vec{a}_{o}(t)\right|,\left|\vec{b}_{o}(t)\right|$ and $\left|\vec{c}_{o}(t)\right|$ of the vectors expressed by Equations (3.23), (3.24) and (3.25) are used at the third line of the mno matrix $M_{m n o}(t)$ to calculate the $a b c$ components contribution in the $\hat{o}$ direction.

The mno pitch angles can be applied to evaluate zero sequence content as a measurement of the system imbalance or to limit the excursion of controlled current signals in voltage sourced converter (Chapter 4). For instance, two fixed parallel planes orthogonal to the fixed zero sequence axis $\hat{z}=\left[\begin{array}{lll}1 & 1 & 1\end{array}\right]^{T}$ can be employed as allowed maximum and minimum limits for the zero sequence current excursion. Figure 3.5 illustrates this concept for an unbalanced three-phase voltage excursion, which relates to the actual instantaneous value of the zero sequence current, not the magnitude of the zero sequence current. For example, considering an unbalanced three-phase condition at fundamental frequency, the zero sequence current would be a fundamental frequency AC current; and the planes would show the limits of the positive and negative values for this zero sequence current. 


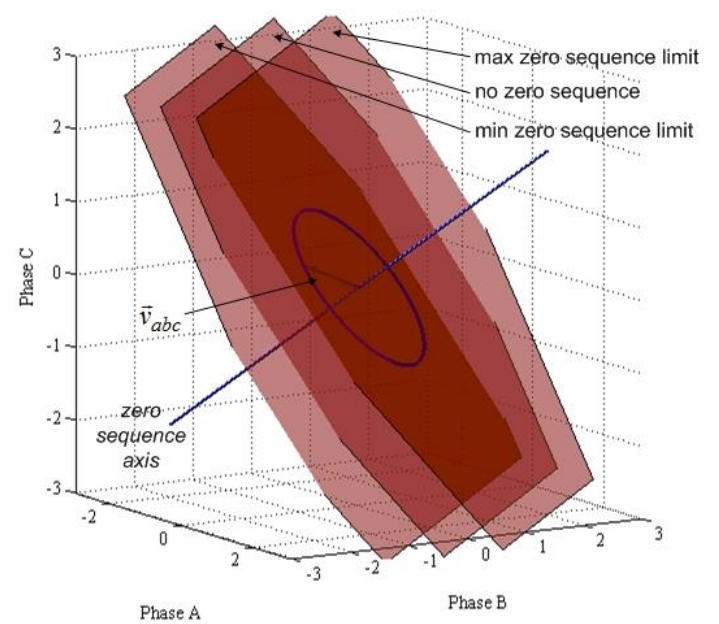

(a)

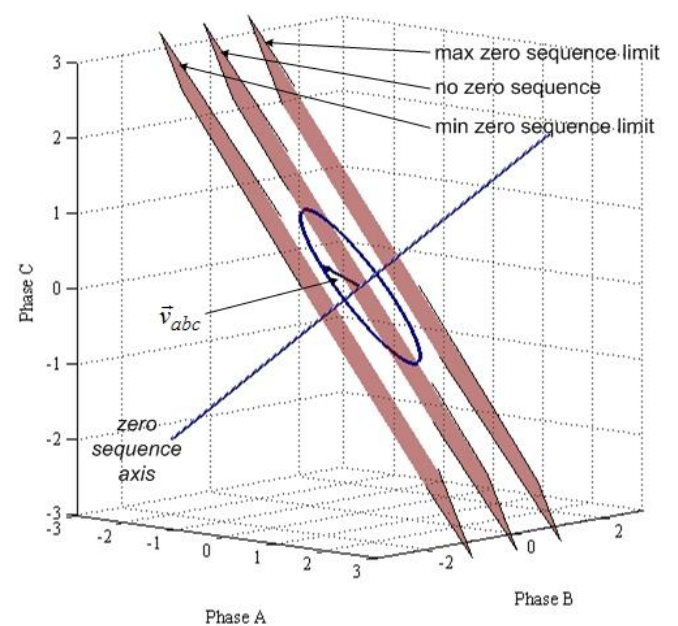

(b)

Figure 3.5: Fixed zero sequence planes: (a) view from outside the limits, (b) view from inside the limits

Besides the mno pitch angles, there are the mno yaw angles. The projections onto the $m n$ plane of the unit vectors $\hat{a}, \hat{b}$ and $\hat{c}$ (Figure 3.6) are respectively referred to as $\vec{a}_{m n}(t), \vec{b}_{m n}(t)$ and $\vec{c}_{m n}(t)$. The mno yaw angles $\phi_{a}(t), \phi_{b}(t)$ and $\phi_{c}(t)$ represent the angles made by these vectors $\vec{a}_{m n}(t), \vec{b}_{m n}(t), \vec{c}_{m n}(t)$ with the directional unit vector $\hat{m}$, which is the normalized projection of the unit vector $\hat{a}$ onto the $m n$ plane:

$$
\hat{m}=\frac{\vec{a}_{m n}(t)}{\left|\vec{a}_{m n}(t)\right|}
$$

and hence $\phi_{a}(t)=0$. The unit vector $\hat{m}$ is the second mno basis vector.

The calculation of the mno yaw angles can be subdivided in two steps. First, the vectors $\vec{a}_{m n}(t), \vec{b}_{m n}(t)$ and $\vec{c}_{m n}(t)$ are evaluated as a subtraction of two vectors as shown in Figure 3.6 and given by:

$$
\begin{aligned}
& \vec{a}_{m n}(t)=\hat{a}-\vec{a}_{o}(t) \\
& \vec{b}_{m n}(t)=\hat{b}-\vec{b}_{o}(t) \\
& \vec{c}_{m n}(t)=\hat{c}-\vec{c}_{o}(t)
\end{aligned}
$$


where $\vec{a}_{o}(t), \vec{b}_{o}(t)$ and $\vec{c}_{o}(t)$ can be respectively evaluated by Equations (3.23), (3.24) and (3.25).

Secondly, the angles between $\vec{a}_{m n}(t)$ and $\vec{b}_{m n}(t)$, as well as between $\vec{a}_{m n}(t)$ and $\vec{c}_{m n}(t)$ are calculated (Figure 3.6), with the angle $\phi_{a}(t)$ set to be zero by definition, as a locked reference between $\vec{a}_{m n}(t)$ and the basis vector $\hat{m}$ :

$$
\begin{gathered}
\phi_{a}(t)=0 \\
\phi_{b}(t)=\arccos \left(\frac{\vec{a}_{m n}(t) \cdot \vec{b}_{m n}(t)}{\left|\vec{a}_{m n}(t)\right|\left|\vec{b}_{m n}(t)\right|}\right) \\
\phi_{c}(t)=\arccos \left(\frac{\vec{a}_{m n}(t) \cdot \vec{c}_{m n}(t)}{\left|\vec{a}_{m n}(t)\right| \vec{c}_{m n}(t) \mid}\right)
\end{gathered}
$$

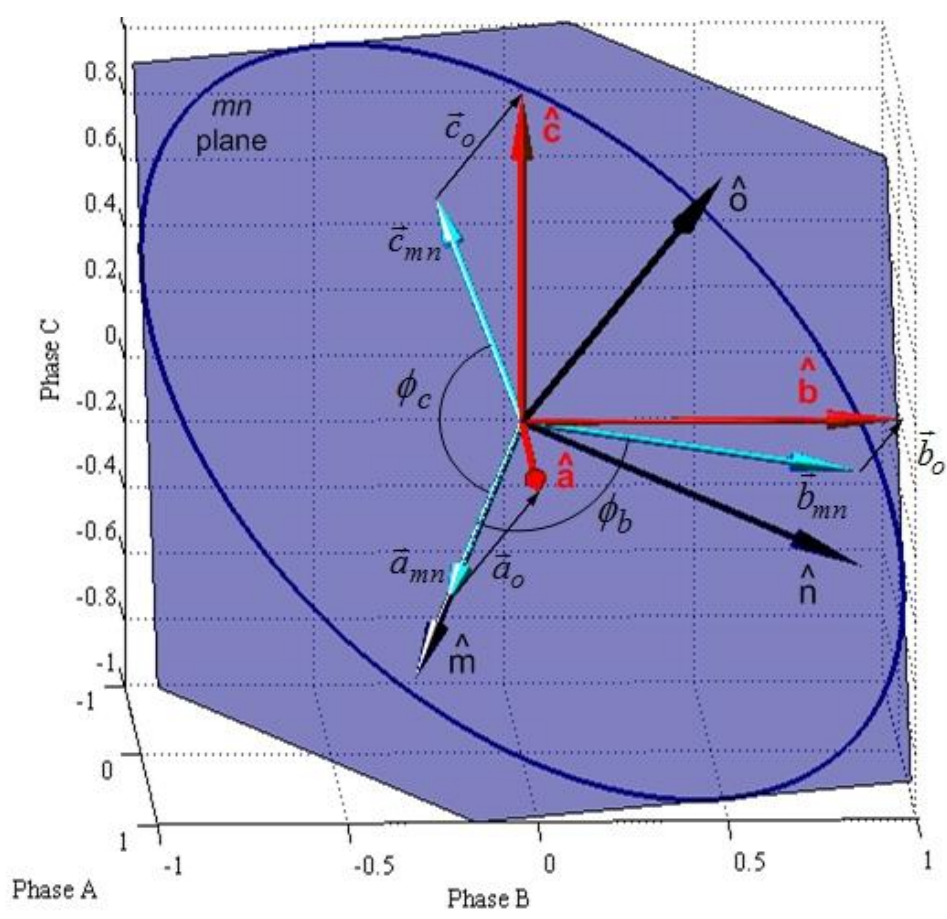

Figure 3.6: The mno yaw angles and the abc projections of the abc unit vectors onto the mn plane 
Note that the direction of the third and last unit basis vector $\hat{n}$ of the adaptive mno basis is orthonormal to the other basis vectors $\hat{o}$ and $\hat{m}$, being calculated as:

$$
\hat{n}(t)=\hat{o}(t) \times \hat{m}(t)
$$

Figure 3.7 summarizes the main steps necessary to calculate the mno angles of an arbitrary three-phase voltage at a specific instant, as well as the mno basis vectors.

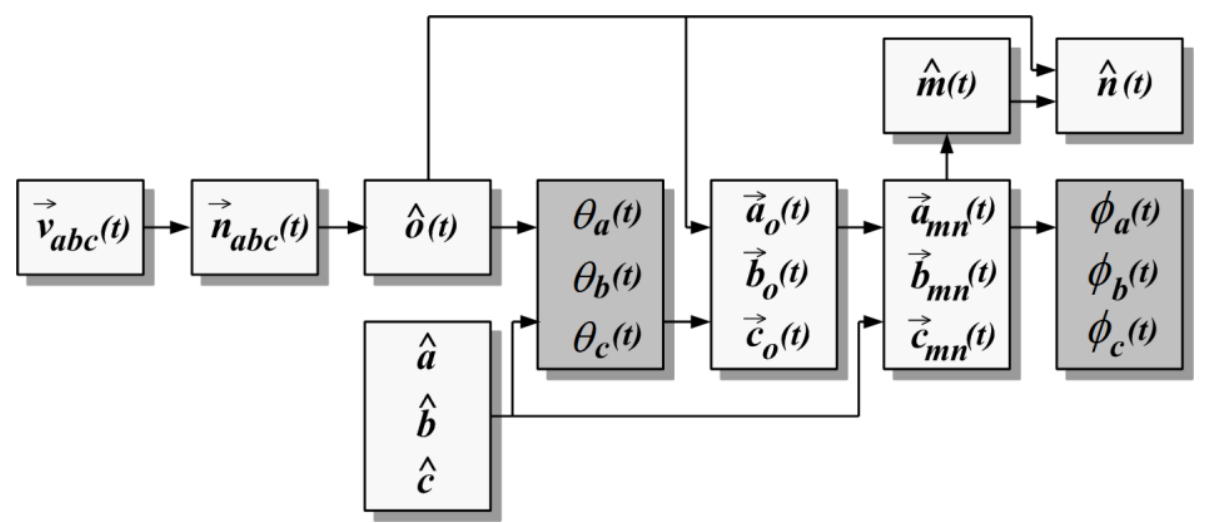

Figure 3.7: Main steps to evaluate the mno angles

The angles $\phi_{b}(t)$ and $\phi_{c}(t)$ can be used to calculate the projections of the vectors

$\vec{a}_{m n}(t), \vec{b}_{m n}(t)$ and $\vec{c}_{m n}(t)$ in the $\hat{m}$ and $\hat{n}$ directions (Figure 3.8) through the following expressions:

$$
\begin{aligned}
& \vec{b}_{m}(t)=\vec{b}_{m n}(t) \cos \phi_{b}(t) \\
& \vec{c}_{m}(t)=\vec{c}_{m n}(t) \cos \phi_{c}(t) \\
& \vec{b}_{n}(t)=\vec{b}_{m n}(t) \sin \phi_{b}(t) \\
& \vec{c}_{n}(t)=-\vec{c}_{m n}(t) \sin \phi_{c}(t)
\end{aligned}
$$




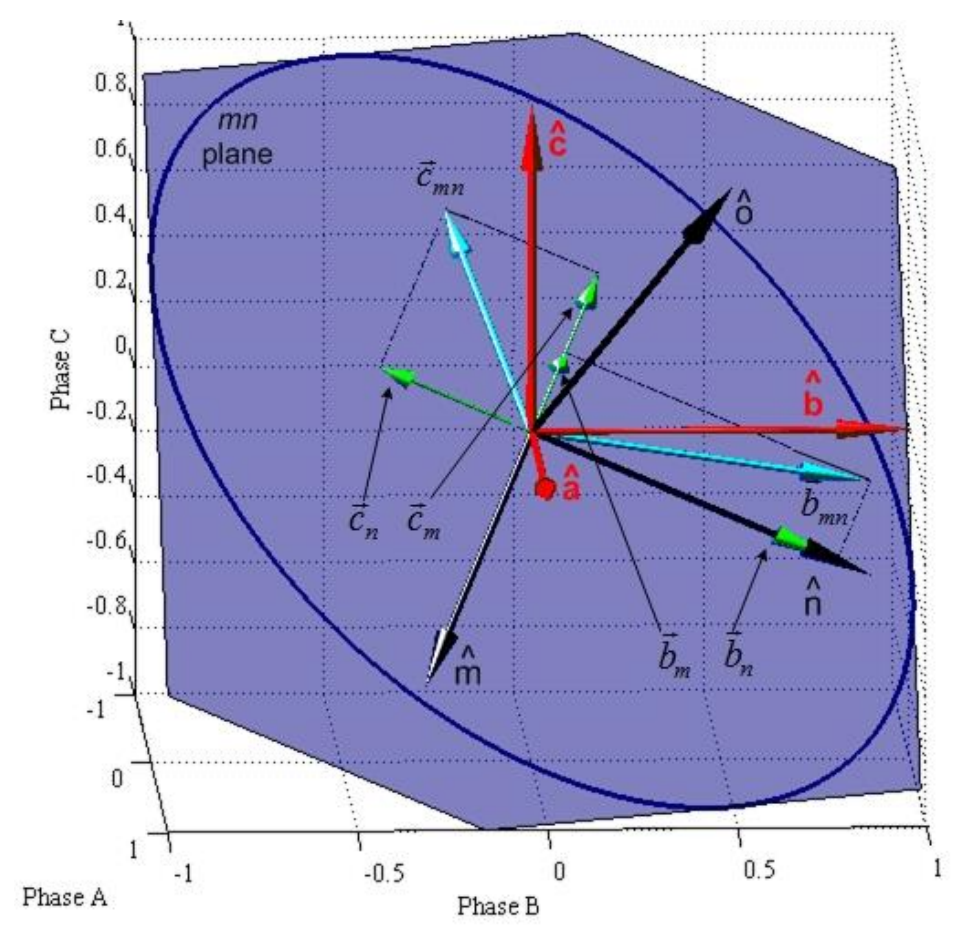

Figure 3.8: Projections of the abc versors onto the $\hat{m}$ and $\hat{n}$ directions

Due to the positive and negative values that the sine function can assume and the positive direction of the $\hat{n}$ axis, a negative sign on Equation (3.37) must exist to represent the accurate direction in the $\hat{n}$ axis.

The magnitudes $\left|\vec{b}_{m}(t)\right|,\left|\vec{c}_{m}(t)\right|,\left|\vec{b}_{n}(t)\right|$ and $\left|\vec{c}_{n}(t)\right|$ related to the vectors expressed by Equations (3.34) to (3.37) are used at the first and second lines of the mno matrix $M_{m n o}(t)$ to calculate the $a b c$ components contribution in the $\hat{m}$ and $\hat{n}$ directions.

Finally, as an illustrative example, all the projections of the $a b c$ components onto the mno reference frame are shown in Figure 3.9, highlighting the output components of the mno matrix transformation without the $a b c$ and mno basis vectors. 


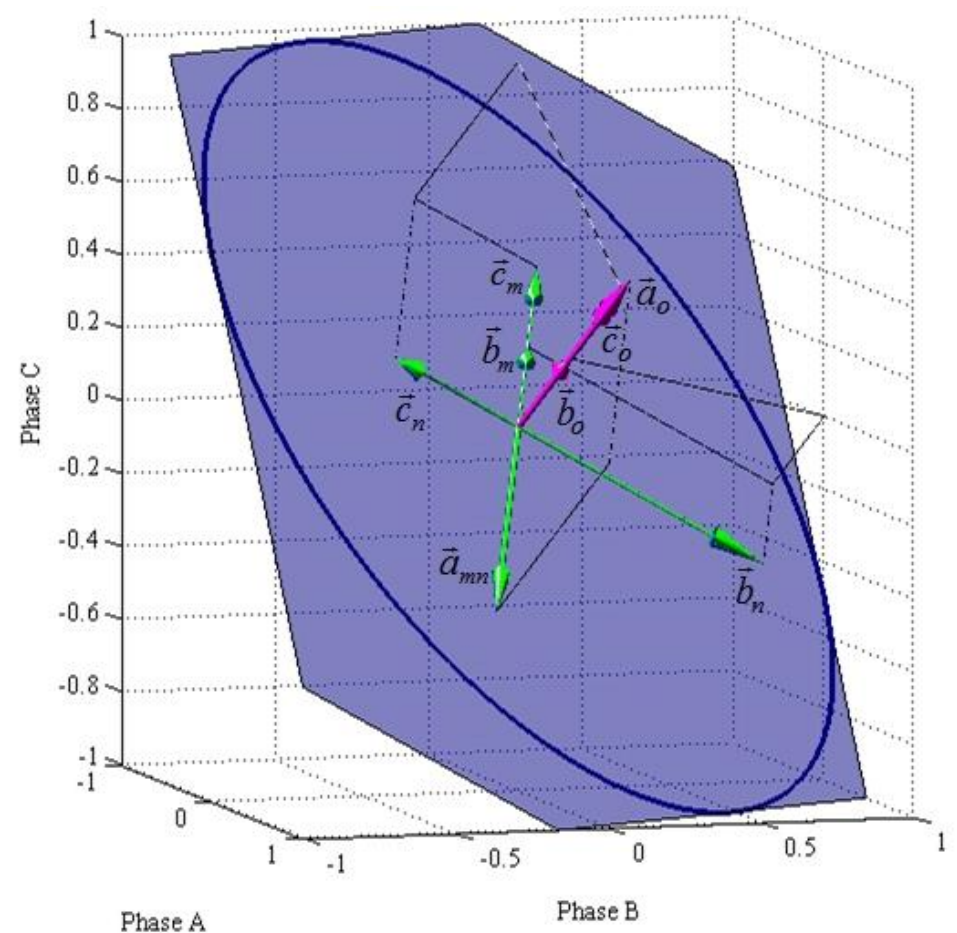

Figure 3.9: Projections of the abc unit vectors onto the $\hat{m}, \hat{n}$ and $\hat{o}$ directions

\subsection{4 - The $a b 0$ Transformation as a Special Case of the mno \\ Transformation}

The $\alpha \beta 0$ transformation becomes a particular case of the mno transformation for balanced conditions. The calculation of the mno angles for a balanced three-phase voltage using the procedure presented in the Section 3.2.3 leads to the following numerical results for the mno pitch angles:

$$
\left\{\begin{array}{l}
\theta_{a}(t)=54.73^{\circ} \\
\theta_{b}(t)=54.73^{\circ} \\
\theta_{c}(t)=54.73^{\circ}
\end{array}\right.
$$

and to the following values for the mno yaw angles: 


$$
\left\{\begin{array}{l}
\phi_{a}(t)=0 \\
\phi_{b}(t)=120^{\circ} \\
\phi_{c}(t)=-120^{\circ}
\end{array}\right.
$$

Substituting these angles in the mno matrix (3.12) gives:

$$
M_{\text {mno }}(t)=\left[\begin{array}{ccc}
\sin \left(54.73^{\circ}\right) \cos (0) & \sin \left(54.73^{\circ}\right) \cos \left(120^{\circ}\right) & \sin \left(54.73^{\circ}\right) \cos \left(-120^{\circ}\right) \\
\sin \left(54.73^{\circ}\right) \sin (0) & \sin \left(54.73^{\circ}\right) \sin \left(120^{\circ}\right) & \sin \left(54.73^{\circ}\right) \sin \left(-120^{\circ}\right) \\
\cos \left(54.73^{\circ}\right) & \cos \left(54.73^{\circ}\right) & \cos \left(54.73^{\circ}\right)
\end{array}\right]
$$

which, after simplifications, leads to the $\alpha \beta 0$ transformation $C_{\alpha \beta 0}$ (Clarke's transformation) from the mno matrix:

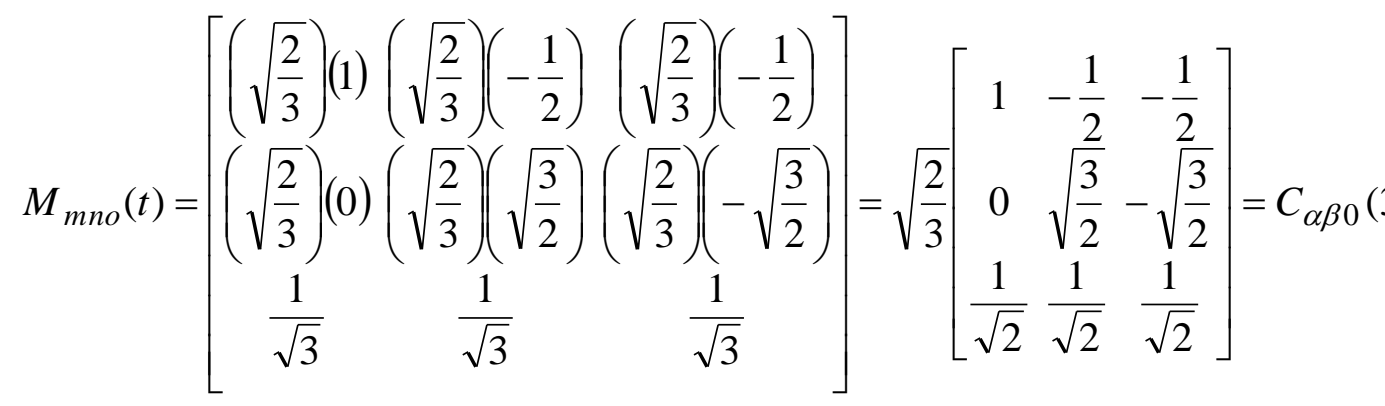

The elements of the $\alpha \beta 0$ transformation are time invariant. In contrast, the adaptive time dependent mno transformation adjusts its elements to different dynamic conditions.

As stated earlier, the instantaneous voltage and current vectors can be expressed in a three-phase $\alpha \beta 0$ cartesian coordinate system by using the Clarke transformation as:

$$
\begin{gathered}
\vec{v}_{\alpha \beta 0}(t)=v_{\alpha}(t) \hat{\alpha}+v_{\beta}(t) \hat{\beta}+v_{0}(t) \hat{0} \\
\vec{i}_{\alpha \beta 0}(t)=i_{\alpha}(t) \hat{\alpha}+i_{\beta}(t) \hat{\beta}+i_{0}(t) \hat{0}
\end{gathered}
$$

where $\hat{\alpha}, \hat{\beta}$ and $\hat{0}$ are the unit vectors of the $\alpha \beta 0$ cartesian system. Figure $3.10 \mathrm{a}$ shows the three three-dimensional cartesian coordinate systems for a balanced scenario: the $a b c$ 
reference frame; the mno reference frame; and the $\alpha \beta 0$ reference frame, which coincides to the mno reference frame for this condition.

Figure $3.10 \mathrm{~b}$ shows exactly the same scenario of Figure 3.10a, except that it is from a different point of view, i.e. from the top of the zero sequence axis, where the threedimensional representation of the unit vectors $\hat{a}, \hat{b}$ and $\hat{c}$ are shown similar as the traditional planar representation of the symmetrical components used in phasors diagrams, proposed by Fortescue [32].

The zero sequence axis is reduced to a single point on the $\alpha \beta$ plane. This plane also contains the $\hat{\alpha}$ and $\hat{\beta}$ directional unit vectors, able to represent positive and negative sequence values.

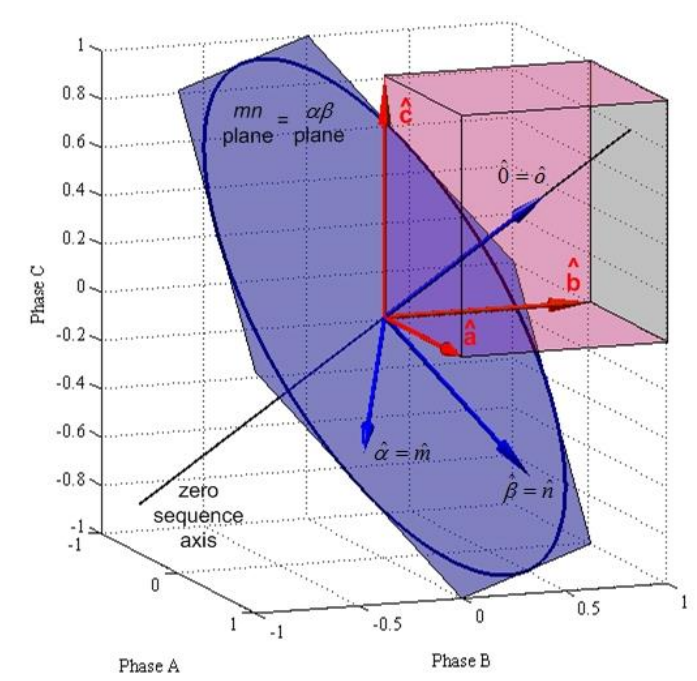

(a)

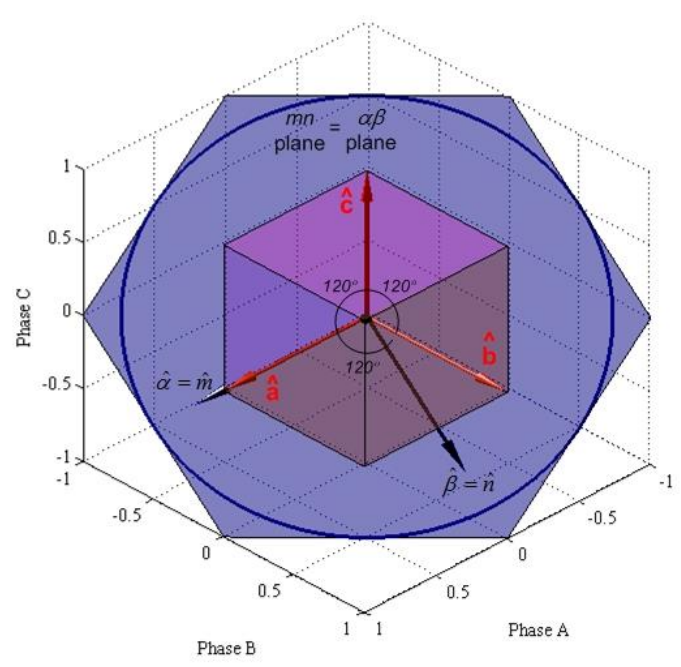

(b)

Figure 3.10: Clarke's transformation represented in the abc three-dimensional coordinate system:

(a) three-dimensional view, (b) view from the top of the zero sequence axis (two-dimensional view)

The representation proposed by Fortescue in the frequency domain (Figure 2.3, Chapter 2) includes zero sequence components in a planar representation (along with the 
positive and negative sequences), as a projection "shade" on a plane of a "tilted" threedimensional vector, like the vector $\hat{o}$ proposed by the mno transformation.

When the system is unbalanced, the $\hat{o}$ vector of the mno transformation is no longer aligned with the zero sequence axis, and its projection on the fixed $\alpha \beta$ plane (balanced plane) is represented by a vector, not a single point. The reader is reminded of the Allegory of the Cave analogy [1] sustained in the introduction (Chapter 1).

The next section introduces the second part of the mno transformation, a directquadrature decomposition of the mno components.

\subsection{5 - The Direct and Quadrature mno Matrix}

Each of the three instantaneous mno components obtained from the matrix $M_{m n o}(t)$ are decomposed into two direct-quadrature components by the following matrix:

$$
M_{d q}=\frac{1}{2}\left[\begin{array}{ccc}
1+j & 0 & 0 \\
1-j & 0 & 0 \\
0 & 1+j & 0 \\
0 & 1-j & 0 \\
0 & 0 & 1+j \\
0 & 0 & 1-j
\end{array}\right]
$$

where the operator $j=\sqrt{-1}$.

The matrix (3.44) is applied to the mno voltage $\vec{v}_{m n o}(t)$ and mno current $\vec{i}_{m n o}(t)$, generating the respective $d q$ mno voltage and current components through the transformation:

$$
\vec{v}_{m n o}^{d q}(t)=M_{d q} \vec{v}_{m n o}(t)
$$




$$
\vec{i}_{m n o}^{d q}(t)=M_{d q} \vec{i}_{m n o}(t)
$$

which creates $d q$ mno components for the voltage and $d q$ mno components for the current being defined as:

$$
\begin{aligned}
& \vec{v}_{m n o}^{d q}(t)=\left[\begin{array}{llllll}
v_{m}^{d}(t) & v_{m}^{q}(t) & v_{n}^{d}(t) & v_{n}^{q}(t) & v_{o}^{d}(t) & v_{o}^{q}(t)
\end{array}\right]^{T} \\
& \vec{i}_{m n o}^{d q}(t)=\left[i_{m}^{d}(t) i_{m}^{q}(t) i_{n}^{d}(t) i_{n}^{q}(t) i_{o}^{d}(t) i_{o}^{q}(t)\right]^{T}
\end{aligned}
$$

Equations (3.45) and (3.46) generate two extra $d q$ components for each one of the three components of the mno vectors $\vec{v}_{\text {mno }}(t)$ and $\vec{i}_{\text {mno }}(t)$, totalizing six mno components for the voltage $\vec{v}_{m n o}^{d q}(t)$ and six mno symmetrical components for the current $\vec{i}_{m n o}^{d q}(t)$.

The inverse transformation which provides the mno components in terms of the corresponding $d q$ mno components are calculated for the voltage and the current as:

$$
\begin{aligned}
& \left\{\begin{array}{l}
v_{m}=v_{m}^{d}+v_{m}^{q} \\
v_{n}=v_{n}^{d}+v_{n}^{q} \\
v_{o}=v_{o}^{d}+v_{o}^{q}
\end{array}\right. \\
& \left\{\begin{array}{l}
i_{m}=i_{m}^{d}+i_{m}^{q} \\
i_{n}=i_{n}^{d}+i_{n}^{q} \\
i_{o}=i_{o}^{d}+i_{o}^{q}
\end{array}\right.
\end{aligned}
$$

\subsection{7 - Numerical Example}

Consider the following unbalanced voltage and current with positive, negative and zero sequence content (per unit values and angular frequency $\omega=2 \pi 50 \mathrm{rad} / \mathrm{s}$ ): 


$$
\begin{aligned}
& \left\{\begin{array}{l}
v_{a}(t)=0.8 \cos (\omega t-\pi / 6) \\
v_{b}(t)=\cos (\omega t-2 \pi / 3) \\
v_{c}(t)=\cos (\omega t+2 \pi / 3)
\end{array}\right. \\
& \left\{\begin{array}{l}
i_{a}(t)=1.5 \cos (\omega t) \\
i_{b}(t)=\cos (\omega t-7 \pi / 9) \\
i_{c}(t)=\cos (\omega t+\pi)
\end{array}\right.
\end{aligned}
$$

The matrix $M_{m n o}(t)$ is evaluated based on the voltage. Figure 3.11 illustrates two cycles of the above waveforms in the $a b c$ and mno coordinates, as well as the $\alpha \beta 0$ components for comparison. Note that due to the particular choice of the references of the mno coordinate system previously introduced, the $\hat{o}$ component for the mno voltage $\vec{v}_{m n o}$ is zero.

Figure 3.12 shows the excursion of the instantaneous voltage and current vectors in the $a b c$ and the mno three-dimensional reference frames for one cycle. The instantaneous voltage and current vectors allow the calculation of the instantaneous power, as it will be demonstrated by the mno instantaneous power equations presented in the next section. 


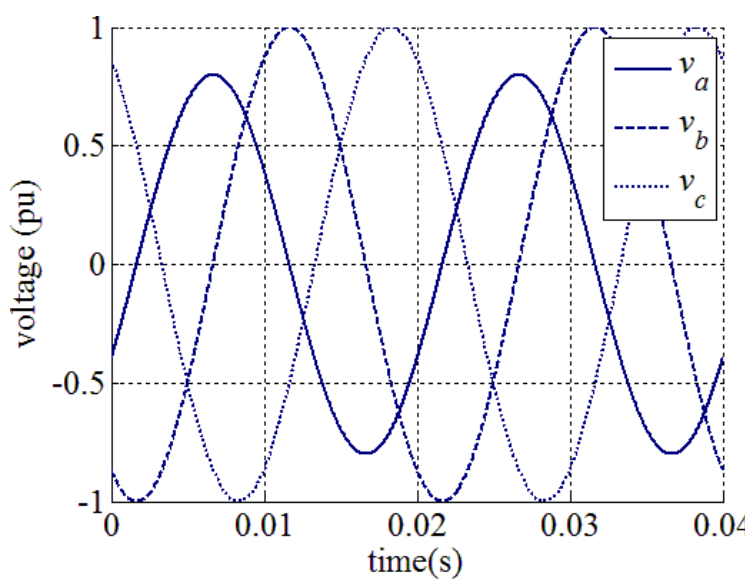

a)

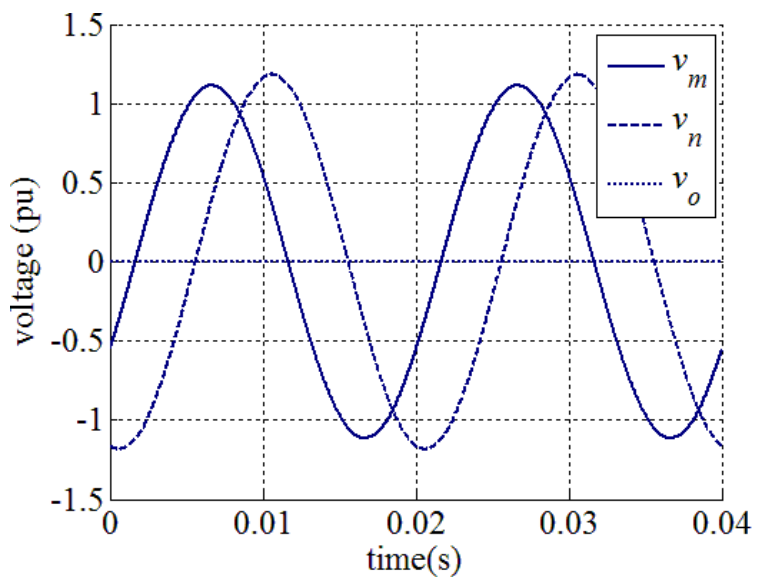

(c)

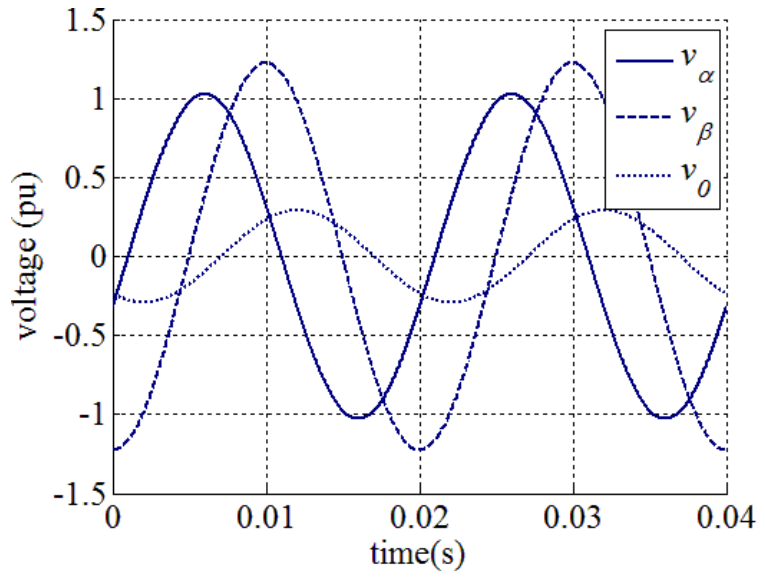

(e)

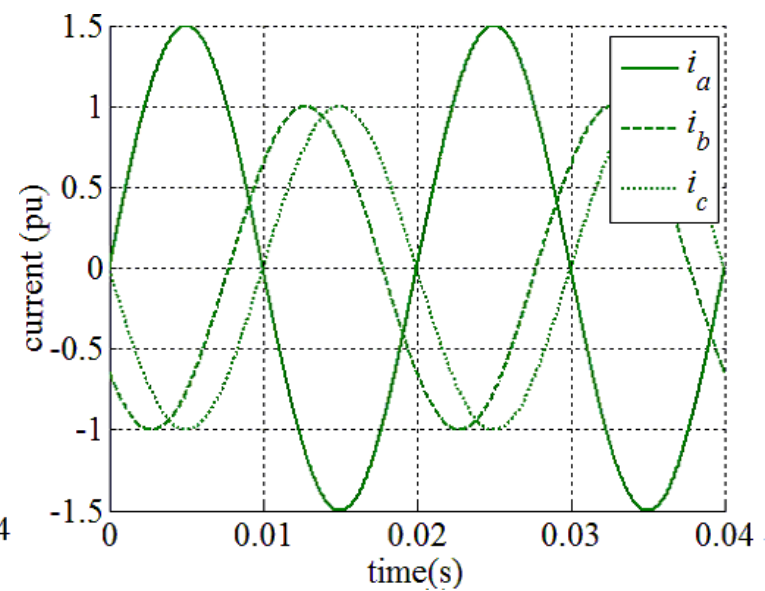

(b)

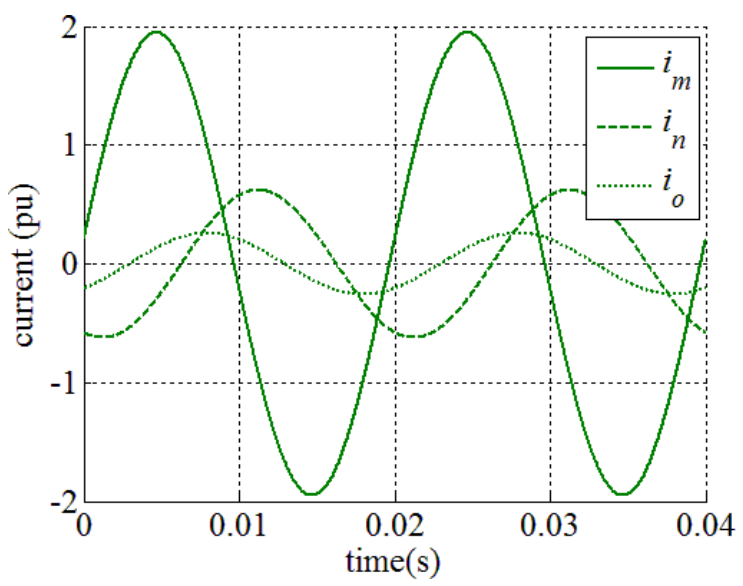

(d)

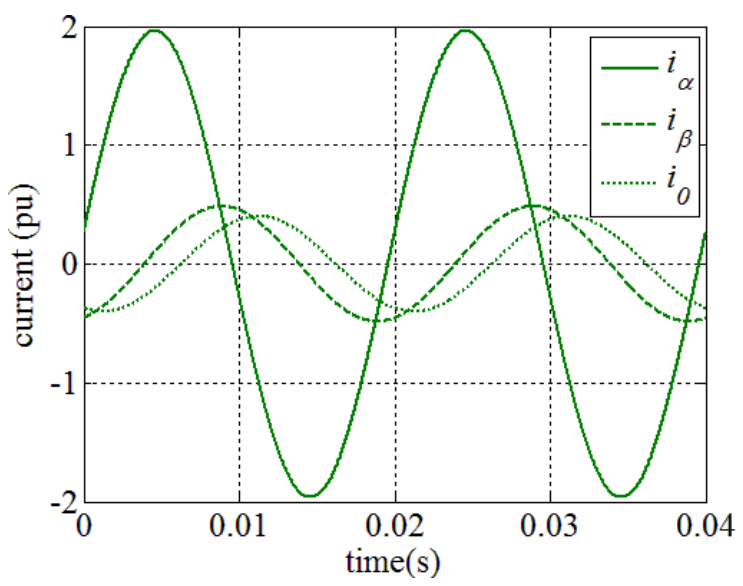

$(f)$

Figure 3.11: The mno and $\alpha \beta 0$ components: (a) abc voltage, (b) abc current, (c) mno voltage, (d) mno current, (e) $\alpha \beta 0$ voltage, $(f) \alpha \beta 0$ current 


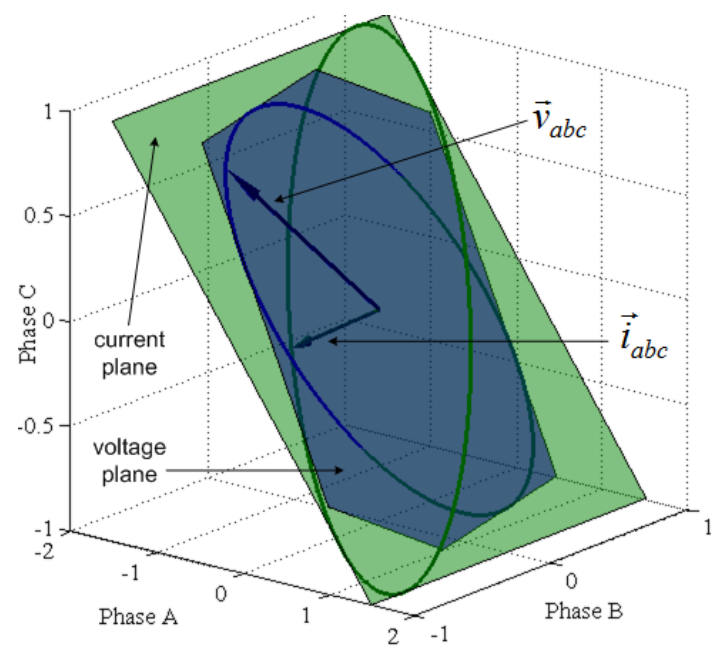

(a)

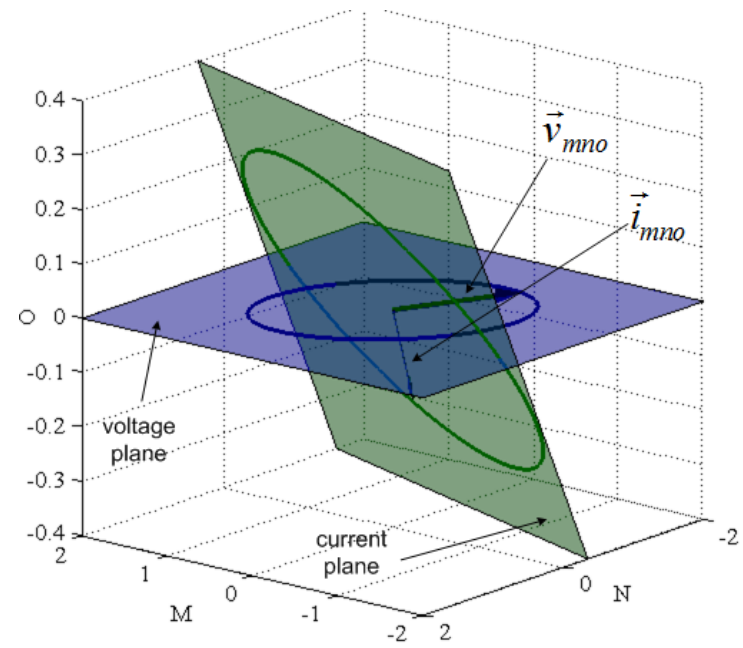

(b)

Figure 3.12: Voltage and current signals loci in the abc and mno time domain three-dimensional reference frame: (a) abc voltage and abc current, (c) mno voltage and mno current

After generating the instantaneous mno components, the $d q$ mno matrix (3.44) is applied. Figure 3.13 illustrates the voltage and current signals with the $\pi / 2 \mathrm{rad}$ phase shift between the $d q$ components. 


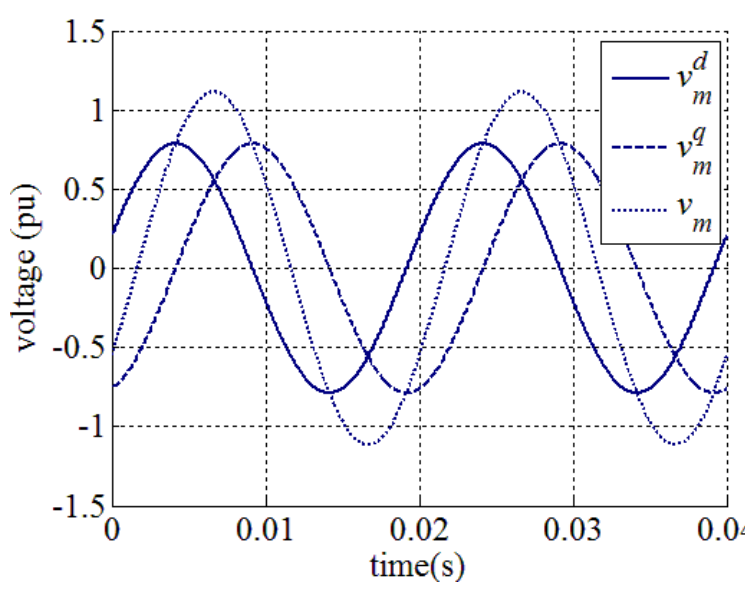

(a)

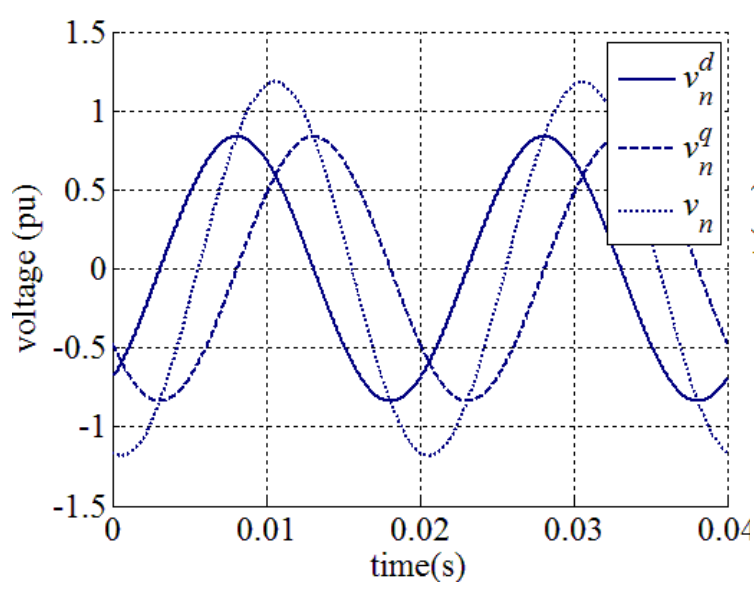

(c)

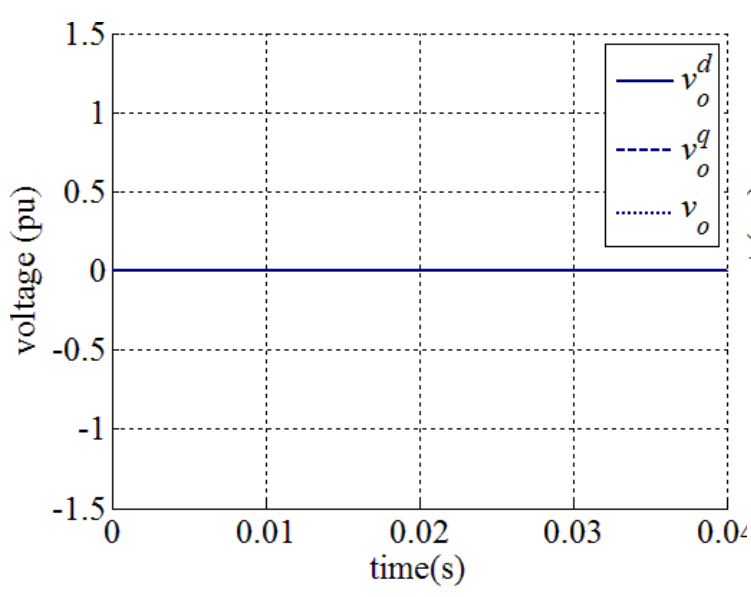

(e)

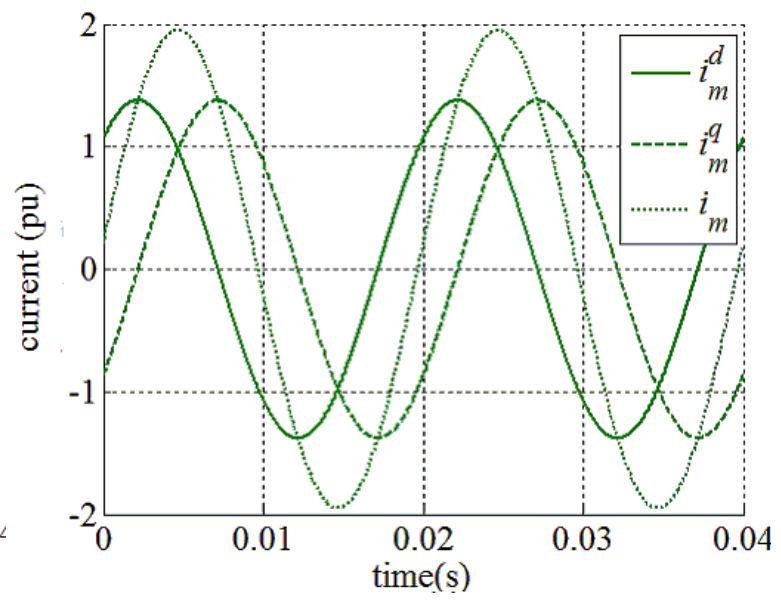

(b)

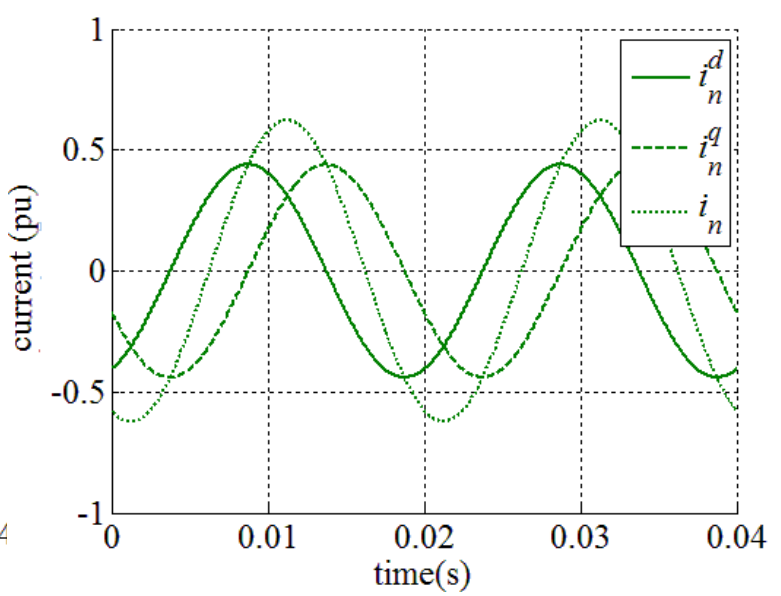

(d)

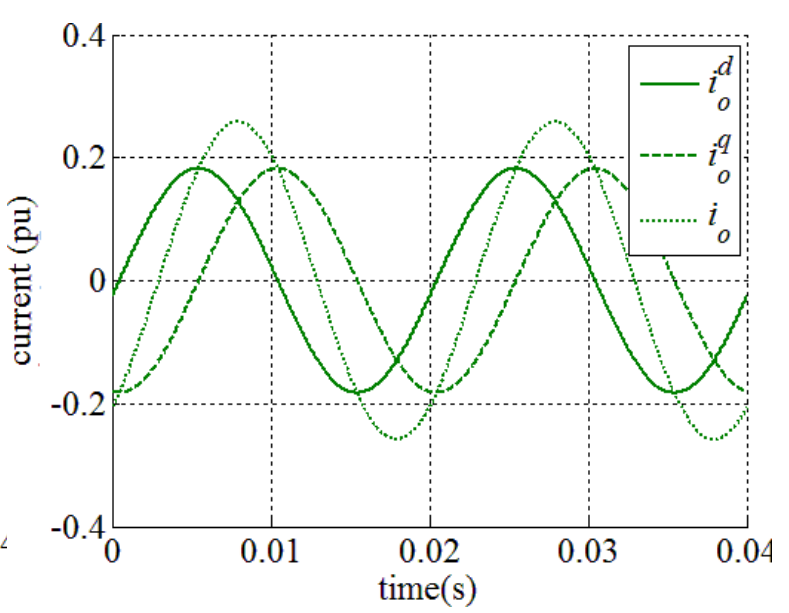

(f)

Figure 3.13: The dq mno components: (a) dq $m$ voltage, (b) $d q m$ current, (c) $d q n$ voltage, (d) $d q n$ current, (e) dq o voltage, $(f)$ dq o current 


\section{3 - The mno Instantaneous Power Components}

This section presents a new method developed to calculate the three-phase instantaneous power. The technique is based on the instantaneous mno voltage and mno current components generated by the mno transformation. It allows an independent calculation of constant and oscillating terms which contribute to the total instantaneous active and reactive power on general three-phase transmission topologies.

The technique stands out during unbalanced AC conditions with three independent voltages and three independent currents, i.e. with positive, negative and zero sequence power flowing through the system. Such feature along with the development of new power control strategies emerged as a pursued research outcome which allows a constant power flow to be established in grid connected voltage sourced converters under unbalanced conditions.

The equations presented for the mno power theory are an extension of the $p$ - $q$ theory concepts introduced in the Chapter 2 , which are based on the stationary $\alpha \beta 0$ transformation. The instantaneous active and reactive powers are evaluated, respectively, by the dot and the cross product of the instantaneous mno voltage and the instantaneous mno current vectors. In this approach, the active power comes out as a scalar and the reactive power as a vector:

$$
\begin{gathered}
p(t)=\vec{v}_{m n o}(t) \cdot \vec{i}_{m n o}(t) \\
\vec{q}(t)=\vec{v}_{m n o}(t) \times \vec{i}_{m n o}(t)
\end{gathered}
$$

Accordingly, the instantaneous active power is obtained as:

$$
p(t)=p_{m}(t)+p_{n}(t)+p_{o}(t)=v_{m}(t) i_{m}(t)+v_{n}(t) i_{n}(t)+v_{o}(t) i_{o}(t)
$$


and the instantaneous reactive power magnitude is given by:

$$
|\vec{q}(t)|=\left|q_{m}(t) \hat{m}+q_{n}(t) \hat{n}+q_{o}(t) \hat{o}\right|=\sqrt{\left[q_{m}(t)\right]^{2}+\left[q_{n}(t)\right]^{2}+\left[q_{o}(t)\right]^{2}}
$$

where

$$
\begin{aligned}
& q_{m}(t)=\left|\begin{array}{ll}
v_{n}(t) & v_{o}(t) \\
i_{n}(t) & i_{o}(t)
\end{array}\right|=\left[v_{n}(t) i_{o}(t)-v_{o}(t) i_{n}(t)\right] \\
& q_{n}(t)=\left|\begin{array}{ll}
v_{o}(t) & v_{m}(t) \\
i_{o}(t) & i_{m}(t)
\end{array}\right|=\left[v_{o}(t) i_{m}(t)-v_{m}(t) i_{o}(t)\right] \\
& q_{o}(t)=\left|\begin{array}{ll}
v_{m}(t) & v_{n}(t) \\
i_{m}(t) & i_{n}(t)
\end{array}\right|=\left[v_{m}(t) i_{n}(t)-v_{n}(t) i_{m}(t)\right]
\end{aligned}
$$

Figure 3.14 illustrates power waveforms related to Equations (3.55) to (3.59) for the unbalanced voltage and current condition (3.51).

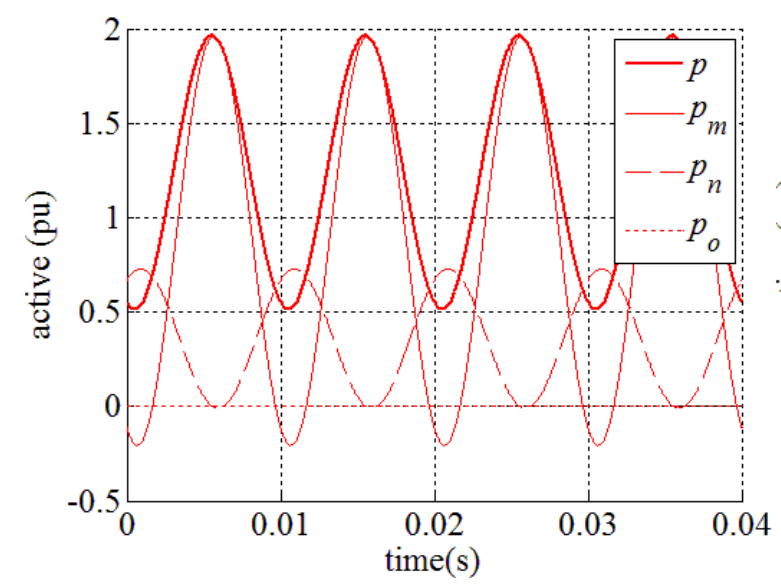

(a)

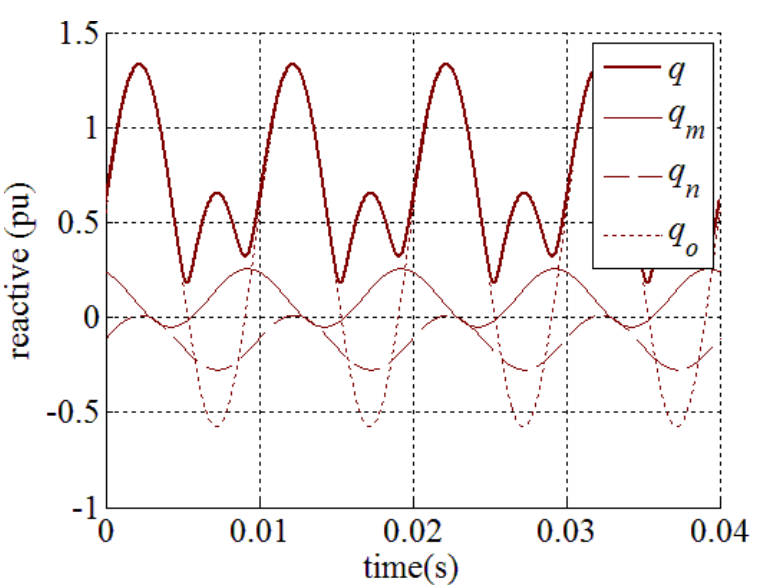

(b)

Figure 3.14: Instantaneous power: (a) active power, (b) reactive power

The waveforms for $p$ and $|\vec{q}|$ perfectly agree with the total active and reactive instantaneous power calculations of the modified $p$ - $q$ theory [9], except that the mno power components $p_{m}, p_{n}, p_{o}, q_{m}, q_{n}$ and $q_{o}$ are not consistent to the respective $\alpha \beta O$ power components $p_{\alpha}, p_{\beta}, p_{0}, q_{\alpha}, q_{\beta}$ and $q_{0}$, defined as: 


$$
\begin{gathered}
p(t)=p_{\alpha}(t)+p_{\beta}(t)+p_{0}(t)=v_{\alpha}(t) i_{\alpha}(t)+v_{\beta}(t) i_{\beta}(t)+v_{0}(t) i_{0}(t) \\
|\vec{q}(t)|=\left|q_{\alpha}(t) \hat{\alpha}+q_{\beta}(t) \hat{\beta}+q_{0}(t) \hat{0}\right|=\sqrt{\left[q_{\alpha}(t)\right]^{2}+\left[q_{\beta}(t)\right]^{2}+\left[q_{0}(t)\right]^{2}}
\end{gathered}
$$

Substituting the $d q$ mno components (3.49) and (3.50) into Equations (3.55), (3.57), (3.58) and (3.59) enables the decomposition into the following form:

$$
\begin{gathered}
p(t)=\bar{p}(t)+\tilde{p}(t) \\
q_{m}(t)=\bar{q}_{m}(t)+\tilde{q}_{m}(t) \\
q_{n}(t)=\bar{q}_{n}(t)+\tilde{q}_{n}(t) \\
q_{o}(t)=\bar{q}_{o}(t)+\tilde{q}_{o}(t)
\end{gathered}
$$

where $\bar{p}(t)$ and $\tilde{p}(t)$ correspond respectively to the constant and oscillating components of the active power; $\bar{q}_{m}(t), \bar{q}_{n}(t)$ and $\bar{q}_{o}(t)$ are constant terms associated to the reactive power vector; and $\tilde{q}_{m}(t), \tilde{q}_{n}(t)$ and $\tilde{q}_{o}(t)$ are oscillating components related to the reactive power vector. These power components are referred to as the mno instantaneous power components, with the components related to the active power being calculated as:

$$
\begin{aligned}
& \bar{p}(t)=+v_{m}^{d}(t) i_{m}^{d}(t)+v_{m}^{q}(t) i_{m}^{q}(t)+v_{n}^{d}(t) i_{n}^{d}(t)+v_{n}^{q}(t) i_{n}^{q}(t)+v_{o}^{d}(t) i_{o}^{d}(t)+v_{o}^{q}(t) i_{o}^{q}(t) \\
& \tilde{p}(t)=+v_{m}^{q}(t) i_{m}^{d}(t)+v_{m}^{d}(t) i_{m}^{q}(t)+v_{n}^{q}(t) i_{n}^{d}(t)+v_{n}^{d}(t) i_{n}^{q}(t)+v_{o}^{q}(t) i_{o}^{d}(t)+v_{o}^{d}(t) i_{o}^{q}(t)
\end{aligned}
$$

and the reactive power components given by:

$$
\begin{aligned}
& \bar{q}_{m}(t)=-v_{o}^{d}(t) i_{n}^{d}(t)-v_{o}^{q}(t) i_{n}^{q}(t)+v_{n}^{d}(t) i_{o}^{d}(t)+v_{n}^{q}(t) i_{o}^{q}(t) \\
& \tilde{q}_{m}(t)=-v_{o}^{q}(t) i_{n}^{d}(t)-v_{o}^{d}(t) i_{n}^{q}(t)+v_{n}^{q}(t) i_{o}^{d}(t)+v_{n}^{d}(t) i_{o}^{q}(t) \\
& \bar{q}_{n}(t)=+v_{o}^{d}(t) i_{m}^{d}(t)+v_{o}^{q}(t) i_{m}^{q}(t)-v_{m}^{d}(t) i_{o}^{d}(t)-v_{m}^{q}(t) i_{o}^{q}(t) \\
& \tilde{q}_{n}(t)=+v_{o}^{q}(t) i_{m}^{d}(t)+v_{o}^{d}(t) i_{m}^{q}(t)-v_{m}^{q}(t) i_{o}^{d}(t)-v_{m}^{d}(t) i_{o}^{q}(t)
\end{aligned}
$$




$$
\begin{aligned}
& \bar{q}_{o}(t)=-v_{n}^{q}(t) i_{m}^{d}(t)-v_{n}^{d}(t) i_{m}^{q}(t)+v_{m}^{d}(t) i_{n}^{d}(t)+v_{m}^{q}(t) i_{n}^{q}(t) \\
& \bar{q}_{o}(t)=-v_{n}^{q}(t) i_{m}^{d}(t)-v_{n}^{d}(t) i_{m}^{q}(t)+v_{m}^{d}(t) i_{n}^{d}(t)+v_{m}^{q}(t) i_{n}^{q}(t)
\end{aligned}
$$

In matrix notation, Equations (3.66) to (3.73) can be represented by:

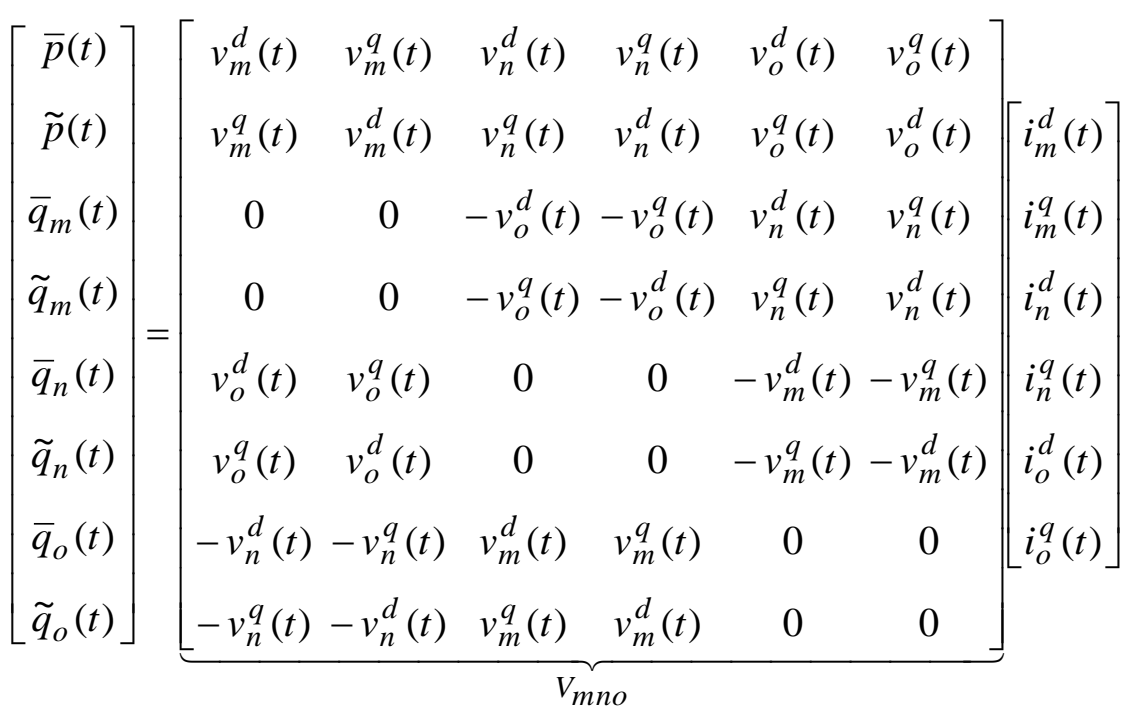

The dot product used to calculate the total instantaneous active power $p(t)$ produces a scalar result, without a geometric interpretation. However, the reactive power vector (3.54) can be expressed as the vector:

$$
\vec{q}(t)=\left[\bar{q}_{m}(t)+\tilde{q}_{m}(t)\right] \hat{m}+\left[\bar{q}_{n}(t)+\tilde{q}_{n}(t)\right] \hat{n}+\left[\bar{q}_{o}(t)+\tilde{q}_{o}(t)\right] \hat{o}
$$

composed by the sum of two other vectors:

$$
\begin{aligned}
& \overrightarrow{\bar{q}}_{m n o}(t)=\bar{q}_{m}(t) \hat{m}+\bar{q}_{n}(t) \hat{n}+\bar{q}_{o}(t) \hat{o} \\
& \overrightarrow{\tilde{q}}_{m n o}(t)=\tilde{q}_{m}(t) \hat{m}+\tilde{q}_{n}(t) \hat{n}+\tilde{q}_{o}(t) \hat{o}
\end{aligned}
$$

which respectively represents the constant and the varying three-phase instantaneous reactive power vector as:

$$
\vec{q}(t)=\overrightarrow{\bar{q}}_{m n o}(t)+\overrightarrow{\tilde{q}}_{m n o}(t)
$$




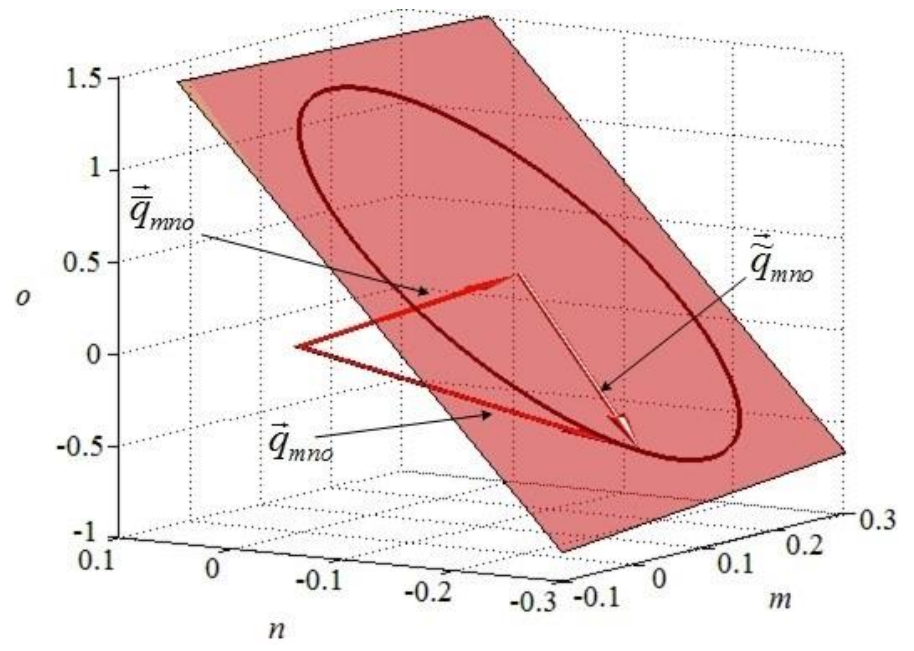

Figure. 3.15: The mno reactive power vector decomposition

Figure 3.15 considers the same steady-state unbalanced case presented before (3.51), illustrating the instantaneous reactive power vector $\vec{q}(t)$ and the locus of the endpoints of $\vec{q}(t)$ in a plane. The reactive power vector $\vec{q}(t)$ is composed of vectors $\overrightarrow{\bar{q}}_{m n o}(t)$, with fixed orientation and its origin at the origin of the mno coordinate system, pointing to the center of the reactive power curve; and $\overrightarrow{\tilde{q}}_{m n o}(t)$, which rotates at twice the system frequency with its origin at the endpoint of $\overrightarrow{\bar{q}}_{m n o}(t)$ and endpoint coincident to the endpoint of $\vec{q}(t)$. For balanced steady-state scenarios, $\left|\overrightarrow{\widetilde{q}}_{m n o}(t)\right|$ is zero, thus $\vec{q}(t)=\overrightarrow{\bar{q}}_{m n o}(t)$.

Figure 3.16 shows the results achieved for the instantaneous mno power components (3.74) related to the unbalanced scenario (3.51). Figure 3.16a shows the active power components (3.62), followed by Figures 3.16b, 3.16c, 3.16d which illustrate the mno reactive power components (3.63), (3.64) and (3.65), respectively. 


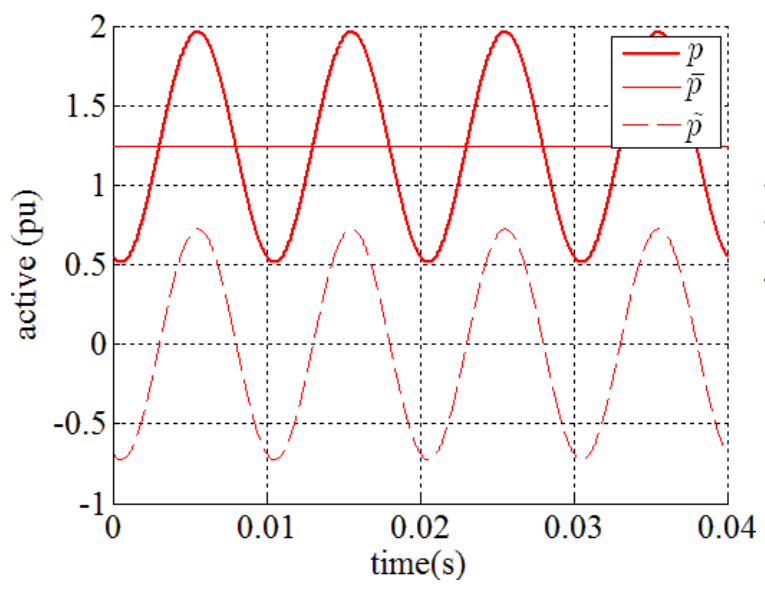

(a)

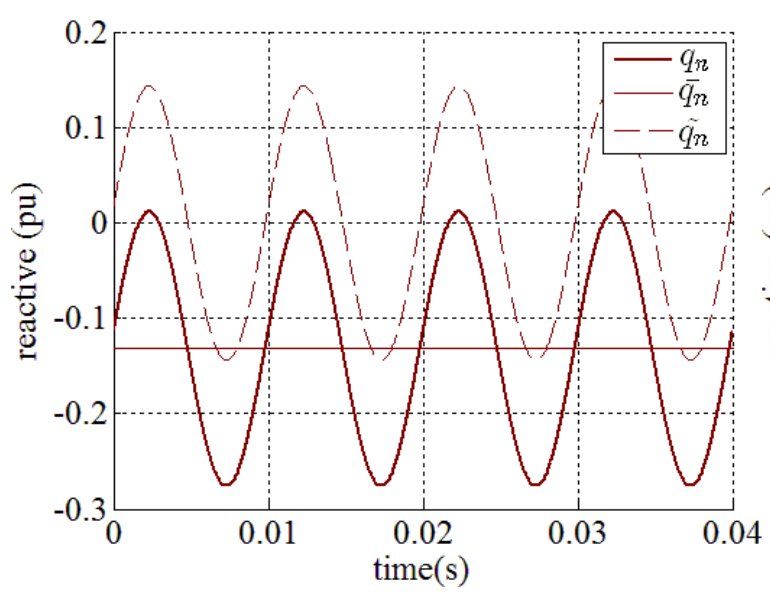

(c)

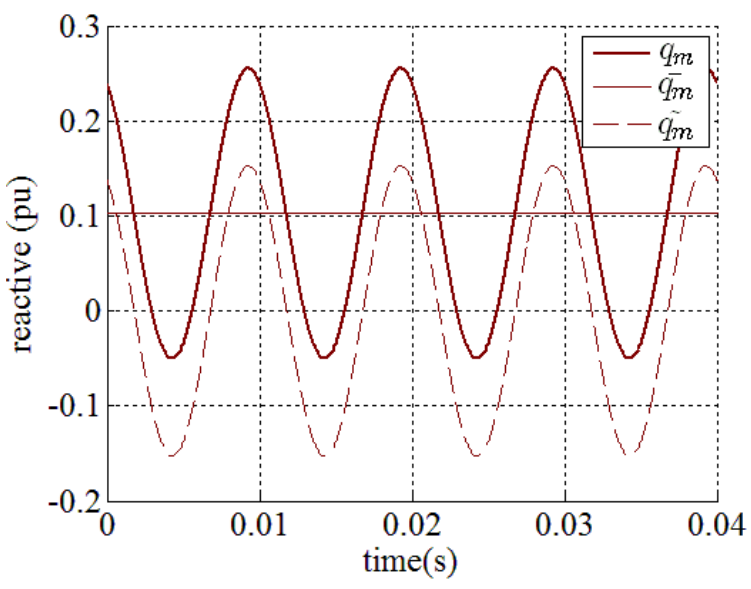

(b)

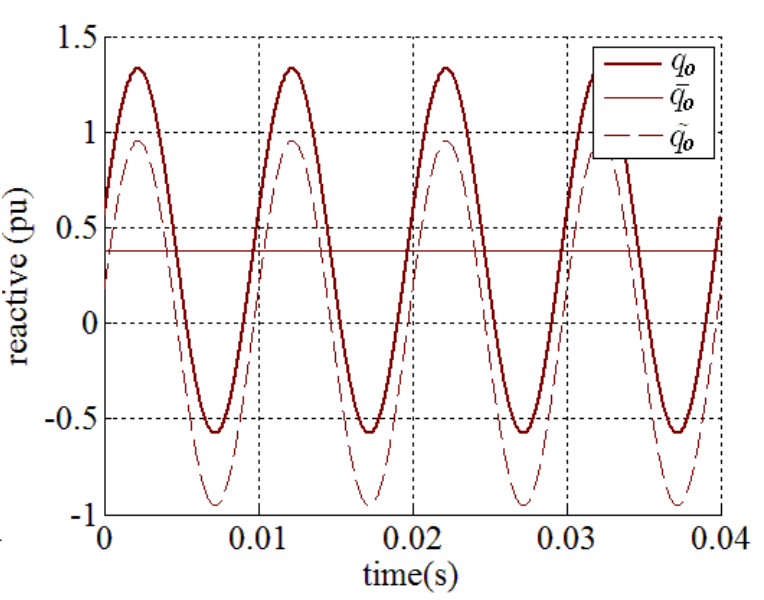

(d)

Figure. 3.16: Instantaneous mno power components: (a) active, (b) $m$ reactive, (c) $n$ reactive, (d) o reactive.

The mno instantaneous power components defined at the above equations provide an unique method to separately calculate the constant and the oscillating terms of the active and reactive powers flowing through the cross section of a general three-phase transmission system.

The next chapter will present applications of these mno power components on voltage sourced converters, proposing algorithms to control the instantaneous power when the converter is connected to an AC network subjected to dynamic conditions. 


\section{Chapter 4}

\section{The mno Instantaneous Power Components Control for Grid}

\section{Connected Voltage Sourced Converters}

Based on the mno theory previously introduced, a novel method capable of controlling the instantaneous power on grid connected voltage sourced converters is presented in this chapter. The advantages of the procedure stand out under unbalanced conditions, mainly when zero sequence voltage and current are present. It enables the system to sustain constant power unlike previous approaches, which either ignore zero sequence power focusing on three-wire systems, or which state that such feature is impossible to be accomplished during unbalanced scenarios with zero sequence power. The technique is applied to general conditions, including balanced or unbalanced scenarios in three-wire systems (without zero sequence power). The performance and effectiveness of the proposed control approach and the proposed power conditioning scheme were demonstrated using electromagnetic transient simulation of a voltage sourced converter connected to an AC system. 


\section{1 - Grid Connected Voltage Sourced Converters}

Voltage sourced converters have been increasingly used to fulfill many needs related to power conditioning in electric networks. These include bulk power transmission over long distances, underwater cable transmission, interconnection between asynchronous subsystems $(50 \mathrm{~Hz}$ and $60 \mathrm{~Hz})$, design of DC grids, integration of distributed generation resources (renewable energy) and energy storage systems, among others [68, 69].

The converter technology is well established in power systems, available not only for HVDC systems (High Voltage Direct Transmission) but also for FACTS applications (Flexible Alternating Current Transmission System), such as STATCOM devices (Static Synchronous Compensator), both at the transmission and distribution level. Such converters enable versatile modes of transmission $[70,71]$. Its application has been

constantly expanded due to the fast development of higher ratings electrical switches, enhanced bridge topologies and advanced robust control strategies [72].

Modern converters make use of the IGBT (Insulated-Gate Bipolar Transistor), a fully controllable electrical switch able to conduct and to interrupt current. This switch is connected with inverse-parallel diodes (free-wheeling) to create reverse conducting switch cells, which provide bidirectional current flow and unidirectional voltage blocking capability. These cells may be connected in series and in parallel in order to meet larger voltage and current requirements, being usually referred to as a valve. The valves are arranged in a structure called bridge, the core interface between the AC and DC subsystems [73].

A typical grid connected voltage sourced converter is essentially composed by the converter bridge, DC capacitors, phase reactors, transformers and filters, as well as the 
control, monitoring, protection and other auxiliary apparatus. Figure 4.1 shows the basic components of such system.

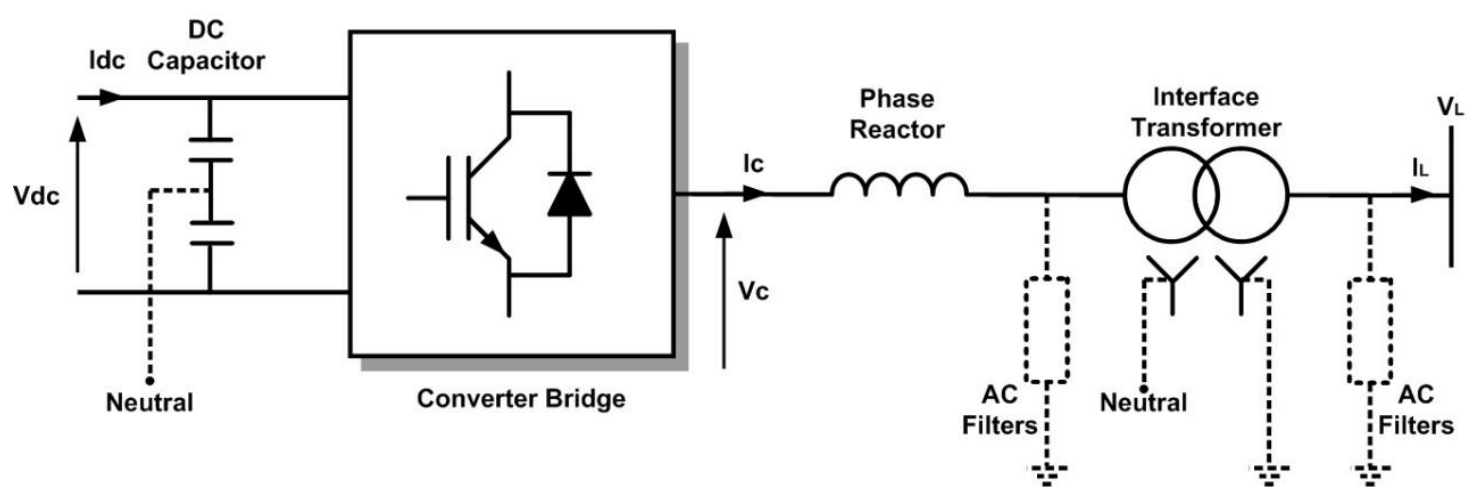

Figure 4.1: Grid connected voltage sourced converter

An advanced feature of voltage sourced converters is the capability of controlling the active and the reactive powers independently. Before discussing the power control technique proposed in this thesis, a brief description is presented in regards to the different possible layouts and connections at the AC/DC interface, as well as their influence on the manner in which the instantaneous power is transferred through the converter, especially during unbalanced voltages and currents.

\subsection{1 - Converter Bridge Topologies and AC/DC Interface Equipment's Connections}

Voltage sourced converters can be designed in a variety of configurations with respect to the converter bridge topology, as well as its connections with the AC and the DC subsystems [73].

The two-level VSC is the simplest bridge model (Figure 4.2a). The voltages at each one of the AC phases are switched between two discrete DC voltage levels. The majority of the VSC systems built in the last decade were based on this arrangement. 
The three-level scheme is the first attempt to improve the deficient harmonic performance of the two-level bridge. It can produce three discrete voltage levels on each phase (Figure 4.2b). They can be designed with capacitor-clamped or diode-clamped, but their practical interest has decreased due to the high complexity when attempting to increase the number of levels beyond three.

(a)

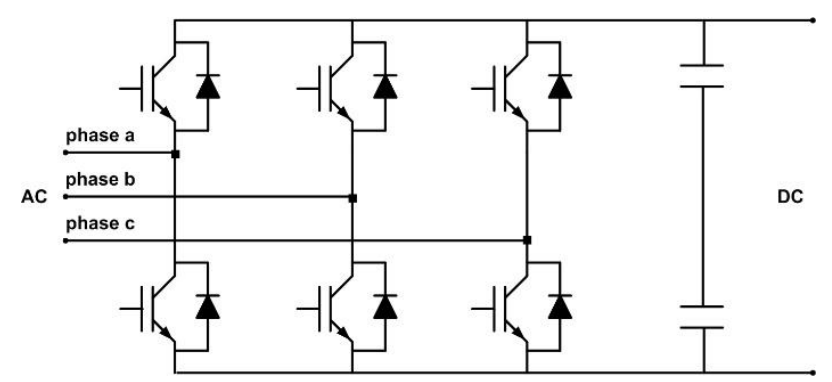

(b)

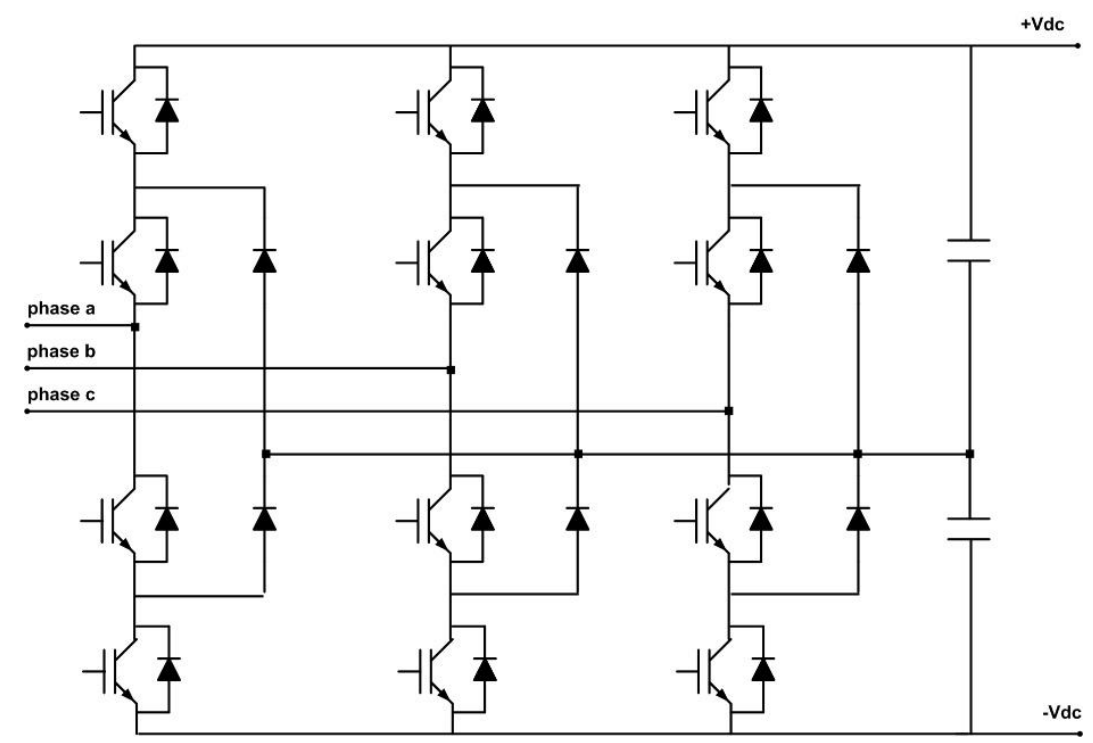

Figure 4.2: VSC bridge topologies: (a) two-level, (b) three-level diode-clamped

The Modular Multilevel Converter (MMC) is the latest and most advanced VSC bridge technology (Figure 4.3). Each valve is composed by independent submodules, commonly designed as half-bridge or full-bridge (also named H-bridge) structures. The converter produces multilevel stepped voltages with low harmonic content at the AC side, 
avoiding the use of AC filters, and even possibly without transformers, offering minimal switching losses.

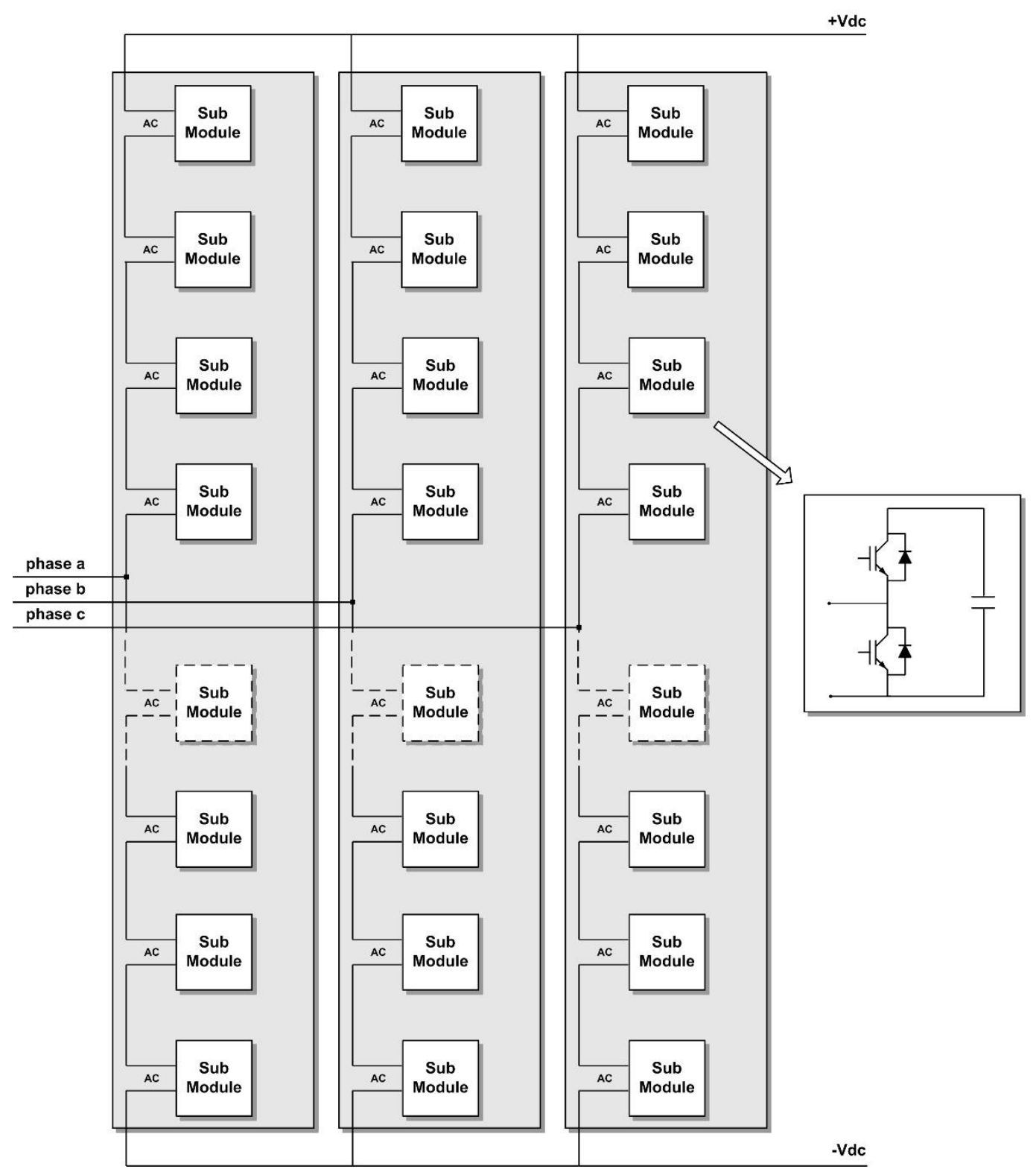

Figure 4.3: Modular Multilevel Converter with half-bridge submodules

On the DC transmission side, the terminals of the bridge may be arranged as monopole, symmetrical monopole, or bipole configuration. Monopole schemes present a single conductor at high voltage, with the other terminal being at ground potential. Hence, the symmetrical monopole is characterized by DC poles connected on each terminal of a 
single converter bridge. The bipole transmission also employs a pair of poles on the DC transmission side, but with two converters bridges at each converter location. Figure 4.4 illustrate these different DC schemes.
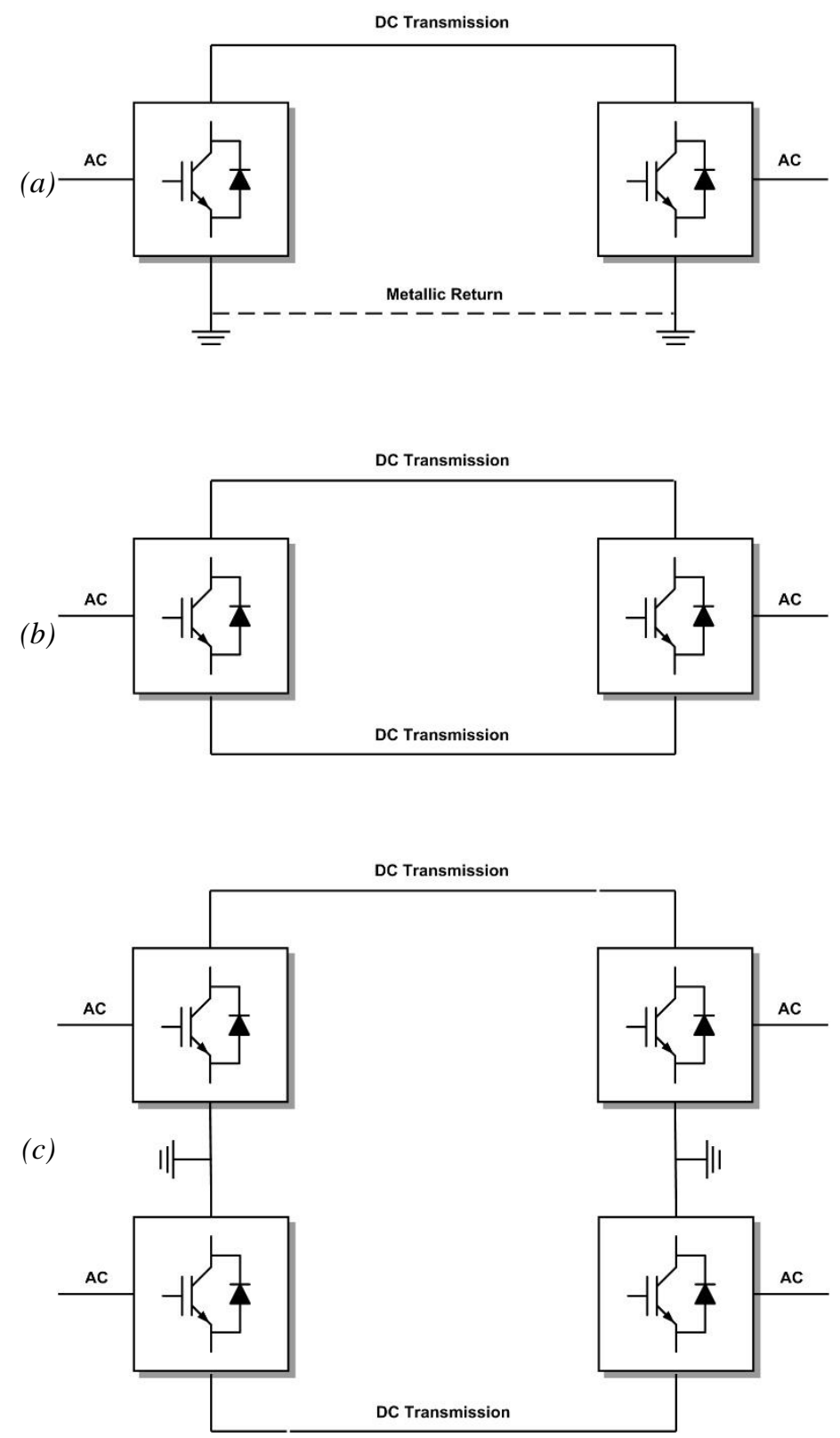

Figure 4.4: Two-terminal VSC configurations: (a) monopole, (b) symmetrical monopole, (c) bipole

Moreover, the DC substations may have different arrangements in regards to the DC network. In a back-to-back system, both converters are at the same location, being commonly employed to allow power transfer between two asynchronous AC systems. The 
two-terminal scheme (point-to-point) is the most common DC configuration used to transfer power over distances. Additionally, voltage sourced converters may be connected into a DC grid as a suitable solution to transmit power among more than two locations using ring, star or meshed DC networks.

The bridge topologies and the connections to the AC and the DC subsystems greatly affect the ability of the transmission system in order to fully control the instantaneous power under unbalanced scenarios. This issue is critical during dynamic transient events involving zero sequence current components, such as remote single-phase faults.

The zero sequence current has not been extensively explored in VSC systems, being typically disregarded by using particular connections that do not allow its flow $[17,74]$. The zero sequence current can be blocked depending on the converter bridge topology and connections between the AC and DC transmission sections. For example, circuits previously shown in Figures 4.2 and 4.3 do not allow zero sequence current to flow.

As it will be shown with the novel control method proposed in this thesis, instead of blocking, if the zero sequence current is permitted to flow during unbalanced transients, then the proposed control makes possible to keep the power flow constant at the precontingency level. It should be noted that for such topologies, the voltage sourced converter must be equipped with robust controls to deal with such zero sequence components, in conformity with standard regulations and overloading limits for the ground and neutral currents.

In converters that permit zero sequence current to flow, besides the traditional connections of each of the three phases of the AC system to the converter bridge, allowing 
positive and negative current components (Figures 4.2 and 4.3), an extra path for the zero sequence current must be provided.

There are different zero sequence current permissive scenarios through the AC/DC interface for the two-level bridge: one strategy is from the midpoint of the capacitors used across the DC side of the VSC, by connecting such point to the neutral or the ground (Figure 4.5a); another design relates to the four-leg converter, which has an additional leg connected to the neutral (Figure 4.5b).

(a)
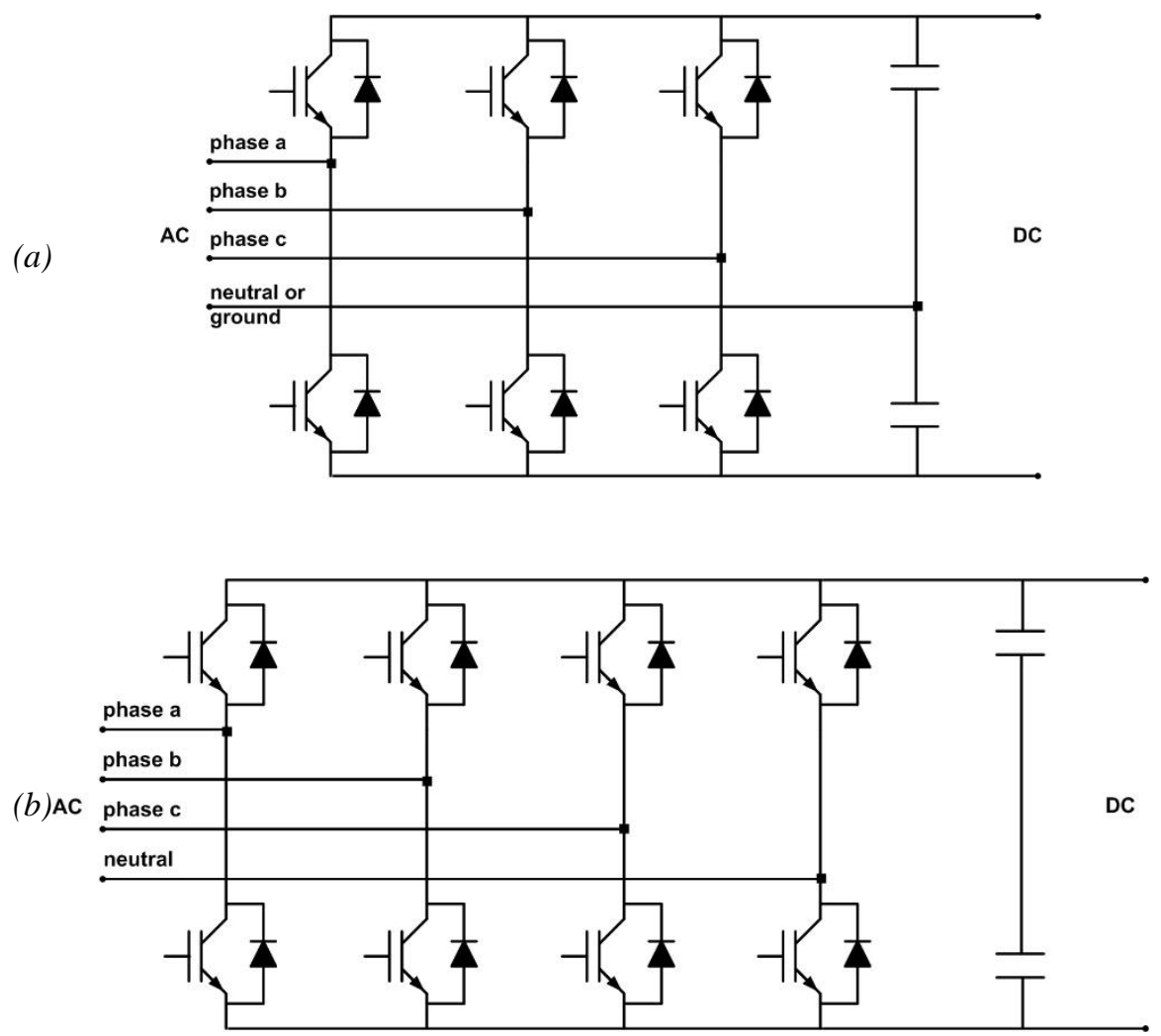

Figure 4.5: Two-level voltage sourced converter zero sequence current permissive connections:

(a) midpoint of DC capacitors, (b) four-leg converter

The four-leg topology can also be extended to the half-bridge MMC, adding an extra leg to the structure shown in Figure 4.3. Another configuration for the multilevel converter refers to the cascade H-bridge wye connected. In this case, the submodules must be designed with H-bridge structures because of its capability to output bipolar voltages, 
considering the AC phases are connected to one terminal of the converter's legs in a wye configuration (Figure 4.6).

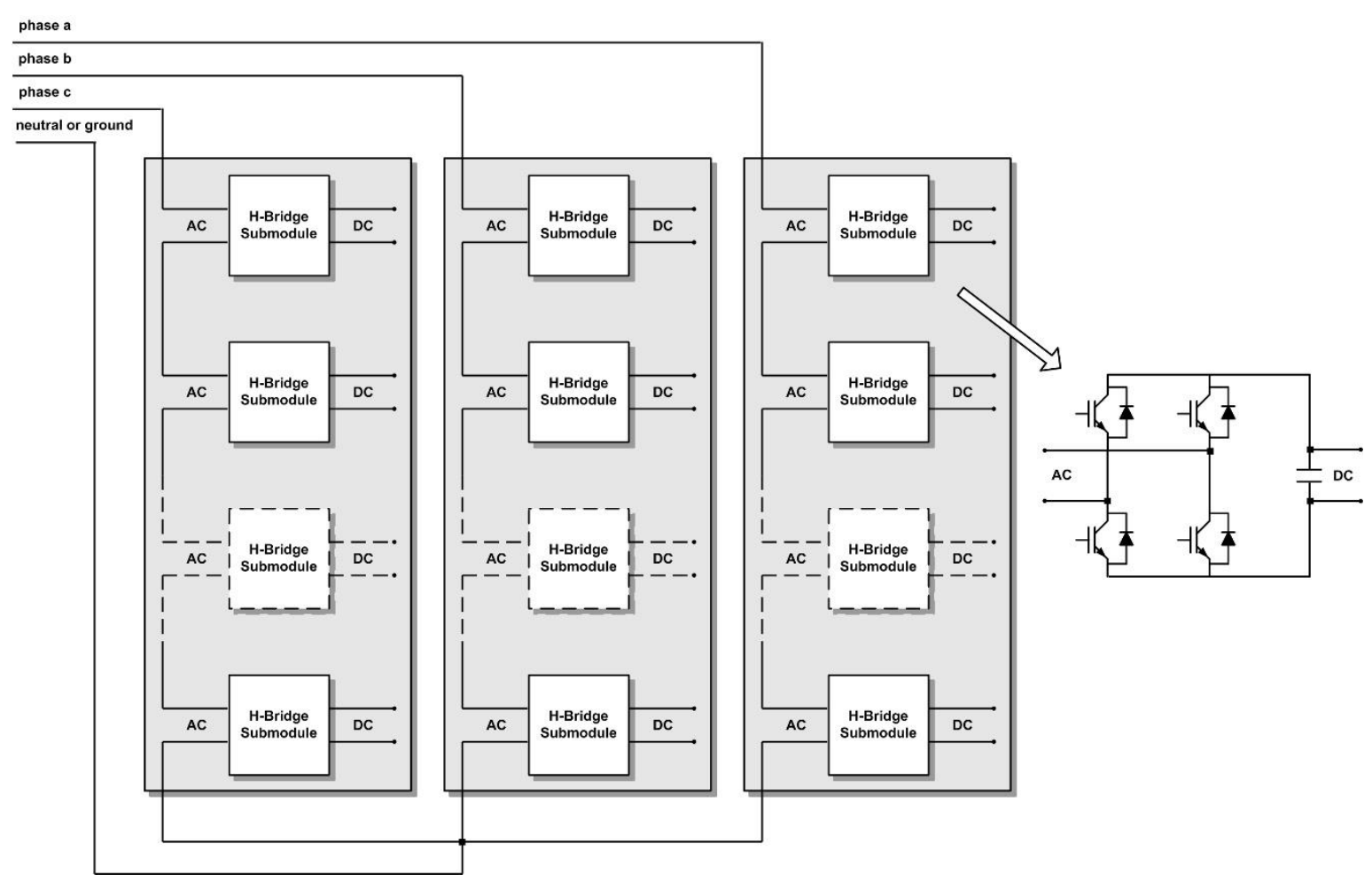

Figure 4.6: MMC VSC with cascade H-bridge wye connected

Furthermore, the substation apparatus connected in between the PCC (Point of Common Coupling) and the AC terminals of the bridge, such as power transformers, phase reactors and filters, must also be considered. The transformer plays an essential role, although new MMC topologies present transformerless designs. However, if a transformer is required, it must be zero sequence current permissive.

It is common to block zero sequence currents, as stated previously, with the use of delta transformer connections, focusing the analyses on three-wire systems. However, this type of winding connection may lead to overvoltage, for example during transient singlephase faults [74]. Delta configurations may also lead to DC voltage imbalance (demanding use of choppers, for instance), as a consequence of pole-to-ground faults [74]. For all these 
cases, the high impedance to the zero sequence makes fault detection more difficult due to the small value of the fault current.

One alternative arrangement for the zero current path through the transformer is the wye-wye configuration, which allows connections with the neutral point of the wye winding topology. Another possible configuration is the use of zig-zag transformers, also called T-connected grounding transformers, usually employed by the addition of a wyedelta transformer with grounded neutral and the delta windings terminals in open circuit, providing low impedance path for the zero sequence current [75].

Once the equipment's layouts that allow all the current sequences to flow through the voltage sourced converter are described, it is time to move forward towards the control over these current components. The following sections present a background of voltage sourced converter control techniques.

\subsection{2 - Classical Control Techniques}

The control structures developed for voltage sourced converters are directly or indirectly related to the control of the AC current and the DC voltage. The VSC works with a constant voltage polarity on its DC side (reason for the converter's own name) but allows bidirectional flow of DC current, and therefore the electric power can also flow bidirectionally. On the AC side, the current through the reactance (phase reactors and transformer) is established by the voltage difference between the AC system voltage $v_{S}(t)$ and the controlled AC converter voltage $v_{c}(t)$ (Figure 4.7). The model for the interconnection with the AC side is given by: 


$$
v_{c}(t)=v_{s}(t)+R i(t)+L \frac{d i(t)}{d t}
$$

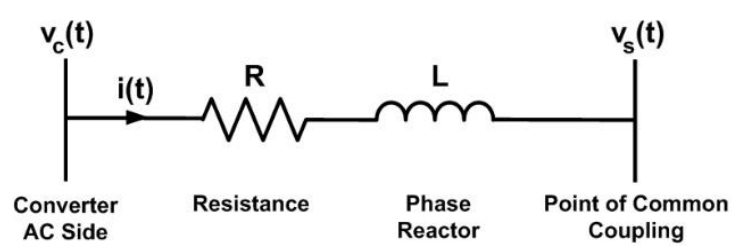

Figure 4.7: Converter connection model of the VSC to the AC network

The magnitude and phase angle of the converter AC side voltage can be independently adjusted by switching the converter valves using PWM techniques (Pulse Width Modulation). The modulating signals are replicas of the phase voltages that are to be applied, being synthesized by the main control system of the converter, which is monitoring electric variables at the AC/DC interface. The active and reactive powers can be controlled by regulating the converter voltage magnitude and phase angle as:

$$
\begin{gathered}
P=\frac{V_{s} V_{c} \sin \phi}{X} \\
Q=\frac{V_{s}^{2}}{X}-\frac{V_{s} V_{c} \cos \phi}{X}
\end{gathered}
$$

where $V_{s}$ and $V_{c}$ are respectively the voltage magnitude of the system and the converter, $X$ is the equivalent reactance, and $\phi$ is the phase angle difference between $v_{s}(t)$ and $v_{c}(t)$. Hence, the modulating signal has equal magnitude $V_{c}$ for the three phases, and equal phase displacement $\phi$ in regards to respective phases of the system voltages.

The first control methods developed for VSC focused just on balanced AC scenarios, aiming to establish a balanced set of voltages (positive sequence) at the point of common coupling (PCC). This strategy allows only two degrees of freedom, i.e. the magnitude and the phase shift of the converter voltage with reference to the system voltage. 
The well-known direct control and vector control are the main techniques proposed to accomplish this task [73].

The direct method controls two variables of the system, which for example can be the active power and the reactive power, based on Equations (4.2) and (4.3). This is achieved by proper selection of the magnitude $V_{c}$ and the phase shift $\phi$. The AC voltage can also be controlled through the reactive power, and the DC side voltage can be controlled through the active power, depending on the VSC application. The two measured values are compared to the two respective reference orders, with the two output errors being processed by separated controllers, usually PI controllers (Proportional Integral Controllers). These controllers synthesize the signals for the modulation section, typically a PWM (Pulse Width Modulation) which thereafter generates the pulses for the switching components [76], in order to change the magnitude and the phase angle of the three-phase voltage at the converter terminals. This method has inherent cross-coupling between the active and reactive current components, and hence a change in the active power order causes a transient in the reactive power and vice-versa. Unfortunately, it also does not have the capability to limit the current magnitude.

Alternatively, the vector control approach [76] converts the $a b c$ three-phase voltages and currents into a $d q$ reference frame by using the reduced Park's transformation (Section 2.2.4). It presents the advantage of controlling two chosen variables independently. The cross-coupling is removed by feed-forward control loops. Two $d q$ current references are generated from two separate outer PI controllers which are processing the errors from two measured variables, compared with their respective reference setpoints. These $d q$ current references are compared to the respective measured 
system currents in the $d q$ domain, with the errors adjusted by other two fast inner PI controllers. Such conventional vector control utilizes four PI controllers (two outer and two inner controllers). The $d q$ equations for the same model shown in Figure 4.7 are given by:

$$
\left\{\begin{array}{l}
\frac{d i_{d}(t)}{d t}=\frac{1}{L}\left[-R i_{d}(t)-v_{s d}(t)+v_{c d}(t)\right]-\omega i_{q}(t) \\
\frac{d i_{q}(t)}{d t}=\frac{1}{L}\left[-R i_{q}(t)-v_{s q}(t)+v_{c q}(t)\right]+\omega i_{s}(t)
\end{array}\right.
$$

Unfortunately, a major drawback of these both classical control techniques is the high sensitivity to grid disturbances and, consequently, the deficient performance under unbalanced AC scenarios. The control for unbalanced conditions is a challenging issue, demanding enhanced schemes to better accommodate dynamic transient events.

\subsection{3 - Control Methods under Adverse AC Conditions}

The three-phase voltage and three-phase current at the AC side of the converters are far from being continuously and perfectly balanced during the power delivery. Many dynamic events and different load conditions force the system to unbalanced scenarios, which generate undesirable oscillations in the power transfer.

The voltage sourced converters should be able to withstand impacts in the power flow, riding through disturbances in accordance with limits set by applicable grid codes and avoiding unnecessary disconnections by the protection schemes. Additionally, the VSC is also expected to deal with harmonic distortion, following network recommendations and standards [13-20].

The classical controllers are not able to handle general AC grid conditions, as previously mentioned. Therefore, several solutions have been explored in the past, by 
appropriately injecting synthesized currents to attenuate power oscillations. These control strategies include rotating reference frames $[86,87]$ with proportional-integral controllers $[85,88]$, stationary frames [17] with proportional-resonant controllers [16, 89], as well as nonlinear hysteresis and predictive deadbeat controllers, among others [90].

Significant studies regarding the control under unbalanced and distorted AC conditions date from the 1990 s, with the first analyses focusing on tracking the positive and negative reference currents with one single controller [81, 82]. Developments were made based on two independent control loops for each sequence (positive and negative) through double synchronous reference frame current controllers [83, 84]. This permitted a reduction in the oscillations in the transmitted power when using $d q$ signals generated with Park's transformation $[85,91,92]$.

The current controllers based on individual reference frames for positive and negative sequence have a good performance (four independent degrees of freedom), especially the ones based on the instantaneous power theory $[18,19]$. Nevertheless, dealing with zero sequence transients is still complicated due to the intrinsic oscillating behavior of the zero sequence power [65], which affects the total three-phase energy flow per time unit. This is one of the reasons why zero sequence content is not welcome in most circuits, being frequently avoided [65]. The $p-q$ theory states that it is not possible to independently control the constant and oscillating power terms (Chapter 2). Indeed, in many circumstances, certain circuit connections are conveniently chosen to prevent the zero sequence current circulation.

Fortunately, the mno power theory introduced in Chapter 3 allows the zero sequence current to be controlled in order to guarantee a constant power transfer. 
Subsequently, a method able to independently control the constant and oscillating terms of the instantaneous power in voltage sourced converters systems is presented. The mno instantaneous power components control allows the converter to independently control the current magnitude and phase angle of each of the three $a b c$ phases individually. Simulation results are presented in order to verify the proposed control structure.

\section{2 - The mno Instantaneous Power Components}

\section{Control}

The mno instantaneous power components control can be used to generate the required instantaneous current on each phase of the AC side of the voltage sourced converter to match a specific operating requirement, such as sustaining a constant power flow, minimizing the propagation of power disturbances and oscillations during unbalanced conditions. The advantages of the procedure stand out under unbalanced scenarios characterized by concomitant presence of zero sequence voltage and zero sequence current content.

The $d q$ mno instantaneous current components reference vector $\vec{i}_{m n o}^{d q^{*}}(t)$ is synthesized at the mno coordinates domain from the constant power orders given through the mno instantaneous power components vector $\vec{p}^{*}(t)$ and also based on the $d q$ mno instantaneous voltage components vector $\vec{v}_{m n o}^{d q}(t)$ evaluated from the measured voltage $\vec{v}_{a b c}(t)$ at the point of common coupling. The necessary phase current vector $\vec{i}_{a b c}^{*}(t)$ at the 
original $a b c$ coordinate system are then generated by applying the inverse mno transformation.

The phase currents can be easily controlled by using a hysteresis current controller [77]. Due to its simplicity, the hysteresis current controller was selected to be applied, although other control techniques can be employed to implement the current controller, such as proportional resonant controllers [78]. Details about the hysteresis current controller implementation will be provided at Section 4.2.5. Figure 4.8 illustrates the mno instantaneous current control scheme for a voltage sourced converter connected to the AC network.

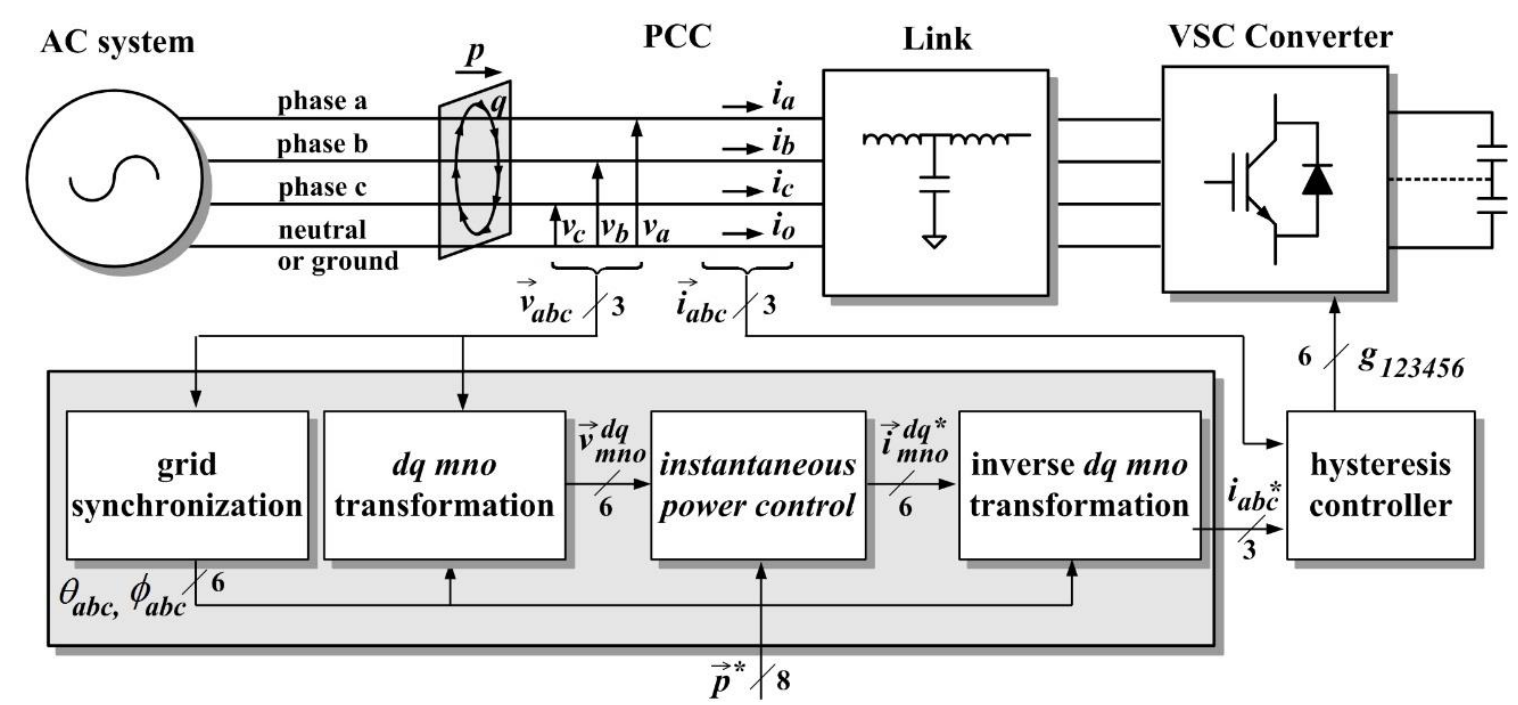

Figure 4.8: The mno instantaneous power components control scheme with hysteresis current controller

Although a two-level converter topology is assumed, the method is conceptually applicable to different converter topologies (e.g., multi-level converters), as the interactions with the $\mathrm{AC}$ grid remains equivalent concerning the connections which allow the positive, negative and zero sequence current components to flow. 
In sequence, each section of the proposed control method is introduced, along with simulation results to illustrate the mno power components control. Before describing control details, the characteristics of the test system are introduced.

\subsection{1 - The Grid Connected Voltage Sourced Converter Test System}

The operational principle of the control proposed in this work is tested in a grid connected voltage sourced converter as shown in Figure 4.1, with the control concept as shown in Figure 4.8. The two-level three-phase converter bridge topology is designed as illustrated in Figure 4.5a, connected to the AC network using a wye-wye transformer.

The converter has a nominal power rating of $400 \mathrm{MW}$ with nominal DC voltage at $\pm 320 \mathrm{kV}$. The converter is operated to generate the specified active power and reactive power infeed to the AC network. The converter is part of a DC transmission system where the remote converter regulates the DC voltage to its rated value.

The DC capacitors time constant is set to $2 \mathrm{~kJ} / \mathrm{MVA}$, calculated by dividing the energy stored in the capacitor at rated DC voltage by the rated power of the converter. The converter transformer has a nominal turn's ratio of $1: 1$ with 0.1 pu leakage impedance. The neutral of the transformer is connected to the capacitors midpoint on the DC side, which alternatively can also be grounded.

Phase reactors were designed with an impedance of $0.12 \mathrm{pu}$ to interface the converter with the AC network, providing adequate fault current limitation from $\mathrm{AC}$ side short circuits and also attenuation to the high frequency current ripple. As a tradeoff, a large reactor provides lower current ripple, however it slows down the dynamics of the converter. 
On the other hand, a small reactor increases the dynamics response, allowing higher current ripple.

High pass-filters are connected as shunt elements between the converter and the transformer, reducing harmonic content from the bridge switching devices and protecting the power transformer.

The AC network connected at the point of common coupling is represented by Thevenin's equivalent of a $345 \mathrm{kV}$ voltage source at $50 \mathrm{~Hz}$ with a series impedance to express value five (5) of short circuit ratio.

\subsection{2 - Grid Synchronization}

Successful performance of the proposed control structure depends on the synchronization system, responsible to generate reference signals that constantly and rapidly update the controller. The mno theory considers the voltage as a time dependent vector at the mno three-dimensional cartesian reference frame. Therefore, synchronization is established by tracking the mno angles of the grid voltage $\theta_{a}(t), \theta_{b}(t), \theta_{c}(t), \phi_{a}(t)$, $\phi_{b}(t)$ and $\phi_{c}(t)$, which enables the instantaneous update of the mno reference frame, and, therefore, the calculation of the mno components, as explained in the Chapter 3.

The synchronization scheme can be subdivided in three steps, following the procedures described in Section 3.2 of Chapter 3: calculation of the instantaneous voltage tangent vector (normalized derivative), generation of the instantaneous normal vector to yield the basis vector $\hat{o}$, and calculation of the mno angles. Figure 4.9 illustrates the synchronization section. 


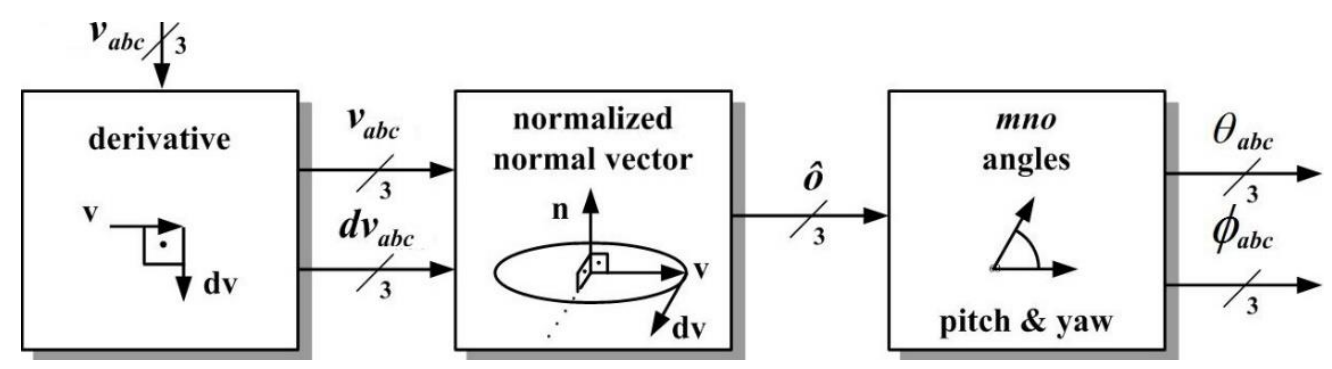

Figure 4.9: The mno grid synchronization

The derivative vector components are obtained by generating signals in quadrature with the phase voltages. Different techniques for the QSG (Quadrature Signal Generator) can be employed. The method adopted in this thesis is based on a SOGI structure (Second Order Generalized Integrator) which is able to process the signals similar to a delay-free filter [93]. Figure 4.10 gives a block diagram of the SOGI scheme applied to each measured phase voltage of the system.

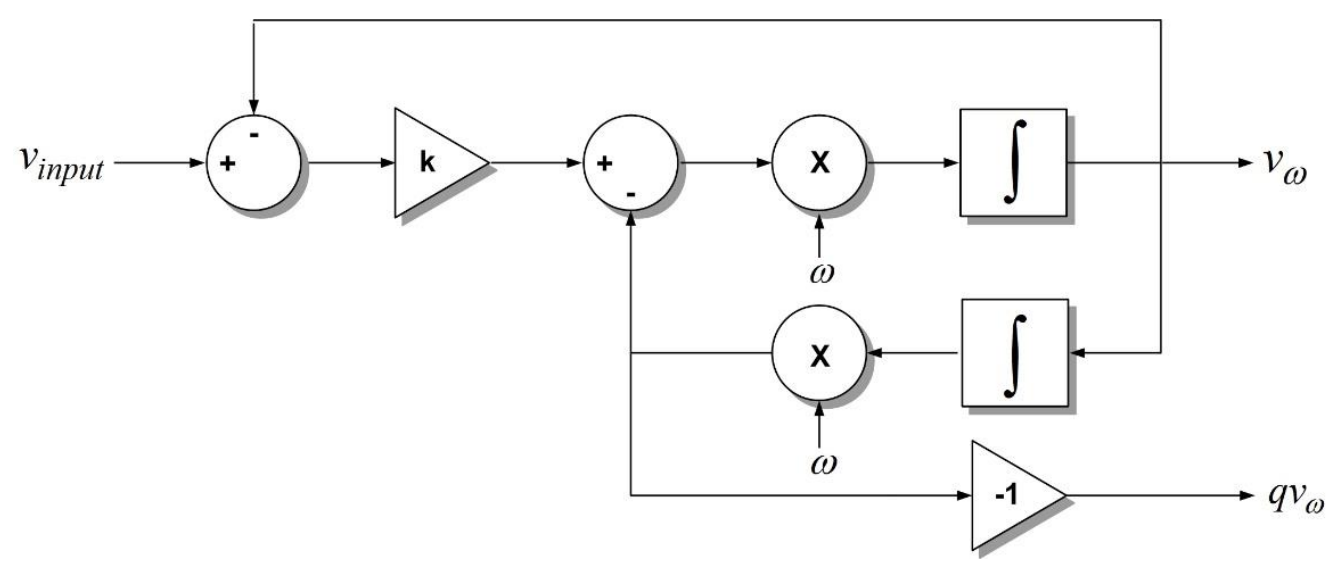

Figure 4.10: SOGI-QSG control diagram

The transfer functions for the components in phase and in quadrature are:

$$
\begin{aligned}
& \frac{v_{\omega}(s)}{v_{\text {inрut }}(s)}=\frac{k \omega s}{s^{2}+k \omega s+\omega^{2}} \\
& \frac{q v_{\omega}(s)}{v_{\text {input }}(s)}=\frac{k \omega^{2}}{s^{2}+k \omega s+\omega^{2}}
\end{aligned}
$$


where $v_{\text {input }}(s)$ corresponds to one of the phase voltages of the $a b c$ three-phase voltage; $v_{\omega}(s)$ and $q v_{\omega}(s)$ corresponds, respectively, to the component in phase and in quadrature (derivative) of the input phase voltage at the frequency $\omega ; q$ refers to the quadrature operator; and $k$ is an appropriately selected filter gain designed to provide unitary quality factor. The Equations (4.5) and (4.6) are initially tuned at the nominal frequency, where the gains are unitary:

$$
\left.\frac{v_{\omega}(s)}{v_{\text {input }}(s)}\right|_{s=\omega j}=\left.1 \quad \frac{q v_{\omega}(s)}{v_{\text {input }}(s)}\right|_{s=\omega j}=-j
$$

Figure 4.11 shows the effects of noise on the input voltage signal $v_{\text {input }}$ of the SOGIQSG structure of Figure 4.10. White noise with uniform frequency distribution and peak amplitude equal to $20 \%$ of an undistorted voltage at fundamental frequency is added on the $v_{\text {input }}$ at $550 \mathrm{~ms}$.
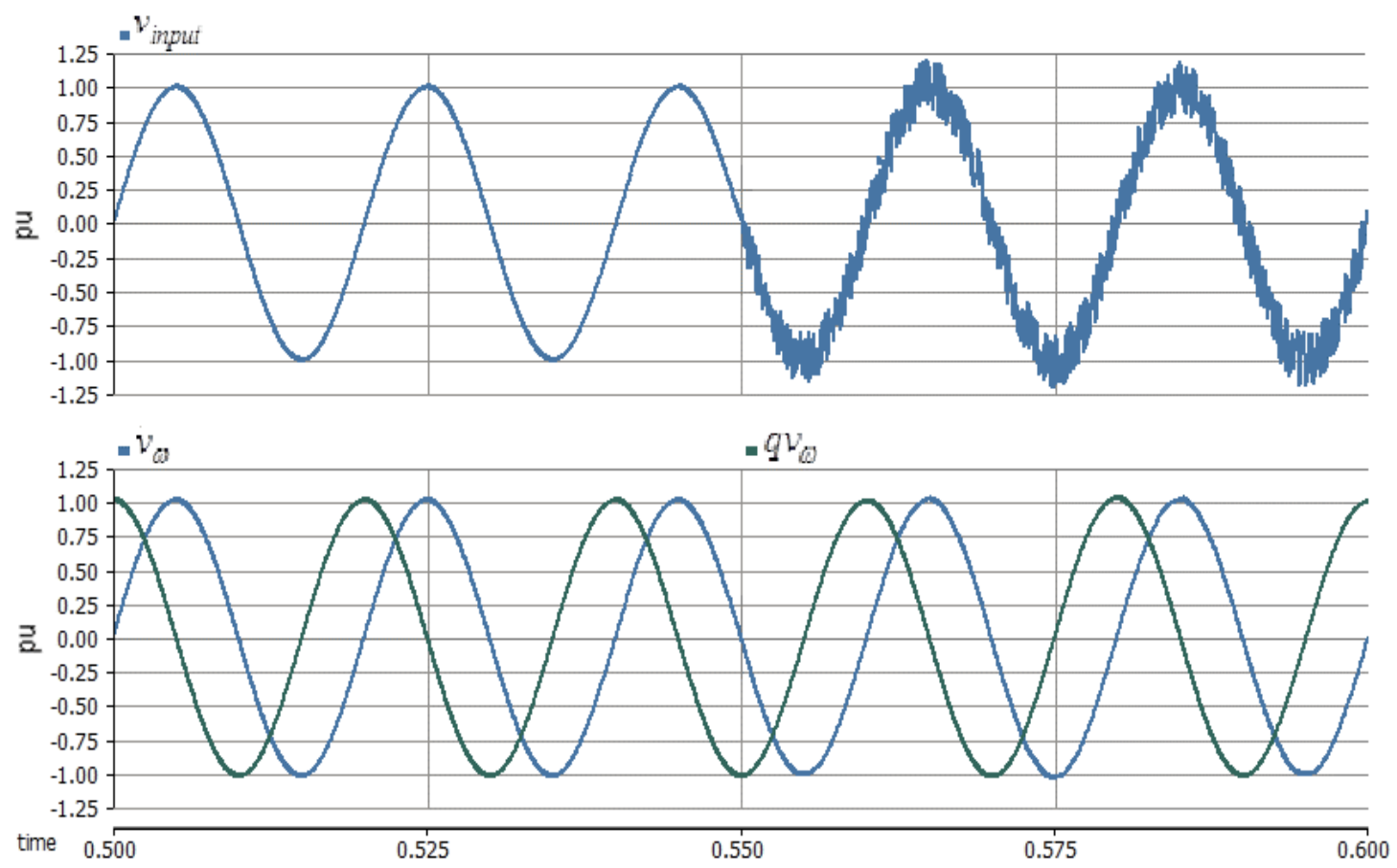

Figure 4.11: SOGI-QSG response for noise effects 
The direct $v_{\omega}$ and quadrature voltages $q v_{\omega}$ remain essentially unperturbed with such additive noise, thereby demonstrating the strong noise rejection capability of the SOGI-QSG. The reason for this is that measurement noise typically has high frequency components and the SOGI-QSG is highly discriminatory to frequency components higher than the tuned frequency. Harmonic distortion can be analyzed using a M-SOGI-QSG (Multiple Second Order Generalized Integrator Quadrature Signal Generator), a control structure able to synchronize multiples frequencies of the system voltage [94].

Figure 4.12 illustrates the SOGI-QSG performance for a 0.8 pu voltage dip along with a phase shift of $\pi / 6$ at phase $a$ between $450 \mathrm{~ms}$ and $650 \mathrm{~ms}$, with the input voltage $v_{\text {input }}$ of the control structure of Figure 4.10 equals to the voltage at phase $a v_{a}(t)$.

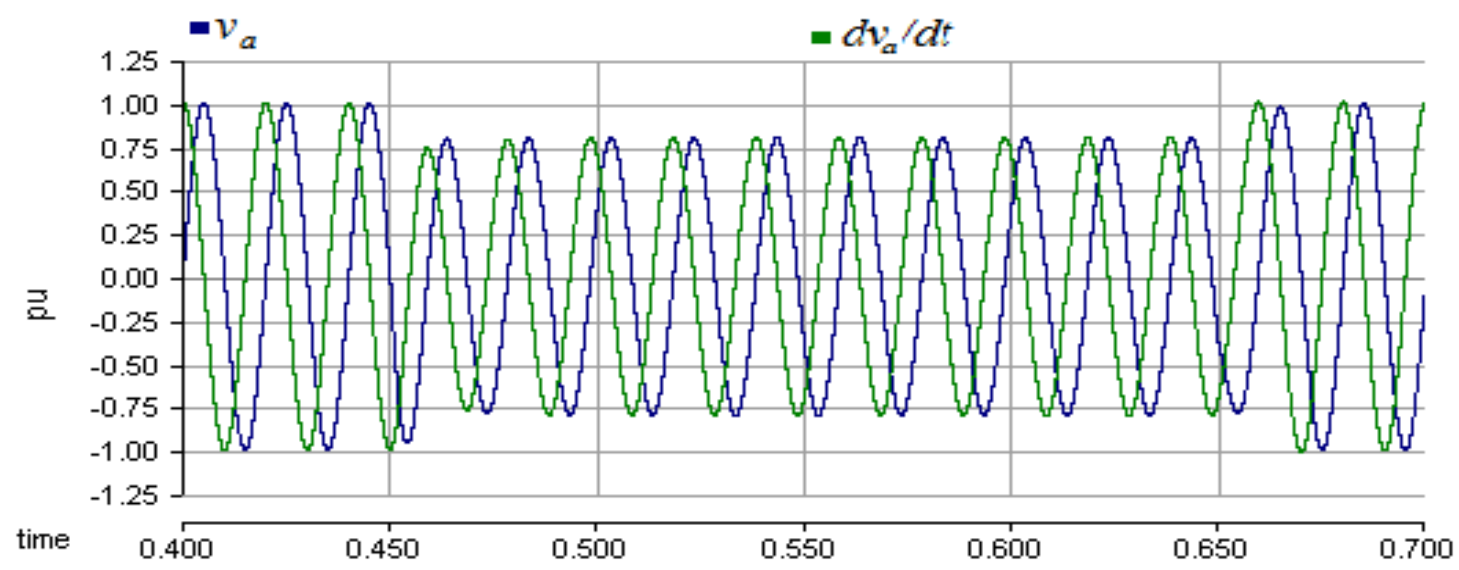

Figure 4.12: SOGI-QSG response for voltage sag and phase shift

This method is applied to each phase voltage $v_{a}(t), v_{b}(t)$ and $v_{c}(t)$, generating derivative of these input voltages $\frac{d v_{a}(t)}{d t}, \frac{d v_{b}(t)}{d t}$ and $\frac{d v_{c}(t)}{d t}$, which are the components of the instantaneous voltage tangent vector $\frac{d \vec{v}_{a b c}(t)}{d t}$. 
The next step generates the normal vector by calculating the vector product between the voltage vector $\vec{v}_{a b c}(t)$ and the derivative voltage vector $\frac{d \vec{v}_{a b c}(t)}{d t}$ according to Equation (3.17) of Section 3.2.2. The components of the normal vector are calculated by the equations:

$$
\begin{aligned}
& n_{a}(t)=v_{b}(t) \frac{d v_{c}(t)}{d t}-\frac{d v_{b}(t)}{d t} v_{c}(t) \\
& n_{b}(t)=v_{c}(t) \frac{d v_{a}(t)}{d t}-\frac{d v_{c}(t)}{d t} v_{a}(t) \\
& n_{c}(t)=v_{a}(t) \frac{d v_{b}(t)}{d t}-\frac{d v_{a}(t)}{d t} v_{b}(t)
\end{aligned}
$$

The normal vector components are normalized to yield:

$$
\begin{aligned}
& o_{a}(t)=\frac{n_{a}(t)}{\sqrt{\left(n_{a}(t)\right)^{2}+\left(n_{b}(t)\right)^{2}+\left(n_{c}(t)\right)^{2}}} \\
& o_{b}(t)=\frac{n_{b}(t)}{\sqrt{\left(n_{a}(t)\right)^{2}+\left(n_{b}(t)\right)^{2}+\left(n_{c}(t)\right)^{2}}} \\
& o_{c}(t)=\frac{n_{c}(t)}{\sqrt{\left(n_{a}(t)\right)^{2}+\left(n_{b}(t)\right)^{2}+\left(n_{c}(t)\right)^{2}}}
\end{aligned}
$$

which corresponds to the components of the unit basis vector $\hat{o}(t)$ :

$$
\hat{o}(t)=o_{a}(t) \hat{a}+o_{b}(t) \hat{b}+o_{c}(t) \hat{c}
$$

The performance of the synchronization structure is illustrated by using values of the same illustrative example presented in Chapter 3, with positive, negative and zero sequence content (Section 3.2.7). Therefore, the unbalanced voltage scenario: 


$$
\left\{\begin{array}{l}
v_{a}(t)=0.8 \cos (\omega t-\pi / 6) \\
v_{b}(t)=\cos (\omega t-2 \pi / 3) \\
v_{c}(t)=\cos (\omega t+2 \pi / 3)
\end{array}\right.
$$

is simulated for 10 cycles, from $450 \mathrm{~ms}$ to $650 \mathrm{~ms}$. Figure 4.13 shows the three-phase voltage $\vec{v}_{a b c}(t)$ along with the correspondent basis vector components $o_{a}(t), o_{b}(t)$ and $o_{c}(t)$ obtained by control using the control structures early described.

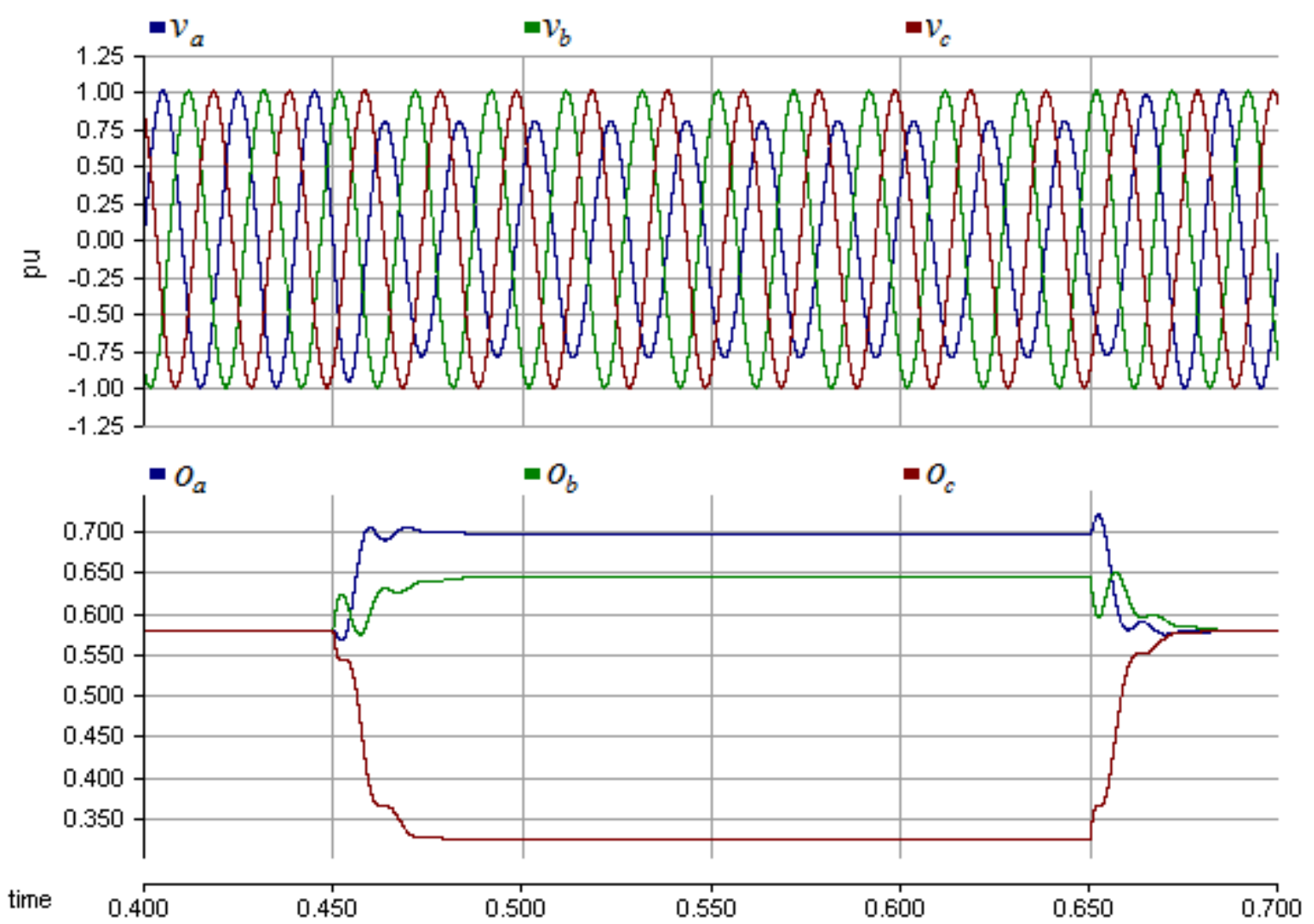

Figure 4.13: Unbalanced voltage for Equation (4.15) and correspondent basis vector components $\hat{o}(t)$

Once the normal vector, and consequently the unit basis vector $\hat{o}(t)$, is accurately tracking the voltage dynamics of the system, the mno angles can be updated. This calculation is performed using the equations presented at Section 3.2.3 (Chapter 3). The angle $\phi_{a}(t)$ is set to zero, attaching the phase angle references between the voltages $v_{a}(t)$ 
and $v_{m}(t)$, as explained in Chapter 3. Figure 4.14 shows the mno pitch angles $\theta_{a}(t), \theta_{b}(t)$ and $\theta_{c}(t)$, as well as the mno yaw angles $\phi_{a}(t), \phi_{b}(t)$ and $\phi_{c}(t)$ for the unbalanced voltage case of Equation (4.15).

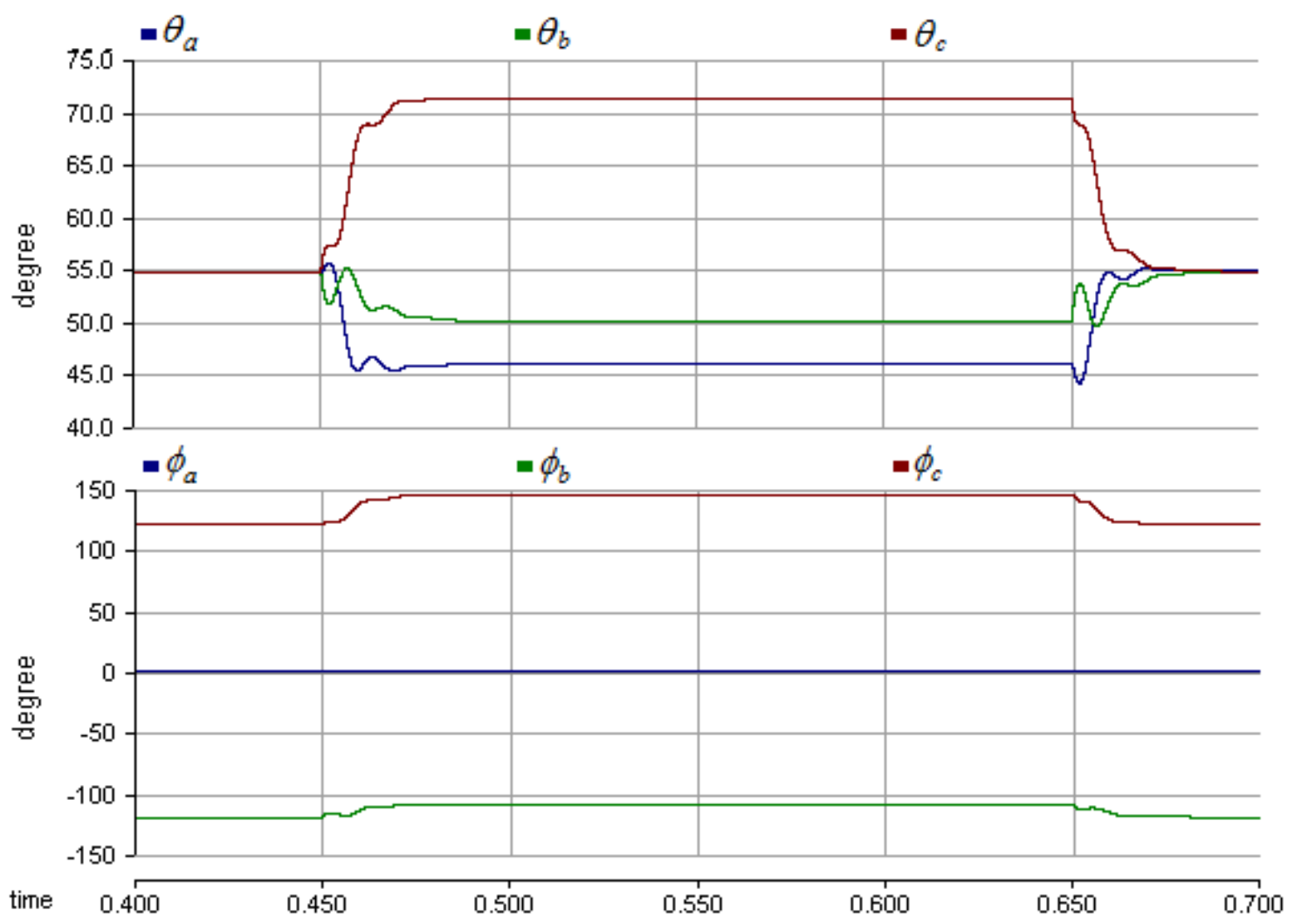

Figure 4.14: The mno angles components for Equation (4.15): mno pitch angles and mno yaw angles

The unbalanced disturbance can be rapidly detected within $15 \mathrm{~ms}$ (approximately at $465 \mathrm{~ms}$ ), with the control structure accurately synchronized to the dynamic conditions. On the removal of the unbalanced condition at $650 \mathrm{~ms}$, the synchronization signals return to their balanced values approximately within $15 \mathrm{~ms}$.

Table 4.1 shows numerical values of the components of the unit basis vector $\hat{o}(t)$ and the mno angles for both conditions (balanced and unbalanced) once the transients between the conditions are not present. 


\begin{tabular}{|c|c|c|c|c|c|c|c|c|c|}
\hline & \multicolumn{3}{|c|}{ Unitary basis vector $\hat{o}$} & \multicolumn{3}{c|}{ mno Pitch Angles } & \multicolumn{3}{c|}{ mno Yaw Angles } \\
\hline $\begin{array}{c}\text { AC voltage } \\
\text { condition }\end{array}$ & $o_{a}(t)$ & $o_{b}(t)$ & $o_{c}(t)$ & $\theta_{a}(t)$ & $\theta_{b}(t)$ & $\theta_{c}(t)$ & $\phi_{a}(t)$ & $\phi_{b}(t)$ & $\phi_{c}(t)$ \\
\hline $\begin{array}{c}\text { Balanced } \\
(\mathrm{t}=400 \mathrm{~ms})\end{array}$ & 0.5773 & 0.5773 & 0.5773 & $54.73^{\circ}$ & $54.73^{\circ}$ & $54.73^{\circ}$ & $0^{\circ}$ & $120.00^{\circ}$ & $-120.00^{\circ}$ \\
\hline $\begin{array}{c}\text { Unbalanced } \\
(\mathrm{t}=500 \mathrm{~ms})\end{array}$ & 0.6956 & 0.3213 & 0.6426 & $45.92^{\circ}$ & $71.26^{\circ}$ & $50.02^{\circ}$ & $0^{\circ}$ & $109.18^{\circ}$ & $-144.29^{\circ}$ \\
\hline
\end{tabular}

Table 4.1: Grid synchronization values of the unit basis vector $\hat{o}$ and mno angles for balanced voltage condition at $400 \mathrm{~ms}$ and unbalanced voltage condition of Equation (4.15) at $500 \mathrm{~ms}$

The synchronization structure is an essential section of the mno instantaneous power control because it provides the instantaneous information needed to update the elements of the time-dependent mno transformation. By using this type of synchronization control, the voltage sourced converter does not require the traditional phase locked loop (PLL) for synchronization. Investigations have shown that traditional PLL dynamics might have undesirable impact on the performance of voltage sourced converters during imbalances when connected to weak AC systems [95, 96].

\subsection{3 - The $d q$ mno and the Inverse $d q$ mno Implementation}

The synchronization control structure provides the mno angles $\theta_{a}(t), \theta_{b}(t)$, $\theta_{c}(t), \phi_{a}(t), \phi_{b}(t)$ and $\phi_{c}(t)$ necessary to update the elements of mno transformation matrix $M_{m n o}(t)$, which allows the mno instantaneous voltage $\vec{v}_{m n o}(t)$ components to be instantaneously updated. Subsequently, the $d q$ mno voltage components $\vec{v}_{m n o}^{d q}(t)$ are 
obtained by the direct-quadrature mno matrix $M_{d q}$. This set of six sequence components allows the parametric visualization of the voltage in the three-dimensional frame (Section

\subsection{5, Chapter 3).}

The controller implementation of the direct-quadrature mno matrix $M_{d q}$ uses the SOGI-QSG structure presented earlier. The sequence component $v_{m}^{d}(t)$ presents the same phase angle reference of the component $v_{m}(t)$, which in turn has the same phase reference of the voltage $v_{a}(t)$. The signals in quadrature lag the direct input by $\pi / 2 \mathrm{rad}$. Figure $4.15 \mathrm{a}$ illustrates the implementation of the mno transformation for the voltage.

The voltage components $\vec{v}_{m n o}^{d q}(t)$ are utilized by the instantaneous power controller block illustrated at Figure 4.8 to generate the six $d q$ mno current components references, with the aim of producing oscillation free active and reactive powers as ordered by the input vector $\vec{p}^{*}(t)$, following an specific power control strategy, which will be discussed in detail in the next section.

Once the $d q$ mno instantaneous current references are synthesized, it is necessary to return to the $a b c$ coordinate system using the inverse mno transformation. First, the $d q$ mno current components references are converted to mno components using Equations (3.49) and (3.50), and then to $a b c$ coordinates using Equations (3.13) and (3.14), as shown in Figure 4.15b. 
(a)
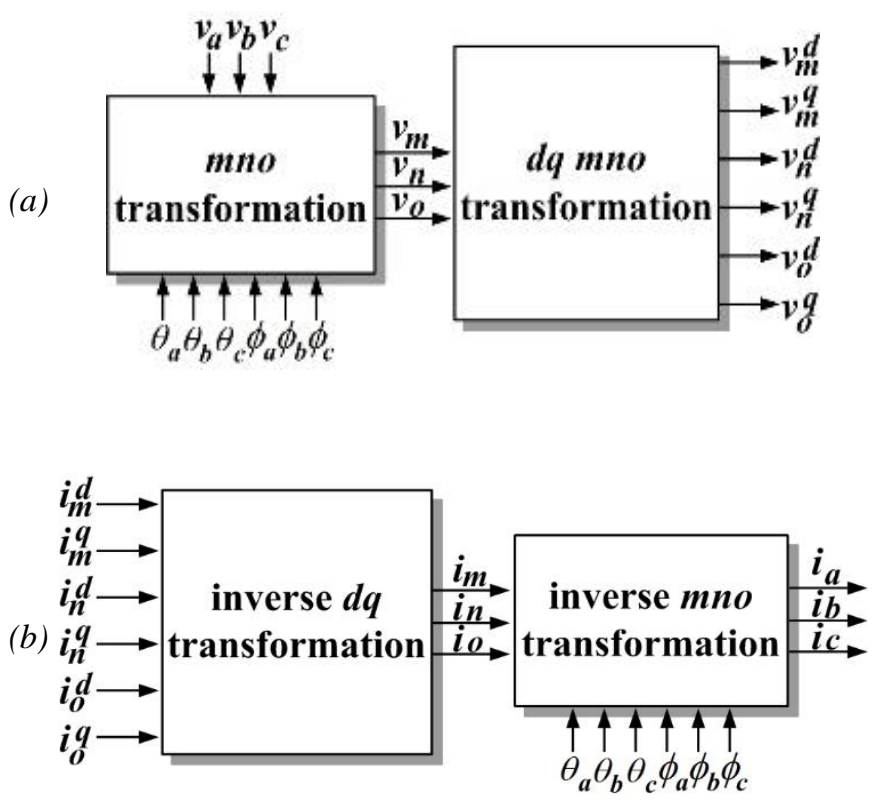

Figure 4.15: The dq mno implementation: (a) direct transformation, (b) inverse transformation

The mno transformation allows changing to a dimension where the mno instantaneous power components can be applied, as introduced in the Chapter 3. The inverse mno transformation returns to the $a b c$ coordinate system, providing the $a b c$ current references necessary to be established at each phase of the VSC, following a determined power strategy.

\subsection{4 - Power Control Strategies}

The mno instantaneous voltage components combined with the mno instantaneous current components are employed to calculate the instantaneous total power flowing at the three-phase transmission line, according to the Equation 3.74 introduced in the Chapter 3. Such mno power equation can be reversed, i.e. instead of calculating the power from the mno voltage and mno current components, the current components necessary to obtain the desired active and reactive powers can be calculated as with $V_{m n o}$ as in Equation (3.74) using the following expression: 


$$
\vec{i}_{m n o}^{d q^{*}}(t)=V_{m n o}^{-1}(t) \vec{p}^{*}(t)
$$

where the power order vector is represented by:

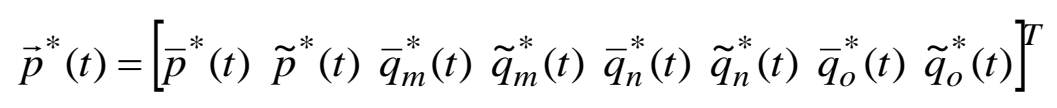

and $V_{m n o}^{-1}$ being the inverse matrix of the mno voltage matrix $V_{\text {mno }}$ given by:

$$
V_{m n o}=\left[\begin{array}{cccccc}
v_{m}^{d}(t) & v_{m}^{q}(t) & v_{n}^{d}(t) & v_{n}^{q}(t) & v_{o}^{d}(t) & v_{o}^{q}(t) \\
v_{m}^{q}(t) & v_{m}^{d}(t) & v_{n}^{q}(t) & v_{n}^{d}(t) & v_{o}^{q}(t) & v_{o}^{d}(t) \\
0 & 0 & -v_{o}^{d}(t) & -v_{o}^{q}(t) & v_{n}^{d}(t) & v_{n}^{q}(t) \\
0 & 0 & -v_{o}^{q}(t) & -v_{o}^{d}(t) & v_{n}^{q}(t) & v_{n}^{d}(t) \\
v_{o}^{d}(t) & v_{o}^{q}(t) & 0 & 0 & -v_{m}^{d}(t) & -v_{m}^{q}(t) \\
v_{o}^{q}(t) & v_{o}^{d}(t) & 0 & 0 & -v_{m}^{q}(t) & -v_{m}^{d}(t) \\
-v_{n}^{d}(t) & -v_{n}^{q}(t) & v_{m}^{d}(t) & v_{m}^{q}(t) & 0 & 0 \\
-v_{n}^{q}(t) & -v_{n}^{d}(t) & v_{m}^{q}(t) & v_{m}^{d}(t) & 0 & 0
\end{array}\right]
$$

and the generated reference current vector is represented by:

$$
\vec{i}_{m n o}^{d q^{*}}(t)=\left[i_{m}^{d^{*}}(t) i_{m}^{q^{*}}(t) i_{n}^{d^{*}}(t) i_{n}^{q^{*}}(t) i_{o}^{d^{*}}(t) i_{o}^{q^{*}}(t)\right]^{T}
$$

As the main objective is to maintain constant instantaneous power flow, the active and reactive powers oscillating terms in (4.20) are set to zero, providing a power order vector as:

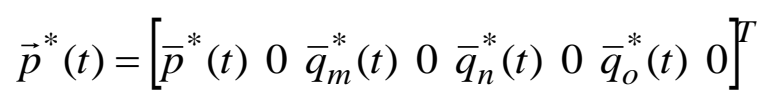

Considering ripple elimination, the constant term $\bar{p}^{*}(t)$ of the mno instantaneous power components is set equal to the desired active power order. For the instantaneous reactive power, which is represented by the magnitude of the vector $\vec{q}(t)$ as explained at Section 3.3 (Chapter 3$)$, the oscillating terms $\tilde{q}_{m}^{*}(t), \tilde{q}_{n}^{*}(t)$ and $\tilde{q}_{o}^{*}(t)$ are set equal to zero, 
and the net reactive power magnitude becomes $\sqrt{\left[\bar{q}_{m}^{*}(t)\right]^{2}+\left[\bar{q}_{n}^{*}(t)\right]^{2}+\left[\bar{q}_{o}^{*}(t)\right]^{2}}$, which may provide possible different choices in the selection of the constant reactive power components $\bar{q}_{m}^{*}(t), \bar{q}_{n}^{*}(t)$ and $\bar{q}_{o}^{*}(t)$.

In this research, the $\bar{q}_{m}^{*}(t)$ and $\bar{q}_{n}^{*}(t)$ reactive power orders are set equal to zero; consequently, the reactive power term $\bar{q}_{o}^{*}(t)$ is set equal to the desired reactive power order. This strategy is tantamount to make the orientation of the reactive power vector parallel to the instantaneous unit vector $\hat{o}(t)$ of the mno reference frame.

It can be seen from (4.16) that such condition forces the $\hat{o}$ current components $i_{o}^{d^{*}}(t)$ and $i_{o}^{q^{*}}(t)$ to be zero in the adjustable mno reference frame. Therefore, the current locus lies in the time-dependent $m n$ plane, just like the voltage, i.e. both voltage and current present only $m n$ components different than zero in the mno reference frame, even though zero sequence components are not zero in the $\alpha \beta 0$ stationary basis. The $m n$ components represent positive, negative and zero sequence content in a plane, similarly to the symmetrical components planar representation proposed by Fortescue [32].

This power control strategy provides four degrees of freedom related to four components of the $d q$ mno synthesized currents $i_{m}^{d}(t), i_{m}^{q}(t), i_{n}^{d}(t)$ and $i_{n}^{q}(t)$ to accomplish the total power ordered $\bar{p}^{*}(t), \tilde{p}^{*}(t), \bar{q}_{o}^{*}(t)$ and $\tilde{q}_{o}^{*}(t)$ under voltage scenarios which may include positive, negative and zero sequences represented by $v_{m}^{d}(t) v_{m}^{q}(t) v_{n}^{d}(t)$ and $v_{n}^{q}(t)$

Considering operation with the unbalanced voltage condition of Equation (4.15) characterized by positive, negative and zero sequences, active and reactive power orders 
respectively at $1.0 \mathrm{pu}$ and $0.25 \mathrm{pu}$ were given, based on the power strategy previously described.

Figure 4.16 shows results related to the unbalanced voltage of Equation (4.15): the mno voltage components; the $d q$ mno voltage components; as well as the $\alpha \beta 0$ voltage components for comparison with the mno voltage components, showing the zero sequence content.

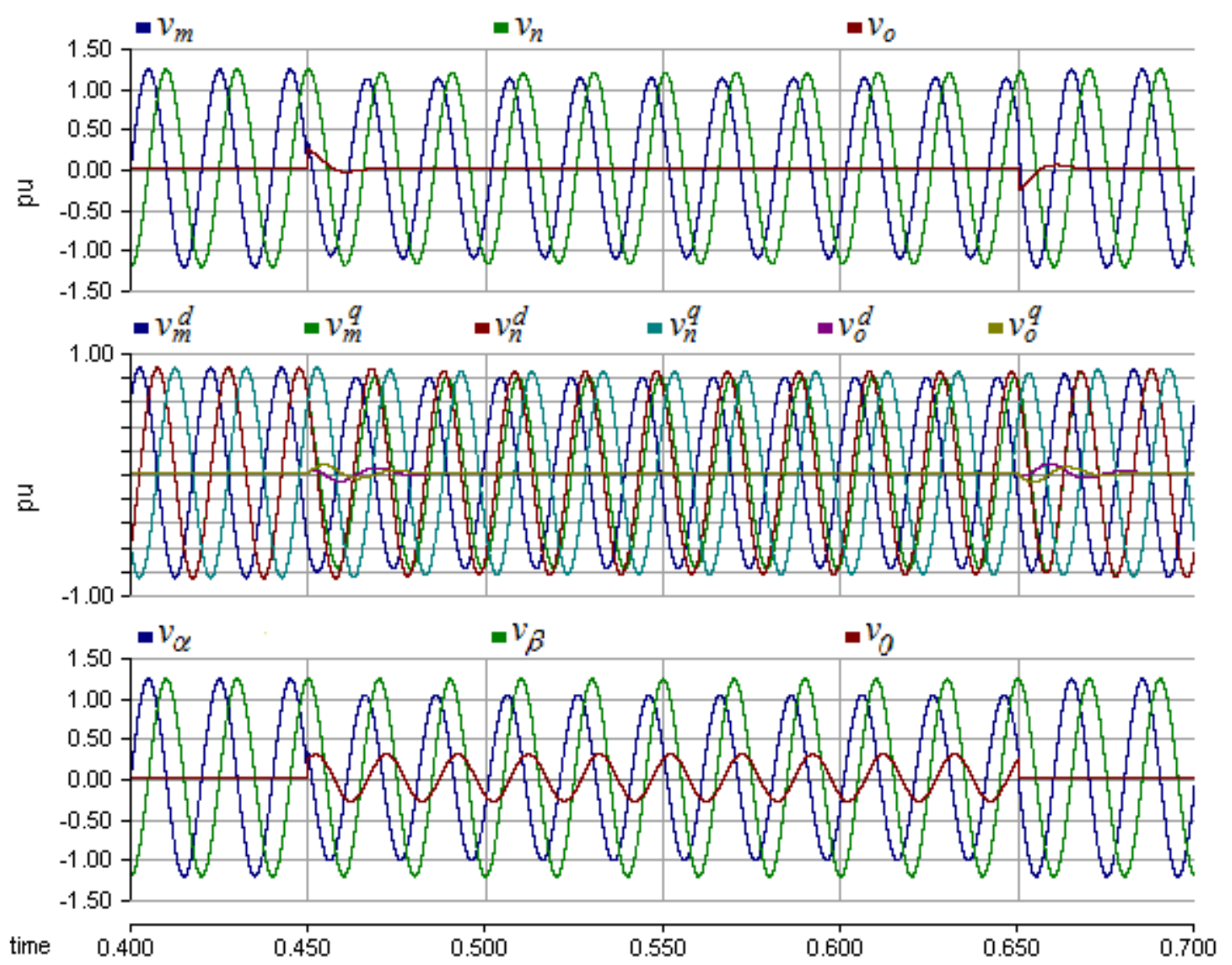

Figure 4.16: Voltage components of the mno instantaneous current components control for scenario of

Equation (4.15): mno voltage components, dq mno voltage components, $\alpha \beta 0$ voltage components

Figure 4.17 illustrates results related to the instantaneous power and current: the instantaneous active and reactive powers under the unbalanced voltage; the synthesized $d q$ mno current components; the respective mno components to achieve the oscillation free 
active and reactive powers as ordered; the equivalent generated reference current in the $a b c$ domain; as well as the $\alpha \beta 0$ current components for comparison with the mno current components and to illustrate the zero sequence current content.

It is evident that even during the unbalanced voltage condition from $450 \mathrm{~ms}$ to $650 \mathrm{~ms}$, the active and reactive powers remain essentially constant due to the transient controlled injection of the synthesized currents components of Figure 4.17. Note the $a b c$ currents are distorted during this transient condition. This is a tradeoff for maintaining the total active and reactive powers constant.

Once the necessary reference currents are evaluated, the next section presents the design of the controller which compares the reference current components with the measured current components, regulating the $\mathrm{AC}$ side converter voltage required to establish the reference current and, consequently, establish the desired power flow. 

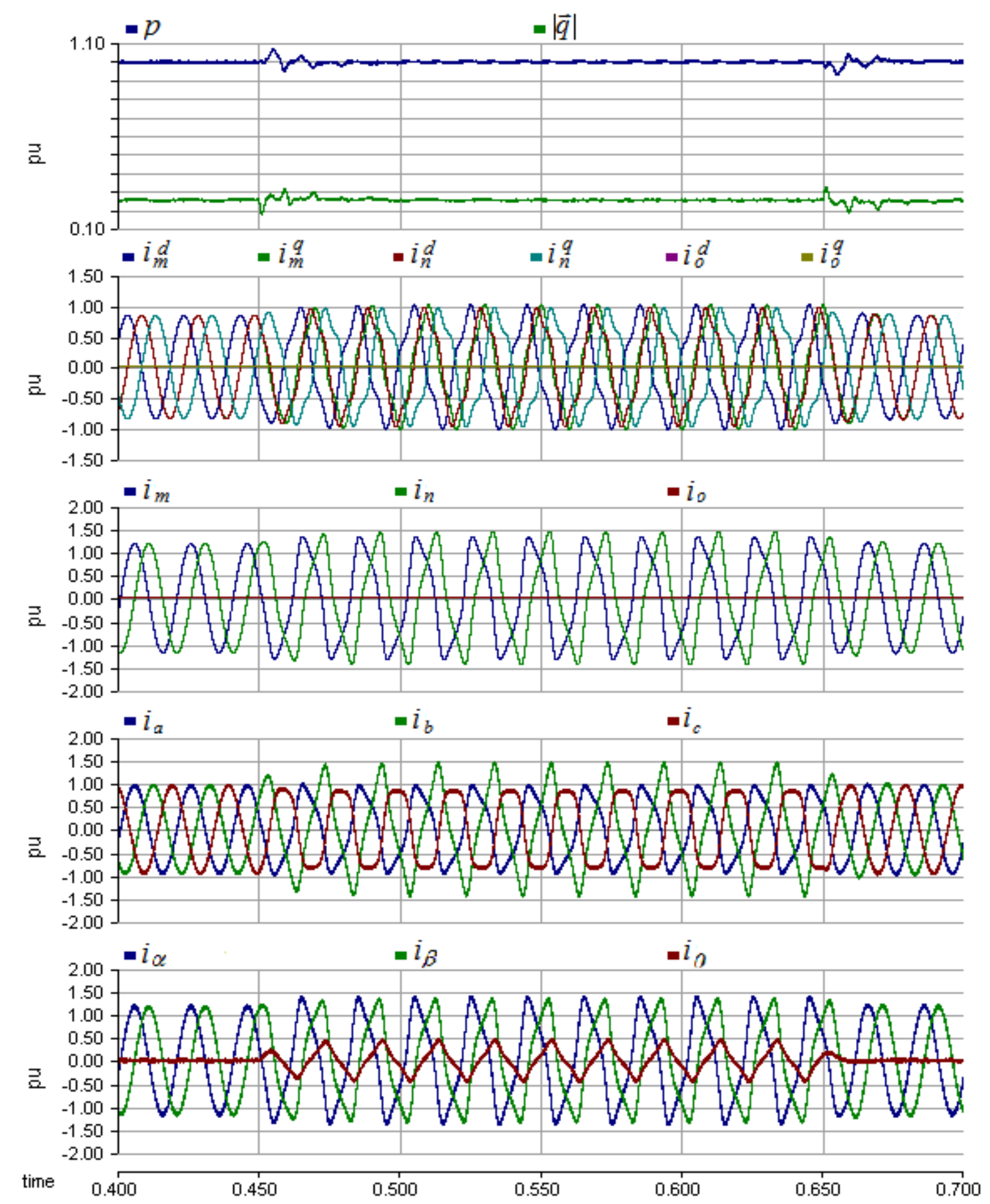

Figure 4.17: Instantaneous power and current components of the mno instantaneous current components control for scenario of Equation (4.15): instantaneous active and reactive powers, dq mno current components, mno current components, abc measured current, $\alpha \beta 0$ current components 


\subsection{5 - The Hysteresis Current Controller Implementation}

The current controller is implemented through a current reference PWM controller, also known as hysteresis controller, bang-bang controller or on-off controller, which is a feedback structure that switches between two states. This control compares the current references with the current measurements flowing at the point of common coupling to control the current on each phase.

The principle of hysteresis current control is quite straightforward: hysteresis comparators are used to impose a dead band or hysteresis around the reference current $i_{a}^{*}(t)$. The current control implementation employed $\pm 0.1 \mathrm{~A}$ band from this reference signal. Figure 4.18 illustrates these band limiting signals surrounding the reference current for phase $a$.

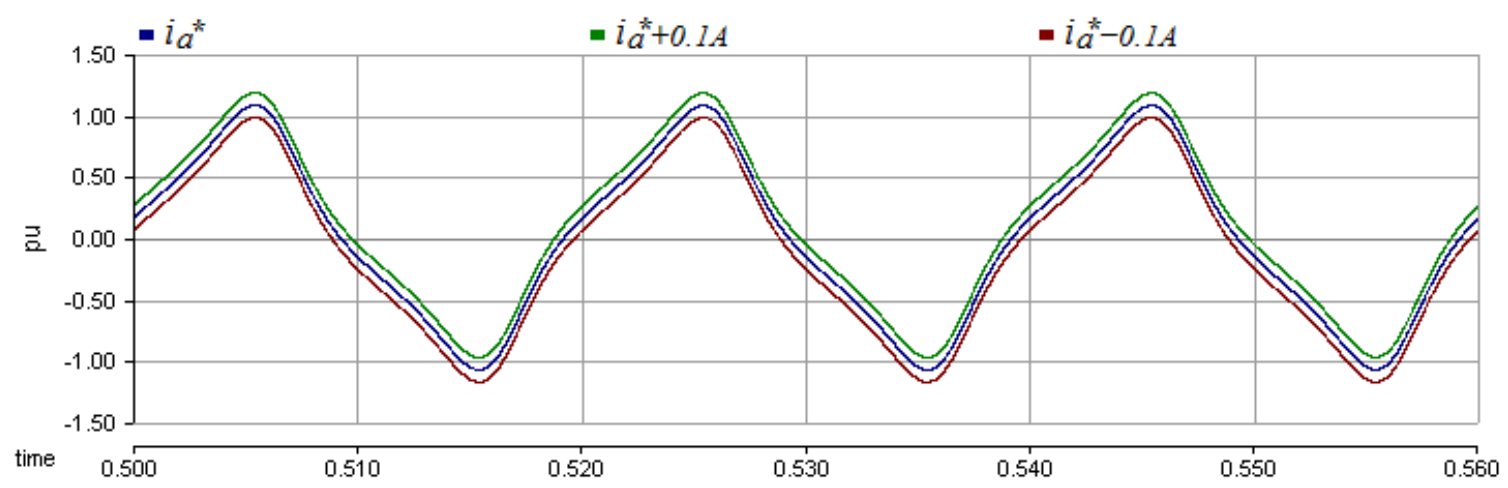

Figure 4.18: Current reference and dead band limiting signals

The switching frequency is high and varies during the fundamental period, as it can be seen in Figure 4.19, which represents an expanded view of a $10 \mathrm{~ms}$ section of Figure 4.18, including the actual measured current at phase $a$. The hysteresis bands are fixed over the fundamental period and the controller output oscillates between the limits imposed by the band limiting signals. 


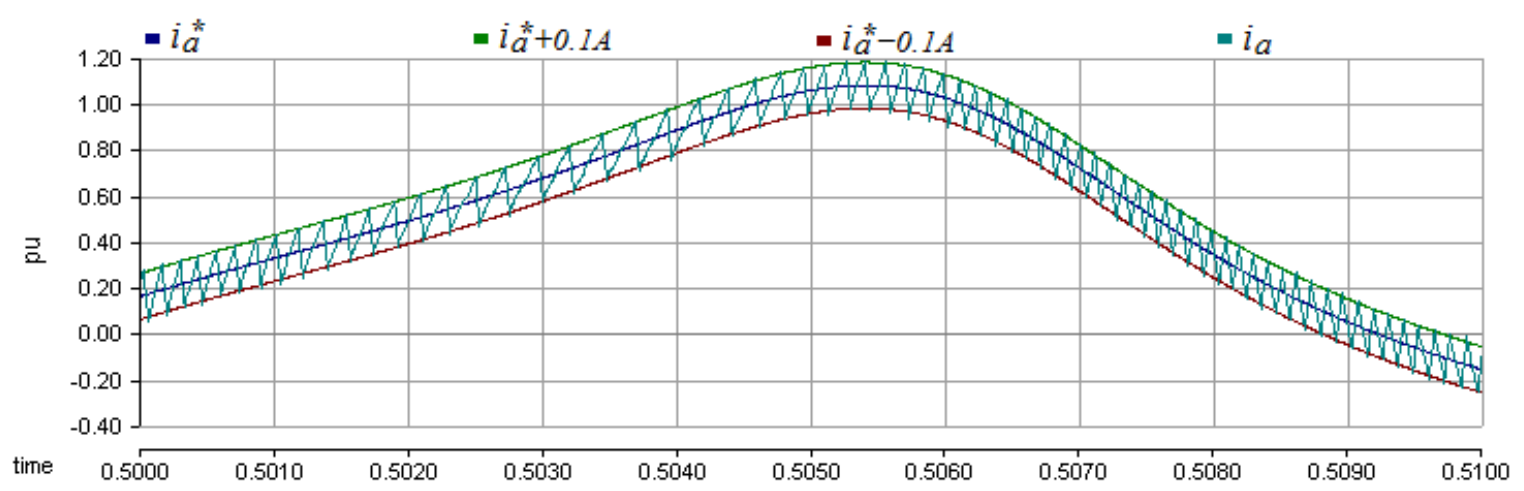

Figure 4.19: The hysteresis controlled current

The pulses are generated every time the controller output hits one signal of the band.

Figure 4.20 illustrates the PWM pulse signals generated for one of the switches of the converter bridge.

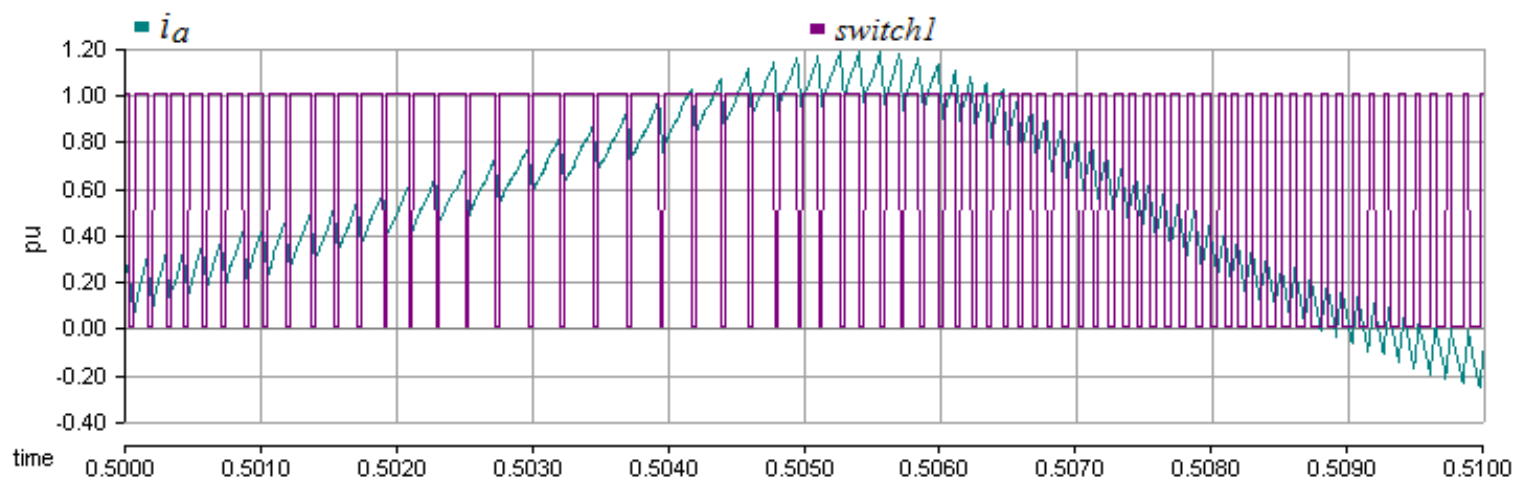

Figure 4.20: The hysteresis control and the PWM pulses

The advantage of the hysteresis control scheme is that it provides excellent dynamic performance because it acts quickly. The previous graphs of Figures 4.18, 4.19 and 4.20 show part of the current at the phase $a$ to illustrate the hysteresis control method, which is applied to each one of the three-phase current references, generating the required pulses for the converter switching cells as illustrated in Figure 4.20. Also, the current harmonics (in balanced steady-state) are small, thus reducing the necessity of an expensive low-pass filter. The disadvantage, however, is that the technique produces unpredictable high frequency components due to the switching instead of fixed frequency components as in a 
conventional SPWM. These high order frequencies can be filtered by the link filter between the converter and the point of common coupling.

This design provides a comprehensive understanding of the dynamic performance of the mno instantaneous power components control for grid connected voltage sourced converters during unbalanced AC conditions.

\subsection{6 - Unbalanced and Balanced Application Scenarios}

The mno instantaneous current components control was previously described using the unbalanced voltage sag at 0.8 pu concomitantly with a phase shift of $\pi / 6 \mathrm{rad}$ at phase $a$ (Equation 4.15), as an illustrative numerical example. This section presents results for three other scenarios, using the electrical system previously introduced: a two-phase voltage sag in phase $b$ and phase $c$, both at $0.7 \mathrm{pu}$ (unbalanced); a more severe single-phase voltage sag in phase $b$ at 0.2 pu (unbalanced); and a three-phase voltage sag at 0.8 pu in phases $a, b$ and $c$ (balanced).

The first case is characterized by the following $a b c$ voltages:

$$
\left\{\begin{array}{l}
v_{a}(t)=\cos (\omega t) \\
v_{b}(t)=0.7 \cos (\omega t-2 \pi / 3) \\
v_{c}(t)=0.7 \cos (\omega t+2 \pi / 3)
\end{array}\right.
$$

Figure 4.21 shows the results related to the scenario of Equation (4.21): the $a b c$ measured voltage; the mno pitch angles; the mno yaw angles; the mno voltage components and the correspondent $d q$ mno voltage components; as well as the $\alpha \beta 0$ voltage components. The unbalanced transient is simulated for 10 cycles, from $450 \mathrm{~ms}$ to $650 \mathrm{~ms}$. The mno instantaneous power control is able to synchronize the six mno angles within approximately 
one cycle. These angles allow to update the orientation of the $m n$ plane and the elements of the mno matrix, evaluating the mno and the $d q$ mno voltage components, as shown in Figure 4.21. The $\alpha \beta 0$ voltage components are also presented for comparison with the mno components, illustrating zero sequence voltage content.

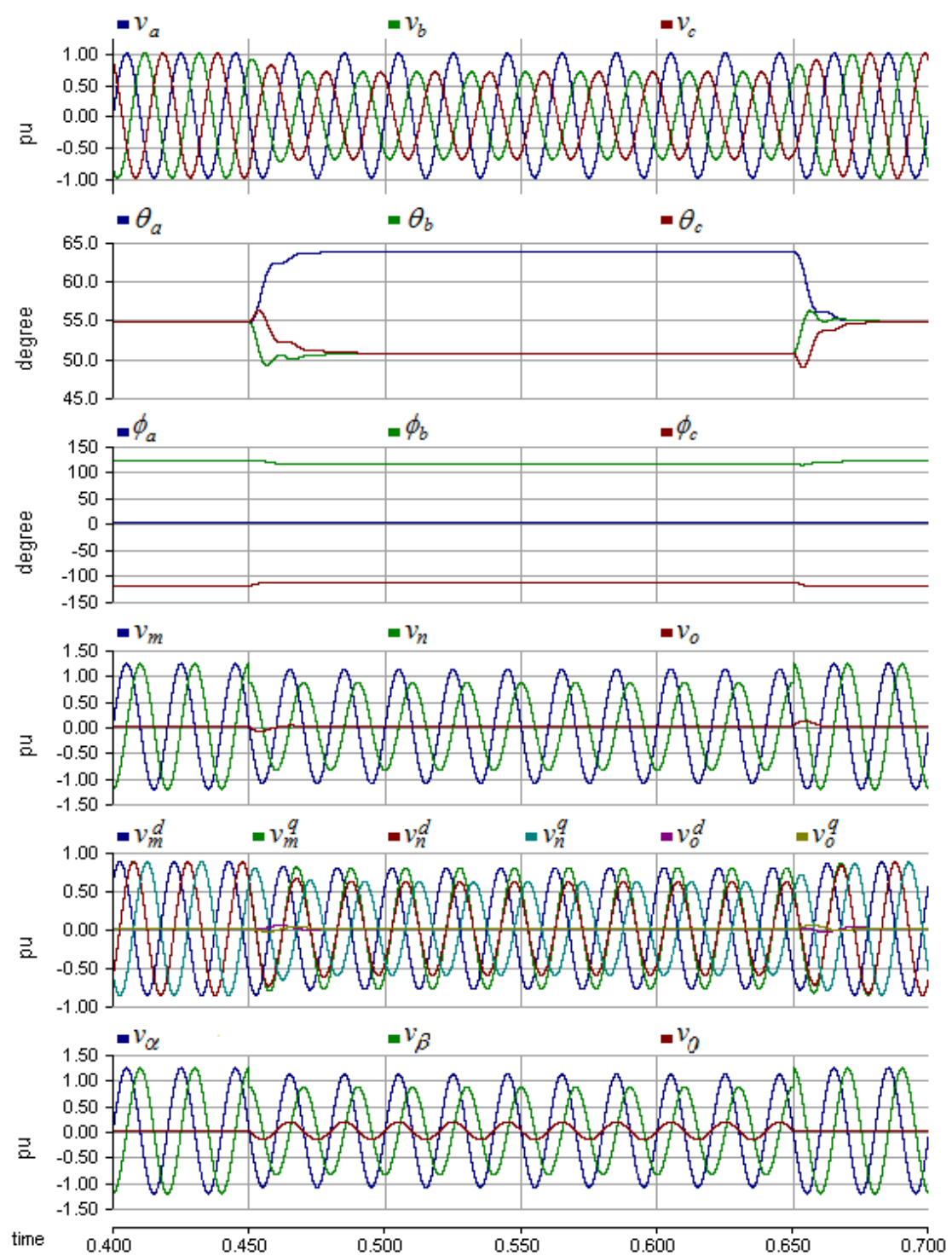

Figure 4.21: Voltage components and mno angles of the mno instantaneous current components control for scenario of Equation (4.21): abc measured voltage, mno pitch angles, mno yaw angles, mno voltage components, $d q$ mno voltage components, $\alpha \beta 0$ voltage components 
Figure 4.22 illustrates the instantaneous power and current components results for the same condition expressed by Equation (4.21): the instantaneous active and reactive power, the synthesized $d q$ mno current components reference, the correspondent mno current components, and the $a b c$ current measured at the point of common coupling, as well as the correspondent $\alpha \beta 0$ current components. The instantaneous active and reactive powers sustain constant respectively at $1.0 \mathrm{pu}$ and $0.25 \mathrm{pu}$.

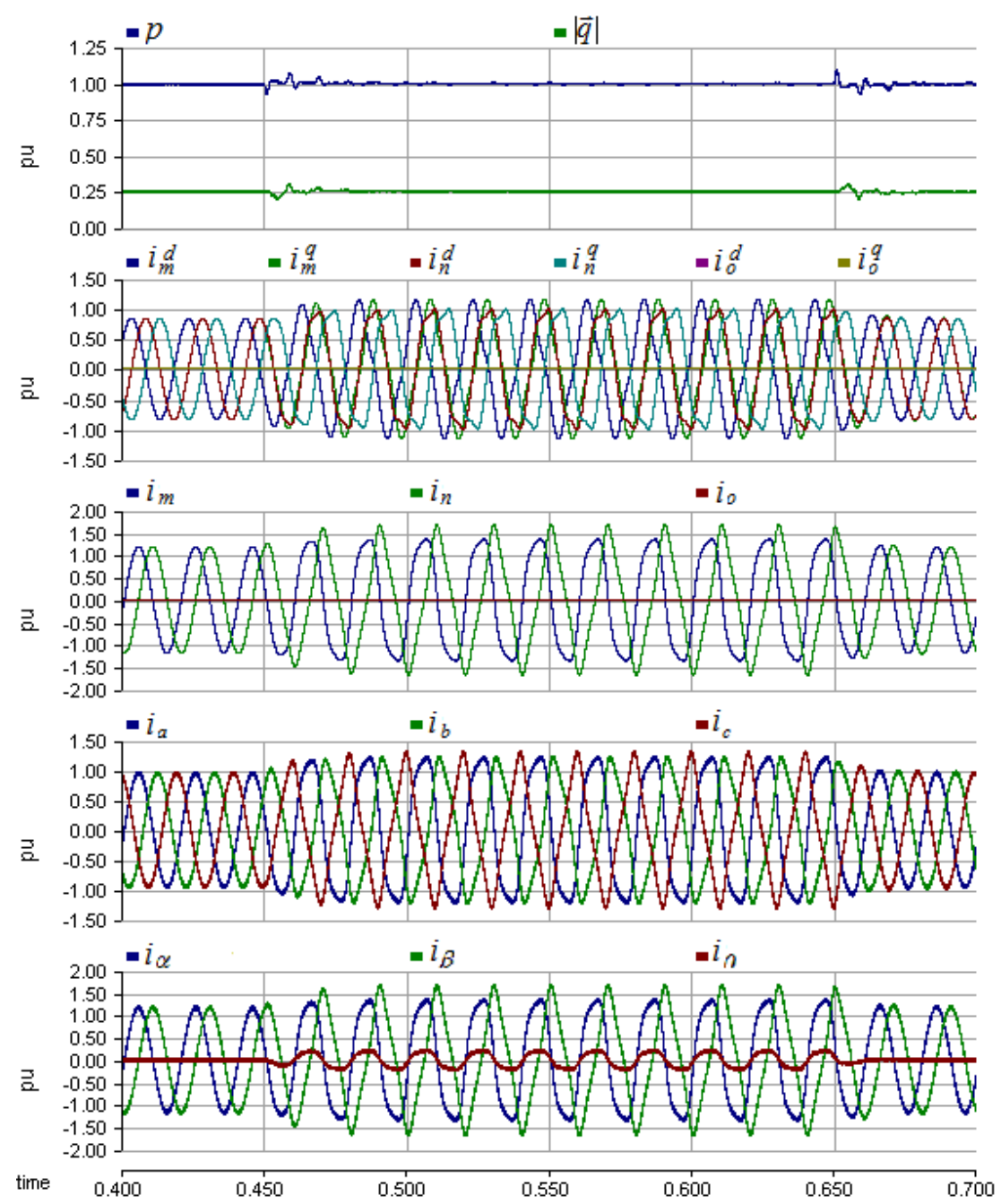

Figure 4.22: Instantaneous power and current components of the mno instantaneous current components control for scenario of Equation (4.21): instantaneous active and reactive powers, dq mno current components, mno current components, abc measured current, current components 
The second scenario is a severe single-phase voltage sag at 0.2 pu in phase $b$ :

$$
\left\{\begin{array}{l}
v_{a}(t)=\cos (\omega t) \\
v_{b}(t)=0.2 \cos (\omega t-2 \pi / 3) \\
v_{c}(t)=\cos (\omega t+2 \pi / 3)
\end{array}\right.
$$

Figure 4.23 illustrates waveforms for the voltage components as well as the mno angles, following the previous graphical presentation sequence of Figure 4.21.
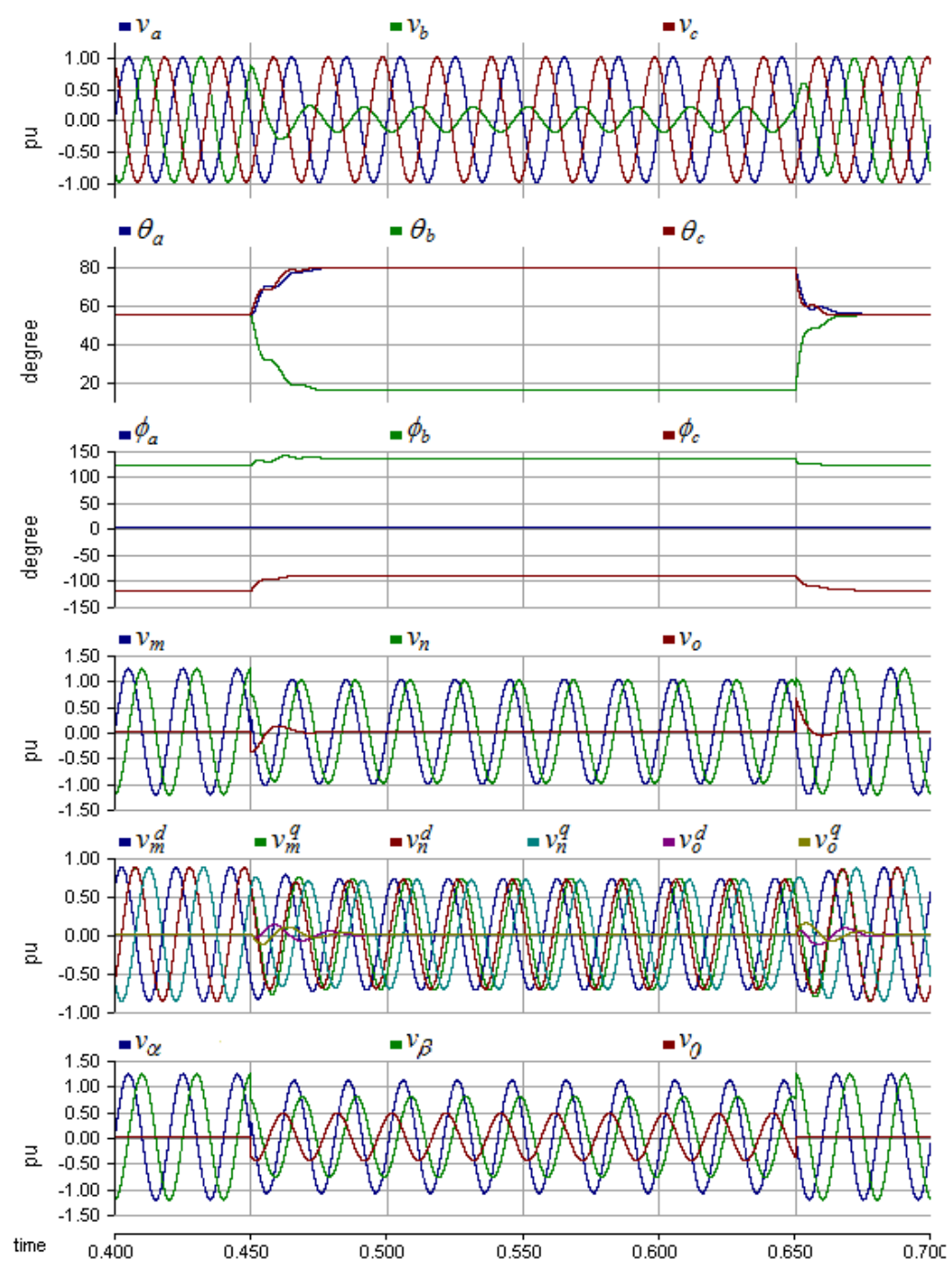

Figure 4.23: Voltage components and mno angles of the mno instantaneous current components control for scenario of Equation (4.22): abc measured voltage, mno pitch angles, mno yaw angles, mno voltage components, $d q$ mno voltage components, $\alpha \beta 0$ voltage components 
The mno synchronization control tracks the six mno angles within approximately one cycle, as illustrated in Figure 4.23. Note the increased zero sequence voltage component magnitude in the $\alpha \beta 0$ voltage components of Figure 4.23 when compared to Figure 4.21, characterized by the single-phase voltage dip at $0.2 \mathrm{pu}$.

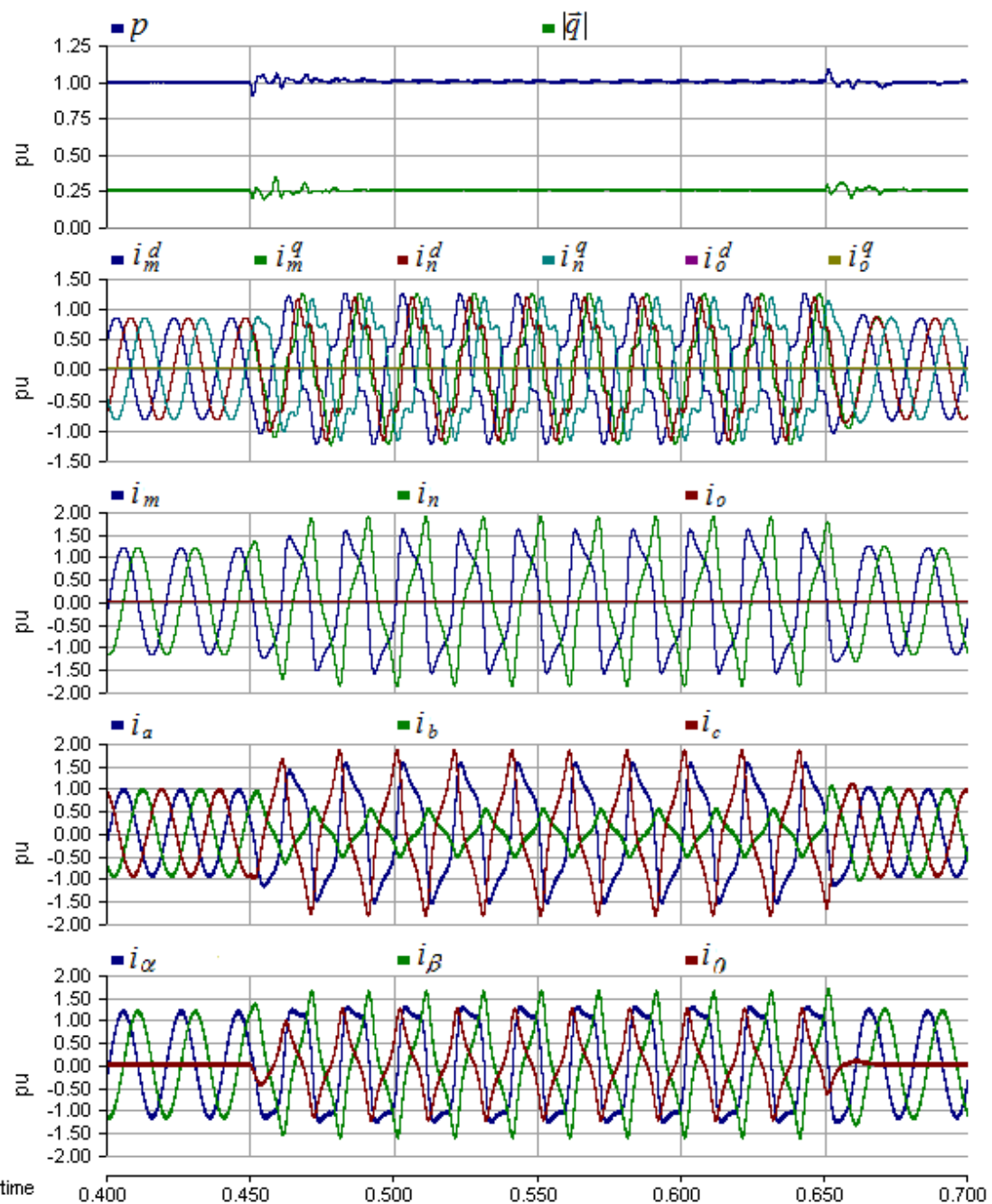

Figure 4.24: Instantaneous power and current components of the mno instantaneous current components control for scenario of Equation (4.22): instantaneous active and reactive powers, $d q$ mno current components, mno current components, abc measured current, $\alpha \beta 0$ current components

The mno, $d q$ mno and $a b c$ current components clearly presents higher harmonic distortion when compared to Figure 4.22. The $\alpha \beta 0$ current components illustrate zero sequence current content during the $200 \mathrm{~ms}$ transient. Note also phase $a$ and phase $c$ with 
elevated magnitude during the imbalance, which can be reduced by decreasing the power order during the imbalance, or by imposing limits for the maximum zero sequence content allowed, similarly as illustrated by Figure 3.5 (Chapter 3 ).

The third scenario shows a three-phase voltage sag at $0.8 \mathrm{pu}$ :

$$
\left\{\begin{array}{l}
v_{a}(t)=0.8 \cos (\omega t) \\
v_{b}(t)=0.8 \cos (\omega t-2 \pi / 3) \\
v_{c}(t)=0.8 \cos (\omega t+2 \pi / 3)
\end{array}\right.
$$

This scenario illustrates the general extended application of the proposed control approach, including balanced conditions. Figure 4.25 illustrates waveforms for the voltage components as well as the mno angles, following the previous graphical presentation sequence of Figures 4.21 and 4.23. The mno angles preserve equal values before and after the voltage sag, which are identical to Equations (3.38) and (3.39).

Note in Figure 4.25 that the results for the $\alpha \beta 0$ and for the mno voltage components are identical, as described in the Sections 3.2.4 (Chapter 3), illustrating the broader general application of the mno transformation and the $\alpha \beta 0$ transformation as an special case of the mno transformation. Figure 4.26 also shows the $\alpha \beta 0$ and the mno current components identical (balanced scenario), as well as the instantaneous active and reactive power constant. 


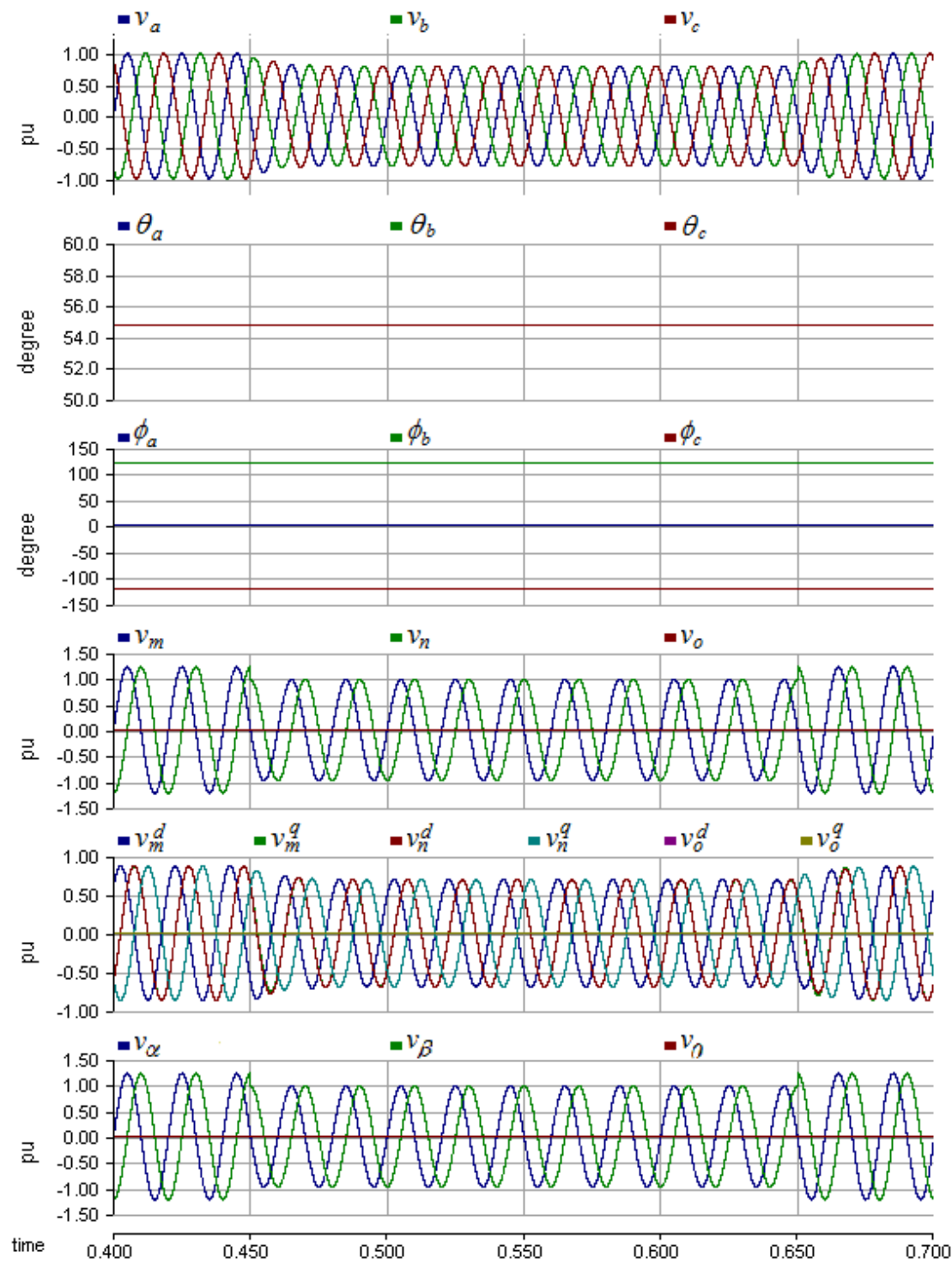

Figure 4.25: Voltage components and mno angles of the mno instantaneous current components control for scenario of Equation (4.23): abc measured voltage, mno pitch angles, mno yaw angles, mno voltage components, $d q$ mno voltage components, $\alpha \beta 0$ voltage components 


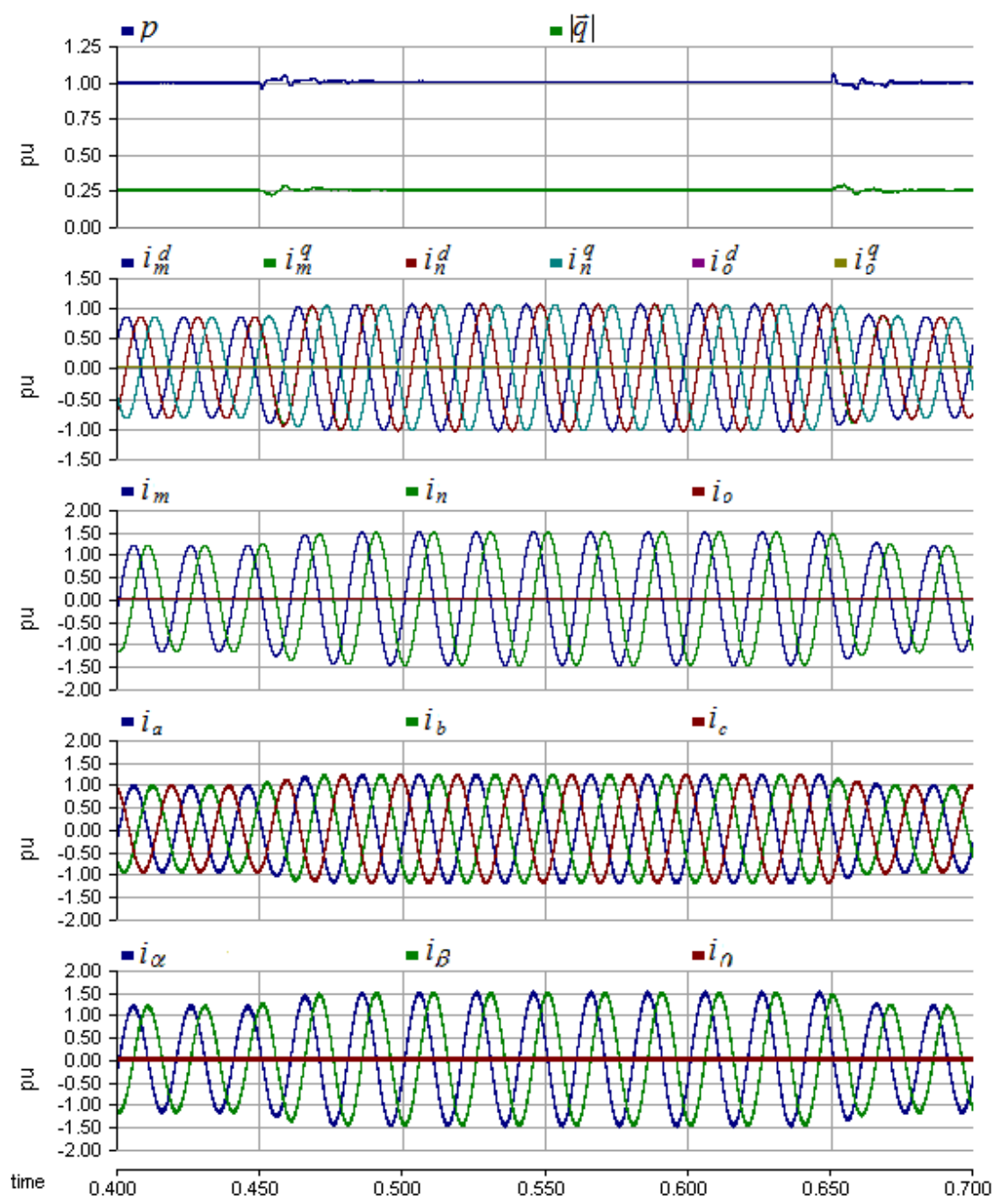

Figure 4.26: Instantaneous power and current components of the mno instantaneous current components

control for scenario of Equation (4.23): instantaneous active and reactive powers, dq mno current

components, mno current components, abc measured current, $\alpha \beta 0$ current components

For these three cases represented by Equations (4.21), (4.22) and (4.23), it is evident that even during the unbalanced voltage condition from $450 \mathrm{~ms}$ to $650 \mathrm{~ms}$, the active and reactive powers remain essentially constant due to the transient controlled injection of the synthesized currents components. Note the $a b c$ currents are distorted during this transient unbalanced condition, a tradeoff for maintaining the total active and reactive powers constant. 


\section{Chapter 5}

\section{Conclusion}

The research topics addressed in this thesis are summarized in the following sections, describing the main contributions and conclusions from the studies undertaken during the research and development of the theory proposed in the present work. Moreover, future tasks are proposed, with insights into potential new developments as a continuation of this thesis, as well as different possible applications.

\section{1 - Main Contributions and Conclusions}

The key contributions of the present doctorate research are:

- The thesis presents a comprehensive description of the most important past work in regards to vector transformations developed for analysis of unbalanced three-phase voltages and three-phase currents;

- It charts the evolution of the instantaneous power concepts for single-phase and three-phase AC systems, including their advantages and disadvantages for different balanced, unbalanced and distorted scenarios. The modern power theories available to deal with challenging adverse AC conditions were presented, highlighting the state-of-the-art 
of the $p-q$ theory and the modified $p-q$ theory, as a widely used robust tool when applied to power conditioning;

- $\quad$ The present work describes the problem of power oscillations when power is transferred in scenarios with unbalanced three-phase voltages and unbalanced threephase currents. This issue is more problematic with transients where zero sequence power is present, i.e. zero sequence voltage and zero sequence current. This is one of the reasons why zero sequence current is not welcome in most circuits, being frequently avoided, focusing the analyses on three-wire systems;

- When investigating power oscillations associated to zero sequence transients, a drawback is identified in the $p$ - $q$ theory, which describes the characteristics of the zero sequence power similarly to the instantaneous active power in single-phase circuits, with constant and oscillating terms concomitantly present. This theory states that "zero-sequence components should be avoided in three-phase systems because these cannot produce three-phase constant power". The $p$ - $q$ theory is attached to the stationary $\alpha \beta 0$ transformation, which in turn sparked investigations towards a new transformation.

- The thesis proposes a new vector transformation method (the mno transformation). It is a time-dependent adaptive transformation (not stationary like the $\alpha \beta 0$ transformation), which converts three-phase voltages and currents from the $a b c$ domain to a new mno domain. The mno transformation updates its elements instantaneously based on the system voltage dynamic conditions. The direct and inverse transformations are described with details;

- $\quad$ Based on the mno transformation, the mno instantaneous power equations are introduced as an innovative procedure to calculate the instantaneous power, applicable 
to balanced or unbalanced AC conditions, including positive, negative and zero sequence voltage and current components;

- An advantageous feature is recognised in the mno transformation and the mno instantaneous power equations, which allows the calculation of the instantaneous electric power in a particularly advantageous manner. It enables the decomposition of the total instantaneous power into constant and oscillating terms, especially for adverse AC scenarios with three independent voltages and three independent currents;

- Three-dimensional visualization methods are launched in order to track voltage and current signals in the mno reference frame introduced, including threedimensional vector representation of the reactive power, with its decomposition into constant and varying vectors, as well as the reactive power locus in three-dimensional cartesian reference frame;

- $\quad$ The mno power theory is summarized as an enhanced instantaneous power theory, improving previous concepts based on the $p$ - $q$ theory. It presents simple but powerful techniques which expands the related power engineering knowledge towards the solution of power oscillations in unbalanced three-phase circuits with positive, negative and zero sequence power components;

- $\quad$ The mno power theory opens up the possibility of having constant active and reactive power transmission in voltage sourced converter (VSC) during unbalanced conditions, including operation under challenging scenarios with zero sequence power;

- A control algorithm was developed and tested for different three-phase voltage dynamic conditions to map the mno components from $a b c$ coordinates and viceversa; 
- The new power control method for grid connected voltage sourced converters was introduced, which generates current orders for the VSC so that the oscillatory components of active and reactive powers becomes zero. As the mno transformation allows independent control of the constant and oscillatory components of the instantaneous power, signals for the mno currents can be synthesised to accomplish such task. The $a b c$ phase current orders for the voltage sourced converter can then be calculated by the inverse mno transformation. A hysteresis controller was used in the VSC to create the actual currents from these orders;

- The viability of the control method was confirmed using electromagnetic transient simulation for a number of scenarios (single-phase voltage sag, single-phase phase shift, two-phase voltage sag, three-phase voltage reduction, etc). The control performance was satisfactory and the constancy in the instantaneous power was achieved for the grid connected voltage sourced converters under such unbalanced conditions, including operation with zero sequence voltage and current;

- One disadvantage of the proposed method was identified related to the harmonic content of the controlled current, depending on how severe is the transient, such as the single-phase voltage dip at $0.2 \mathrm{pu}$. This was the tradeoff in order to keep the power constant. Furthermore, this is likely to be a transient phenomenon under unbalanced conditions only. In several situations, such as power transmission from a remote wind power plant, it is important to maintain constancy of power, and small duration distortions could be tolerated; 
- The research presented in this thesis was submitted, reviewed and published by the IEEE Transactions on Power Electronics. An electronic version of the paper is available for download at the IEEE Digital Library as:

A. A. MONTANARI and A. M. GOLE, Enhanced Instantaneous Power Theory for Control of Grid Connected Voltage Sourced Converters Under Unbalanced Conditions, in IEEE Transactions on Power Electronics, vol. 32, no. 8, pp. 66526660, Aug. 2017.

\section{2 - Future Work}

The following sections propose future tasks possible to be developed as a continuation of this doctorate thesis.

\subsection{1 - Synchronous mno Transformation}

Research can be conducted to create a synchronous version of mno transformation. The idea is to establish rotating references for the $m n$ components at the adaptive $m n$ plane, which is a time-dependent plane. The procedure aims to be similar to the relation between the two-dimensional $\alpha \beta$ and $d q$ transformations, incorporating advantages of the mno power components. A synchronous mno transformation will permit the $d q m n$ components to become constant values in the synchronous domain, allowing the mno current components to be regulated by proportional integral controllers. 


\subsection{2 - Reactive Power Control Strategies}

The instantaneous total reactive power is defined as the magnitude of the vector created by the vector product between the voltage and current instantaneous vectors. For the inverse operation described in Section 4.2.4, i.e. to generate current references based on the grid voltage and power orders, the same reactive power order can be given by an infinite combination of vector components which may present equal instantaneous total reactive power magnitude. Thus, other reactive power strategies might be possible to be investigated.

\subsection{3 - Control Improvements and Performance Evaluation}

An important extension would be to make the mno current control method adaptive to frequency changes. Distorted voltages scenarios can be addressed using M-SOGI-QSG control structures (Multiple Second Order Generalized Integrator Quadrature Signal Generator) tuned at different frequencies. Then, multiple resonant controllers structures tuned in different frequencies can be used in parallel to control the current components. This will also allow the detection of harmonic components, exploring other possible functionalities of the converter in regards to active filtering, resonance damping or stability issues.

The system performance can be tested for different adverse AC scenarios, considering the voltage sourced converter operation for common unbalanced transient conditions such as: voltage sags, overvoltages and voltage phase shift; with distortion. The influence of the ground impedance, in the absence of the neutral wire, and other transformer connections (e.g. the zig-zag) could also be investigated. 


\subsection{4 - VSC Application Cases}

Two breakthrough technologies are shaping the DC transmission developments: the Modular Multilevel Voltage Sourced Converter and the Multiterminal VSC-HVDC Systems (DC Grids). Thus, final application cases may be considered to evaluate the mno instantaneous power control strategies for these systems.

The MMC-VSC Cascade H-bridge wye connected can been explored. For the Multiterminal VSC-HVDC, the proposal control might help the DC grid high level controls, such as the margin or the droop control, reducing the impact of power oscillations in the DC network. The method might also aid to clear DC faults, as the zero sequence current is under control, forcing the controlled component to be zero. 


\section{References}

[1] PLATO; The Republic, 514a-520a, 380 BC.

[2] MCNICHOL, T; AC/DC: The Savage Tale of the First Standards War, Jossey-Bass, 2013.

[3] HALACSY, A.; VON FUCHS, G.; Transformer Invented 75 Years Ago, Transactions of the American Institute of Electrical Engineers, v. 80, i. 3, p. 121-125, 1961.

[4] LAMM, U.; Mercury-Arc Valves for High-Voltage DC Transmission, IEEE Proceedings, v. 111, i. 10, p. 1747-1753, 1964.

[5] BRANDT, D.; MCKAY, I.; RASHWAN, M.; RANADE, S.; Paralleling and Deparalleling Tests on Nelson River HVDC Bipoles 1 and 2, IEEE Transactions on Power Apparatus and Systems, v. 103 ,i. 4, p. 762-770, 1984.

[6] HAMMONS, T.; WOODFORD, D.; LOUGHTAN, J.; CHAMIA, M.; DONAHOE, J.; POVH, D.; BISEWSKI, B.; LONG, W.; Role of HVDC Transmission in Future Energy Development, IEEE Power Engineering Review, v. 20, i. 2, p. 10-25, 2000.

[7] IRWIN, G.; ISAACS, A.; WOODFORD, D.; Simulation Requirements for the Atlantic Wind Multi-Terminal VSC Offshore Wind Project, Transmission and Distribution Conference and Exposition, p. 1-4, 2012.

[8] FAIRLEY, P.; DC Versus AC: The Second War of Currents has already begun, IEEE Power and Energy Magazine, v. 10, i. 6, p. 103-104, 2012. 
[9] WANG, P.; GOEL, L.; LIU, X.; CHOO, F.; Harmonizing AC and DC: A Hybrid AC/DC Future Grid Solution, IEEE Power and Energy Magazine, v. 11, i. 3, p. 76-83, 2013.

[10] LIU, Y.; CHEN, Z.; A Flexible Power Control Method of VSC-HVDC Link for the Enhancement of Effective Short-Circuit Ratio in a Hybrid Multi-Infeed HVDC System, IEEE Transactions on Power Systems, v. 28, i. 2, p. 1568-1581, 2013.

[11] MEERE, R. ; O'MALLEY, M. ; KEANE, A.; VSC-HVDC Link to Support Voltage and Frequency Fluctuations for Variable Speed Wind Turbines for Grid Connection, International Conference and Exhibition on Innovative Smart Grid Technologies, p. 1-5, 2012.

[12] SINGH, B.; JAYAPRAKASH, P.; KOTHARI, D.; Isolated H-bridge VSC Based 3phase 4-wire DSTATCOM For Power Quality Improvement, International Conference on Sustainable Energy Technologies, p. 366-371, 2008.

[13] ADAM, G.; AHMED, K.; FINNEY, S.; WILLIAMS, B.; AC Fault Ride-Through Capability of VSC-HVDC Transmission Systems, IEEE Energy Conversion Congress and Exposition, p. 3739-3745, 2010.

[14] BEDDARD, A.; BARNES, M.; AC Fault Ride-Through of MMC VSC-HVDC Systems, International Conference on Power Electronics, Machines and Drives, p. 1-6, 2014.

[15] RODRIGUEZ, P.; LUNA, A.; CIOBOTARU, M.; TEODORESCU, R.; BLAABJERG, F.; Advanced Grid Synchronization System for Power Converters under Unbalanced and Distorted Operating Conditions, IEEE Annual Conference on Industrial Electronics. 5173-5178, 2006. 
[16] CHAUDHARY, S.; TEODORESCU, R.; RODRIGUEZ, P.; KJAER, P.; GOLE, A.; Negative Sequence Current Control in Wind Power Plants With VSC-HVDC Connection, IEEE Transactions on Sustainable Energy, v. 3, i. 3, p. 535-544, 2012.

[17] RODRIGUEZ, P.; TIMBUS, A.; TEODORESCU, R.; LISERRE, M.; BLAABJERG, F.; Flexible Active Power Control of Distributed Power Generation Systems During Grid Faults, IEEE Transactions on Industrial Electronics, v. 54, i. 5, p. 2583-2592, 2007.

[18] ZHANG, J.; CHEN, H.; PAN, W.; WANG, C.; VSC-HVDC Control Under Unbalanced Supply Conditions, IEEE Power Engineering Society General Meeting, $\quad$ p. $1-6,2007$.

[19] DU, C.; SANNINO, A.; BOLLEN, M.; Analysis of Response of VSC-Based HVDC to Unbalanced Faults with Different Control Systems, IEEE Transmission and Distribution Conference and Exhibition: Asia and Pacific, p. 1-6, 2005.

[20] LIANXIANG, T.; BOON-TECK, O.; Managing Zero Sequence in Voltage Source Converter, Industry Applications Conference Annual Meeting, v. 2, p. 795-802, 2002.

[21] MUNCH, P.; LIU, S.; EBNER, G.; Multivariable Current Control of Modular Multilevel Converters with Disturbance Rejection and Harmonics Compensation, IEEE International Conference on Control Applications, p. 196-201, 2010.

[22] SORENSEN, R.; NEWTON, W.; Inherent Voltage Relations in $Y$ and Delta Connections, Transactions of the American Institute of Electrical Engineers, v. XXXIII, i. 1, p. 711-721, 1914.

[23] EDEN, T.; Relative Merits of $Y$ and Delta Connection for Alternators, Transactions of the American Institute of Electrical Engineers, v. XXXIII, i. 1, p. 803-806, 1914. 
[24] OWEN, E.; The Historical Development of Neutral Grounding Practices, Industry IEEE Applications Magazine, v. 3, i. 2, p. 10-20, 1997.

[25] IEEE STANDARD; IEEE Standard Requirements, Terminology, and Test Procedures for Neutral Grounding Devices, ANSI/IEEE, n. 32, 1972.

[26] GLOVER, J.; SARMA M.; OVERBYE, T.; Power System Analysis \& Design, Cengage Learning, 2011.

[27] GRAINGER, J.; STEVENSON, W.; Power System Analysis, McGraw-Hill, 1994.

[28] TESLA, N.; Electro-Magnetic Motor, United States Patent Office, Patent No. 381.968, 1888.

[29] FERRARIS, G.; Rotazioni Elettrodinamiche Prodotte per Mezzo di Correnti Alternate, Atti dell'Accademia della scienze, v. 23, i. 1, p. 360-363, 1888.

[30] STOKVIS, L.; Der Spannungsabfall des Synchronen Drehstromgenerators bei Symmetrischer Belastung, R. Oldenbourg, Munich, 1912.

[31] STOKVIS, L.; Analysis of Unbalanced Three-Phase Systems - Reactions in a Generator Carrying an Unbalanced Load Treated as Equivalent to Two Balanced Loads, Electrical World, v. 65, pp. 1111-1115, 1915.

[32] FORTESCUE, C.; Method of Symmetrical Coordinates Applied to the Solution of Polyphase Networks, Transactions of the American Institute of Electrical Engineers, v. 37, n. 2, p. 1027-1140, 1918.

[33] EVANS, R.; Finding Single-phase Short-circuit Currents on Calculating Boards, Electrical World, v. 85, p. 761-764, 1925. 
[34] BEKKU, S.; Calculation of Short-Circuit Ground Currents on Three-Phase Power Networks Using the Method of Symmetrical Coordinates, General Electric Review, v. 28, n. 7, p. 472-478, 1925.

[35] HEYDT, G.; VENKATA, S.; BALIJEPALli N.; High Impact Papers in Power Engineering 1900-1999, North American Power Symposium, p. 1-7, 2012.

[36] FURFARI, F.A.; BRITTAIN, J.; Charles Legeyt Fortescue and the Method of Symmetrical Components, IEEE Industry Applications Magazine, v. 8, i. 3, p. 7-9, 2002.

[37] LYON, W.; Transient Analysis of Alternating Current Machinery, Technology Press of The Massachusetts Institute of Technology, 1954.

[38] WHITE, D. C.; WOODSON H.; Electro Mechanical Energy Conversion, New York and Chapman \& Hall, John Wiley \& Sons, 1959.

[39] SILVA, S.; LOPES, B.; FILHO, J.; CAMPANA, R.; BOSVENTURA, W.; Performance Evaluation of PLL Algorithms for Single-Phase Grid-Connected Systems, IEEE Industry Applications Conference, v. 4, p 2259-2263, 2004.

[40] RADER, C.; JACHSON, L.; Approximating Noncausal IIR Digital Filters Having Arbitrary Poles, Including New Hilbert Transform Designs, Via Forward/Backward Block Recursion, IEEE Transactions on Circuits and Systems, p. 2779-2787, 2006.

[41] HAYKIN, S.; Adaptive Filter Theory, Upper Saddle River, Prentice Hall, 2002.

[42] LEWIS, W.; Short Circuit Currents on Grounded Neutral Systems, General Electric Review, p. 524-527, 1917.

[43] PARK, R.; SKEATS, W.; Circuit Breaker Recovery Voltages - Magnitudes and Rates of Rise, Transactions of the American Institute of Electrical Engineers, v. 50, i. 1, p. 204$238,1931$. 
[44] CLARKE, E.; WEYGANDT C.; CONCORDIA C.; Overvoltages Caused by Unbalanced Short Circuits Effect of Amortisseur Windings, Transactions of the American Institute of Electrical Engineers, v. 57, i. 8, p. 453-468, 1938.

[45] STANLEY, H.; An Analysis of the Induction Machine, Transactions of the American Institute of Electrical Engineers, v. 57, p. 751-757, 1938.

[46] CLARKE, E.; Problems Solved by Modified Symmetrical Components, General Electric Review, v. 41, i. 11, p. 488-494, 1938.

[47] TAHRI, F.; TAHRI, A.; ALRADADI, E. A.; DRAOU A.; Analysis and Control of Advanced Static VAR Compensator Based on the Theory of the Instantaneous Reactive Power, International Aegean Conference on Electrical Machines and Power Electronics, p. 840-845, 2007.

[48] CLARKE, E.; WEYGANDT C. N.; CONCORDIA C.; Overvoltages Caused by Unbalanced Short Circuits: Effect of Amortisseur Windings, v. 57, i. 3, pp. 453-468, 1938. [49] PARK, R.; Two-Reaction Theory of Synchronous Machines Generalized Method of Analysis - Part I, Transactions of the American Institute of Electrical Engineers, v. 48, i. 3, p. 716-727, 1929.

[50] AREERAK, K.; BOZHKO, S.; ASHER, G.; THOMAS, D.; DQ-Transformation Approach for Modelling and Stability Analysis of AC-DC Power System with Controlled PWM Rectifier and Constant Power Loads, EPE-PEMC Power Electronics and Motion Control Conference, p. 2049-2054, 2008.

[51] KERCHNER, R.; CORCORAN G.; Alternating Current Circuits, Wiley, 1938.

[52] LYON, W.; Reactive Power and Unbalanced Circuits, Electrical World, v. 75, n. 25, p. $1417-1420,1920$. 
[53] BUCHHOLZ, F.; Die Drehstrom-Scheinleistung bei Ungleichmabiger Belastung der Drei Zweige, Licht und Kraft, Zeitschrift fiir elekt. Energie-Nutzung, n. 2, p. 9-11, 1922. [54] BUDEANU, C.; Puissances Reactives et Fictives, Instytut Romain de l'Energie, n. 2, 1927.

[55] CZARNECKI, L.; What is wrong with The Budeanu Concept of Reactive and Distortion Power and why it should be abandoned, IEEE Transactions Instrumentation and Measurement, v. 36, i. 3, p. 834-837, 1987.

[56] IEEE STANDARD; The IEEE Standard Dictionary of Electrical and Electronics Terms, IEEE, 1997.

[57] FRYZE, S.; Wirk-, Blind- und Scheinleistung in Elektrischen Stromkreisen mit Nichtsinusförmigem Verlauf von Strom und Spannung, Elektrotechnische Zeitschrift, $\quad$ v. 53, n. 25, p. 596-599, 625-627, 700-702, 1932.

[58] DEPENBROCK, M.; The FBD-Method, a Generally Applicable Tool for Analyzing Power Relations, IEEE Transactions on Power Systems, v. 8, n. 2, 381-387, 1993.

[59] CZARNECKI, L.; Orthogonal Components of Linear Loads Current, Scientific Letters Elektryka, n. 86, p. 5-17, 1983.

[60] CZARNECKI, L.; Currents'Physical Components (CPC) Concept: A Fundamental of Power Theory, International School on Nonsinusoidal Currents and Compensation, p. $1-11,2008$.

[61] AKAGI, H.; KANAZAWA, Y.; NABAE, A. G.; Principles and Compensation Effectiveness of Instantaneous Reactive Power Compensator Devices, Meeting of the Power Semiconductor Converter Researchers IEE Japan, SPC 82-16, 1982. 
[62] AKAGI, H.; KANAZAWA, Y.; NABAE, A.; Generalized Theory of the Instantaneous Reactive Power in Three-Phase Circuits, International Power Electronics Conference, p. 1375-1386, 1983.

[63] TOGASAWA, S.; MUSARE, T.;NAKANO, H; NABAE, A.; Reactive Power Compensation Based on a Novel Cross Vector Theory, Transactions on Industries Applications of the IEE Japan, v. 144, n. 3, p. 340-341, 1994.

[64] NABAE, A; CAO, L.; TANAKA, T.; An Instantaneous Distortion Current Compensator without any Coordinate Transformation, International Power Electronics Conference, p. 1651-1655, 1995.

[65] AKAGI, H.; WATANABE, E.; AREDES, A.; Instantaneous Power Theory and Applications to Power Conditioning, Wiley-IEEE Press, 2007.

[66] TADMOR G.; Phasor Models in Bilinear Dissipative Systems, IEEE Conference on Dicision and Control, v. 1, p. 459-460, 2001.

[67] SANDERS, S.; NOWOROLSKI, J.; LIU, X.; VERGHESE G.; Generalized Averaging Method for Power Conversion Circuits, IEEE Transactions on Power Electronics, v. 6, p. 251-259, 1991.

[68] DE OLIVEIRA, A.; TIBURCIO, C.; LEMES, M.; RETZMANN, D.; Prospects of Voltage-Sourced Converters (VSC) Applications in DC Transmission Systems, IEEE Transmission and Distribution Conference and Exposition, p. 491-495, 2010.

[69] REED, G.; PAPE, R.; TAKEDA, M.; Advantages of Voltage Sourced Converter (VSC) Based Design Concepts for Facts and HVDC-Link Applications, IEEE Power Engineering Society General Meeting, v. 3, 2003. 
[70] ARRILlAGA, J.; LIU, Y.; WATSON, N.; Flexible Power Transmission - The HVDC Options, New York: Wiley, 2007.

[71] ACHA, E.; FUERTE-ESQUIVEL, C.; AMBRIZ-PÉREZ, H.; ANGELESCAMACHO, C.; FACTS Modeling and Simulation in Power Networks, New York: Wiley, 2004.

[72] DE LA VILLA JAEN, A.; ACHA, E.; EXPOSITO, A.; Voltage Source Converter Modeling for Power System State Estimation: STATCOM and VSC-HVDC, IEEE Transactions on Power Systems, v.23, i. 4, p. 1552-1559, 2008.

[73] YAZDANI, A.; IRAVANI, E.; Voltage Sourced Converters in Power Systems, New York: Wiley, 2010.

[74] SINGH, B.; JAYAPRAKASH, P.; KOTHARI, D.; Isolated H-bridge VSC Based 3phase 4-wire DSTATCOM for power quality improvement, IEEE International Conference on Sustainable Energy Technologies, v. 2, p. 366-371, 2008.

[75] HAITIAN WANG; GUANGFU TANG; ZHIYUAN HE; JIE YANG; Efficient Grounding for Modular Multilevel HVDC Converters (MMC) on the AC Side, IEEE Transactions on Power Delivery, v. 29, i. 3, p. 1262-1272, 2014.

[76] SOOD, V.; PATEL, H.; Comparison Between Direct And Vector Control Strategy For VSC-HVDC System In EMTP-RV, International Conference on Power India, p. 1-6, 2010. [77] STANKOVIC, A.; LIPO, T.; A Novel Control Method for Input Output Harmonic Elimination of the PWM Boost Type Rectifier under Unbalanced Operating Conditions, IEEE Transactions on Power Electronics, v.16, n.5, p.603-611, 2001. 
[78] TEODORESCU, R.; BLAABJERG, F.; LISERRE, M.; LOH, P.C.; ProportionalResonant Controllers and Filters for Grid-Connected Voltage Source Converters, IEE Proceedings Electric Power Applications, v. 153, i. 5, p. 750-762, 2006.

[79] SERPA, L.; PONNALURI, S.; BARBOSA, P.; KOLAR, J.; A Modified Direct Power Control Strategy Allowing the Connection of Three-Phase Inverters to the Grid Through LCL Filters, IEEE Transactions on Industry Applications, v. 43, n. 5, p. 1388-1400, 2007. [80] CORTES, P.; RODRIGUEZ, J.; ANTONIEWICZ, P.; KAZMIERKOWSKI, M.; Direct Power Control of an AFE Using Predictive Control, IEEE Transactions on Power Electronics, v. 23, n. 5, p. 2516-2553, 2008.

[81] MORAN, L.; ZIOGAS, P.D.; JOOS, G.; Design Aspects of Synchronous PWM Rectifier-Inverter Systems under Unbalanced Input Voltage Conditions, IEEE Transactions on Industry Applications, v. 28, i. 6, p. 1286-1293, 1992.

[82] RIOUAL, P.; POULIQUEN, H.; LOUIS, JEAN-PAUL; Regulation of a PWM Rectifier in the Unbalanced Network State Using A Generalized Model, IEEE Transactions on Power Electronics, v. 11, i. 3, p. 495-502, 1996.

[83] HOCHGRAF, C.; LASSETER, R.H.; STATCOM Controls for Operation With Unbalanced Voltages, IEEE Transactions on Power Delivery, v. 13, i. 2, p. 538-544, 1998. [84] CHEN, S.; JOOS, G.; MORAN, L.T.; Dynamic Performance of PWM STATCOM Operating under Unbalance and Fault Conditions In Distribution Systems, IEEE Power Engineering Society Winter Meeting, v. 2, p. 950-955, 2001.

[85] REYES, M.; RODRIGUEZ, P.; VAZQUEZ, S.; LUNA, A.; TEODORESCU, R.; CARRASCO, J.M.; Enhanced Decoupled Double Synchronous Reference Frame Current 
Controller for Unbalanced Grid-Voltage Conditions, IEEE Transactions on Power Electronics, v. 27, i. 9, p. 3934-3943, 2012.

[86] P. RIOUAL, H. POULIQUEN, J.P. LOUIS; Regulation of a PWM recifier in the unbalanced network state using a generalized model, IEEE Trans. Power Electron., pp. 495-502, 1996.

[87] L. XU, B. ANDERSEN, P. CARTWRIGHT; VSC transmission operating under unbalanced AC conditions - Analysis and control design, IEEE Trans. Power Deliv., 427434, 2005.

[88] H. SONG, K. NAM; Dual current control scheme for PWM converter under unbalanced input voltage conditions, IEEE Trans. Power Electron., pp. 953-959, 1999. [89] Y. ZHOU, D. JIANG, P. HU, Y. LIANG; Analysis and control of modular multilevel converters under unbalanced conditions, IEEE Trans. Power Deliv., vol. 28, no. 4, 2013. [90] A. TIMBUS, M. LISERRE, R. TEODORESCU, P. RODRIGUEZ, F. BLAABJERG; Evaluation of current controllers for distributed power generation systems, IEEE Trans. Power Electron., vol. 24, no. 3, 2009.

[91] BERGNA, G.; BERNE, E.; EGROT, P.; LEFRANC, P.; ARZANDE, A.; VANNIER, J.-C.; MOLINAS, M.; An Energy-Based Controller for HVDC Modular Multilevel Converter in Decoupled Double Synchronous Reference Frame for Voltage Oscillation Reduction, IEEE Transactions on Industrial Electronics, v. 60, i. 6, p. 2360-2371, 2013. [92] YEPES, A.G.; VIDAL, A.; LOPEZ, O.; DOVAL-GANDOY, J.; Evaluation of Techniques for Cross-Coupling Decoupling Between Orthogonal Axes in Double Synchronous Reference Frame Current Control, IEEE Transactions on Industrial Electronics, v. 61, i. 7, p. 3527-3531, 2014. 
[93] CIOBOTARU, M.; TEODORESCU, R.; BLAABJERG, F.; A New Single-Phase PLL Structure Based on Second Order Generalized Integrator, IEEE Power Electronics Specialists Conference, p. 1-6, 2006.

[94] RODRIGUEZ, P.; LUNA, A.; CANDELA, I.; MUJAL, R.; TEODORESCU, R.; BLAABJERG, F.; Multiresonant Frequency-Locked Loop for Grid Synchronization of Power Converters under Distorted Grid Conditions, IEEE Transactions on Industrial Electronics, v. 58, i. 1, p. 127-138, 2011.

[95] DURRANT, M.; WERNER, H.; ABBOTT, K.; Model of a VSC HVDC Terminal Attached to a Weak AC System, IEEE Conference on Control Applications, v. 1, p. 178$182,2003$.

[96] JOVCIC, D.; LAMONT, L.; XU, L.; VSC Transmission Model for Analytical Studies, IEEE Power Engineering Society General Meeting, v. 3, 2003. 